\title{
Cinchona Alkaloids Squaramide Catalyzed Asymmetric Ugi-Type Reaction of Isocyanoacetates with C,N-Cyclic Azomethine Imines: Access to Chiral Oxazole-Substituted Tetrahydroisoquinolines
}

\author{
Zi-Qiang Zhao, ${ }^{\text {a }}$ Xiao-Li Zhao, ${ }^{\mathrm{b}}$ Min Shi, ${ }^{\text {a.c }}$ and Mei-Xin Zhao, ${ }^{\text {a,* }}$ \\ ${ }^{a}$ Key Laboratory for Advanced Materials and Institute of Fine Chemicals, School of Chemistry \& Molecular \\ Engineering, East China University of Science and Technology, 130 Mei Long Road, Shanghai, 200237, P. R. China \\ E-mail:mxzhao@ecust.edu.cn \\ b Department of Chemistry, East China Normal University, 3663 N. Zhongshan Road, Shanghai, 200062, China. \\ c State Key Laboratory of Organometallic Chemistry, Shanghai Institute of Organic Chemistry, Chinese Academy of \\ Sciences, 354 Fenglin Road, Shanghai 200032, P. R. China.
}

\section{Table of Contents}

1. Optimization reaction conditions of catalytic asymmetric formal [3+3] cycloaddition of isocyanoacetate 2a to $\mathrm{C}, \mathrm{N}$-cyclic azomethine imine $\mathbf{5 a}$

2. X-Ray crystal data of compound $\mathbf{8 j}$

3. Copies of HPLC analysis spectra of compounds $7 \mathbf{a}$ and $\mathbf{8}$

4. Copies of NMR spectra for the compounds $7 \mathbf{a}$ and $\mathbf{8}$ 


\section{Optimization reaction conditions of catalytic asymmetric formal $[3+3]$ cycloaddition of}

isocyanoacetate 2a to $\mathrm{C}, \mathrm{N}$-cyclic azomethine imine $5 \mathrm{a}$

Table S1. Catalysts screening
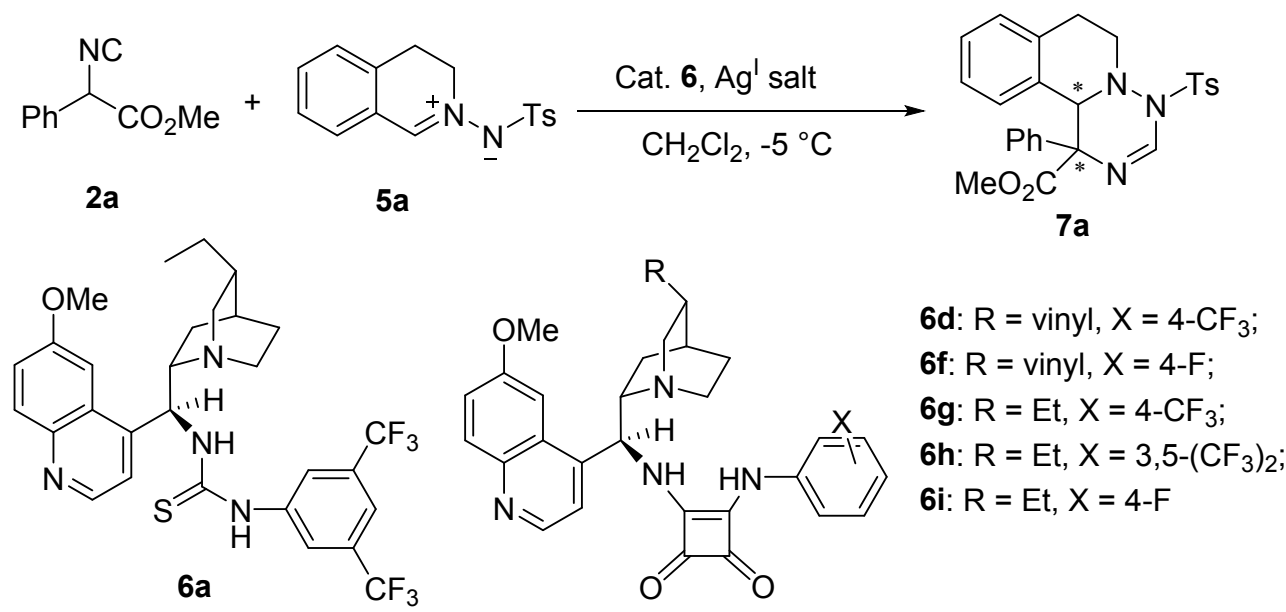

\begin{tabular}{ccccccc}
\hline Entry & Cat. $\mathbf{6}$ & $\mathrm{Ag}(\mathrm{I}) \mathrm{salt}$ & $t(\mathrm{~h})$ & yield $(\%)^{\mathrm{b}}$ & $e e(\%)^{\mathrm{c}}$ & $d r^{\mathrm{d}}$ \\
\hline 1 & $\mathbf{6 a}$ & $\mathrm{AgNO}_{3}$ & 41 & 41 & 24 & $>20: 1$ \\
2 & $\mathbf{6 d}$ & $\mathrm{AgNO}_{3}$ & 47 & 51 & 50 & $>20: 1$ \\
3 & $\mathbf{6 f}$ & $\mathrm{AgNO}_{3}$ & 47 & 52 & 47 & $>20: 1$ \\
4 & $\mathbf{6 g}$ & $\mathrm{AgNO}_{3}$ & 40 & 55 & 52 & $>20: 1$ \\
5 & $\mathbf{6 h}$ & $\mathrm{AgNO}_{3}$ & 37 & 52 & 32 & $>20: 1$ \\
6 & $\mathbf{6 i}$ & $\mathrm{AgNO}_{3}$ & 40 & 52 & 48 & $>20: 1$ \\
7 & $\mathbf{6 g}$ & $\mathrm{Ag}_{2} \mathrm{O}$ & 27 & 42 & 47 & $>20: 1$ \\
8 & $\mathbf{6 g}$ & $\mathrm{Ag}_{2} \mathrm{CO}_{3}$ & 23 & 41 & 44 & $>20: 1$ \\
9 & $\mathbf{6 g}$ & $\mathrm{AgOTf}$ & 36 & 43 & 46 & $>20: 1$ \\
10 & $\mathbf{6 g}$ & $\mathrm{AgSbF}_{6}$ & 39 & 52 & 45 & $>20: 1$ \\
11 & $\mathbf{6 g}$ & $\mathrm{AgOAc}$ & 27 & 42 & 47 & $>20: 1$ \\
\hline
\end{tabular}

a. All reactions were carried out with $0.10 \mathrm{mmol}$ of isocyanoacetate $\mathbf{2 a}, 0.12 \mathrm{mmol}$ of $\mathrm{C}, \mathrm{N}$-cyclic azomethine imines 5a, cat. $6(20 \mathrm{~mol} \%), \operatorname{Ag}(\mathrm{I})$ salt $(10 \mathrm{~mol} \%)$ in $1.0 \mathrm{~mL}$ of DCM at $-5{ }^{\circ} \mathrm{C}$. ${ }^{b}$. Yield of isolated product. ${ }^{c}$ Determined by HPLC analysis on a chiral stationary phase. ${ }^{d}$. Determined by ${ }^{1} \mathrm{H}$ NMR analysis for the pure product. 
Table S2. Optimization of Reaction Conditions ${ }^{a}$

\begin{tabular}{ccccccc}
\hline Entry & Solvent & $\mathrm{T}\left({ }^{\circ} \mathrm{C}\right)$ & $t(\mathrm{~h})$ & yield $(\%)^{\mathrm{b}}$ & $e e(\%)^{\mathrm{c}}$ & $d r^{\mathrm{d}}$ \\
\hline 1 & $\mathrm{DCM}$ & -10 & 40 & 55 & 58 & $>20: 1$ \\
2 & $\mathrm{DCM}$ & -20 & 44 & 52 & 52 & $>20: 1$ \\
3 & $\mathrm{DCM}$ & r.t. & 24 & 43 & 40 & $>20: 1$ \\
4 & $\mathrm{CHCl}_{3}$ & -10 & 40 & 55 & 55 & $>20: 1$ \\
5 & $\mathrm{DCE}$ & -10 & 40 & 54 & 57 & $>20: 1$ \\
6 & $\mathrm{Et}{ }_{2} \mathrm{O}$ & -10 & 38 & 49 & 45 & $>20: 1$ \\
7 & $\mathrm{THF}$ & -10 & 39 & 51 & 49 & $>20: 1$ \\
8 & $\mathrm{MeOH}$ & -10 & 35 & 40 & 41 & $>20: 1$ \\
9 & $\mathrm{EA}$ & -10 & 41 & 52 & 51 & $>20: 1$ \\
10 & $\mathrm{CH}_{3} \mathrm{CN}$ & -10 & 37 & 41 & 39 & $>20: 1$ \\
$11^{\mathrm{e}}$ & $\mathrm{DCM}$ & -10 & 45 & 60 & 61 & $>20: 1$ \\
$12^{\mathrm{f}}$ & $\mathrm{DCM}$ & -10 & 46 & 47 & 52 & $>20: 1$ \\
\hline
\end{tabular}

${ }^{a}$ Unless otherwise noted, all reactions were carried out with $0.10 \mathrm{mmol}$ of isocyanoacetate $\mathbf{2 a}, 0.12 \mathrm{mmol}$ of C,N-cyclic azomethine imines 5a, cat. $\mathbf{6 g}(20 \mathrm{~mol} \%), \mathrm{AgNO}_{3}(10 \mathrm{~mol} \%)$ in $1.0 \mathrm{~mL}$ of solvent. ${ }^{b}$ Yield of isolated product. ${ }^{c}$ Determined by HPLC analysis on a chiral stationary phase. ${ }^{d}$ Determined by ${ }^{1} \mathrm{H}$ NMR analysis. ${ }^{e}$ Using $20 \mathrm{mo} \%$ of cat. $6 \mathrm{~g}$ and $5 \mathrm{~mol} \% \mathrm{AgNO}_{3} .{ }^{f}$ Using $10 \mathrm{mo} \%$ of cat. $6 \mathbf{g}$ and $5 \mathrm{~mol} \% \mathrm{AgNO}_{3}$.

Methyl 1-phenyl-4-tosyl-1,4,6,7-tetrahydro-11bl3-[1,2,4]triazino[6,1-a]isoquinoline-1-carboxylate (7a) (Table S2, entry 11). White solid; yield $28.5 \mathrm{mg}(60 \%)$; mp 166.9-168.0 ${ }^{\circ} \mathrm{C} .[\alpha]_{\mathrm{D}}^{20}-221.4\left(c 1.00, \mathrm{CH}_{2} \mathrm{Cl}_{2}\right)(61 \%$ ee); the $e e$ was determined by HPLC analysis with a Chiralpak AD-H column $(75 / 25$ hexane $/ i-\mathrm{PrOH} ; 0.8 \mathrm{~mL} / \mathrm{min} ; \lambda=254$ $\left.\mathrm{nm} ; t_{\text {major }}=9.47 \mathrm{~min} ; t_{\text {minor }}=13.94 \mathrm{~min}\right) ;{ }^{1} \mathrm{H} \mathrm{NMR}\left(\mathrm{CDCl}_{3}, 400 \mathrm{MHz}\right) \delta 8.55(\mathrm{~s}, 1 \mathrm{H}), 7.81(\mathrm{~d}, J=8.4 \mathrm{~Hz}, 2 \mathrm{H})$, 7.28-7.26 (m, 5H), $7.12(\mathrm{q}, J=7.6 \mathrm{~Hz}, 2 \mathrm{H}), 6.91-6.88(\mathrm{~m}, 2 \mathrm{H}), 6.68(\mathrm{t}, J=6.4 \mathrm{~Hz}, 1 \mathrm{H}), 5.34(\mathrm{~d}, J=8.0 \mathrm{~Hz}, 1 \mathrm{H})$, $3.63(\mathrm{~s}, 3 \mathrm{H}), 3.48-3.41(\mathrm{~m}, 1 \mathrm{H}), 3.32-3.17(\mathrm{~m}, 2 \mathrm{H}), 3.03(\mathrm{~s}, 1 \mathrm{H}), 2.91-2.86(\mathrm{~m}, 1 \mathrm{H}), 2.39(\mathrm{~s}, 3 \mathrm{H}) ;{ }^{13} \mathrm{C}\left\{{ }^{1} \mathrm{H}\right\} \mathrm{NMR}$ $\left(\mathrm{CDCl}_{3}, 100 \mathrm{MHz}\right) \delta 169.3,145.3,140.7,138.8,133.5,133.0,130.7,129.7,128.1,128.1,127.9,127.8,127.5$, 127.3, 127.2, 123.7, 64.4, 64.2, 52.7, 48.2, 28.1, 21.6; IR (Film) v 1733, 1633, 1598, 1489, 1451, 1436, 1368, 1351, $1242 \mathrm{~cm}^{-1}$; HRMS (ESI-TOF) m/z: [M+H] $]^{+}$calcd for $\mathrm{C}_{26} \mathrm{H}_{26} \mathrm{~N}_{3} \mathrm{O}_{4} \mathrm{~S}$ 476.1644; Found 476.1636. 


\section{X-Ray Crystal Data of Compound 8j}

The single crystal of compound $\mathbf{8 j}$ was obtained by recrystallization from $\mathrm{CHCl}_{3} / \mathrm{n}$-hexane (1:1) at room temperature. The ellipsoid contour was set at $50 \%$ probability levels. The crystal data of compound $\mathbf{8 j}$ have been deposited in CCDC with number 1904277.

Table S3. Crystal data and structure refinement for 8j (CCDC 1904277).

Empirical formula

Formula weight

Temperature

Wavelength

Crystal system

Space group

Unit cell dimensions

Volume

Z

Density (calculated)

Absorption coefficient

$\mathrm{F}(000)$

Crystal size

Theta range for data collection

Index ranges

Reflections collected

Independent reflections

Data / restraints / parameters

Goodness-of-fit on $\mathrm{F}^{2}$

Final $\mathrm{R}$ indices $[\mathrm{I}>2 \operatorname{sigma}(\mathrm{I})]$

$\mathrm{R}$ indices (all data)

Largest diff. peak and hole
$\mathrm{C}_{26} \mathrm{H}_{24} \mathrm{BrN}_{3} \mathrm{O}_{4} \mathrm{~S}$

554.45

293(2)

$1.54184 \AA$

monoclinic

P 1211

$\mathrm{a}=11.8479(2) \AA$

$\alpha=90^{\circ}$.

$\mathrm{b}=10.01910(10) \AA$

$\beta=116.482(2)^{\circ}$.

$\mathrm{c}=11.9095(2) \AA$

$\gamma=90^{\circ}$.

$1265.38(4) \AA^{3}$

2

$1.455 \mathrm{Mg} / \mathrm{m}^{3}$

$3.297 \mathrm{~mm}^{-1}$

568

$0.42 \times 0.36 \times 0.35 \mathrm{~mm}^{3}$

4.1420 to $74.0850^{\circ}$.

$-14<=\mathrm{h}<=14,-11<=\mathrm{k}<=11,-14<=\mathrm{l}<=14$

28598

$4806[R($ int $)=0.1046]$

$4806 / 1 / 318$

1.054

$\mathrm{R} 1=0.0420, \mathrm{wR} 2=0.1116$

$\mathrm{R} 1=0.0436, \mathrm{wR} 2=0.1106$

0.578 and -0.651 e. $\AA^{-3}$ 


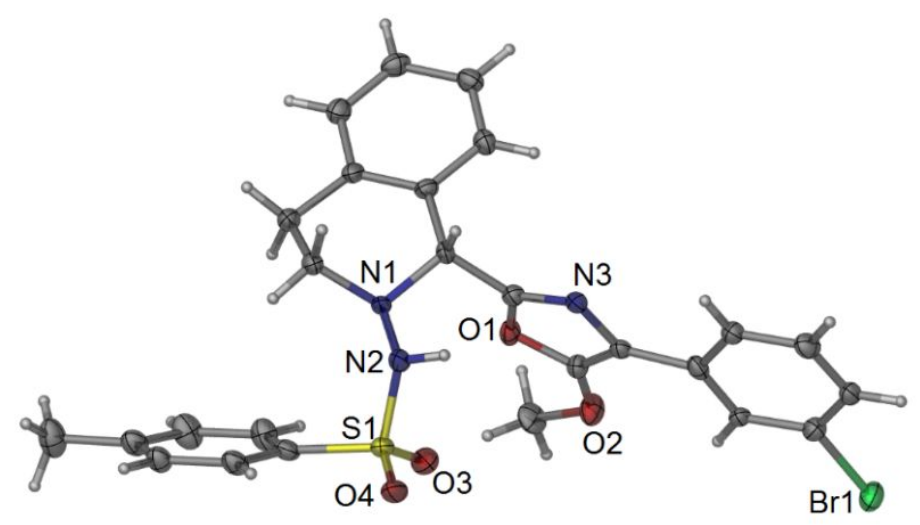

Figure S1. ORTEP plot of the X-ray crystal structure of $\mathbf{8 j}$. Displacement ellipsoids are drawn at the $50 \%$ probability level. 


\section{Copies of HPLC Analysis Spectra of Compounds 7a and 8}

\section{7a (Table S2, entry 11)}<smiles>[Y5]C12C(C(=O)OC)=NC=NN1CCc1ccccc12</smiles>

\section{Racemic}

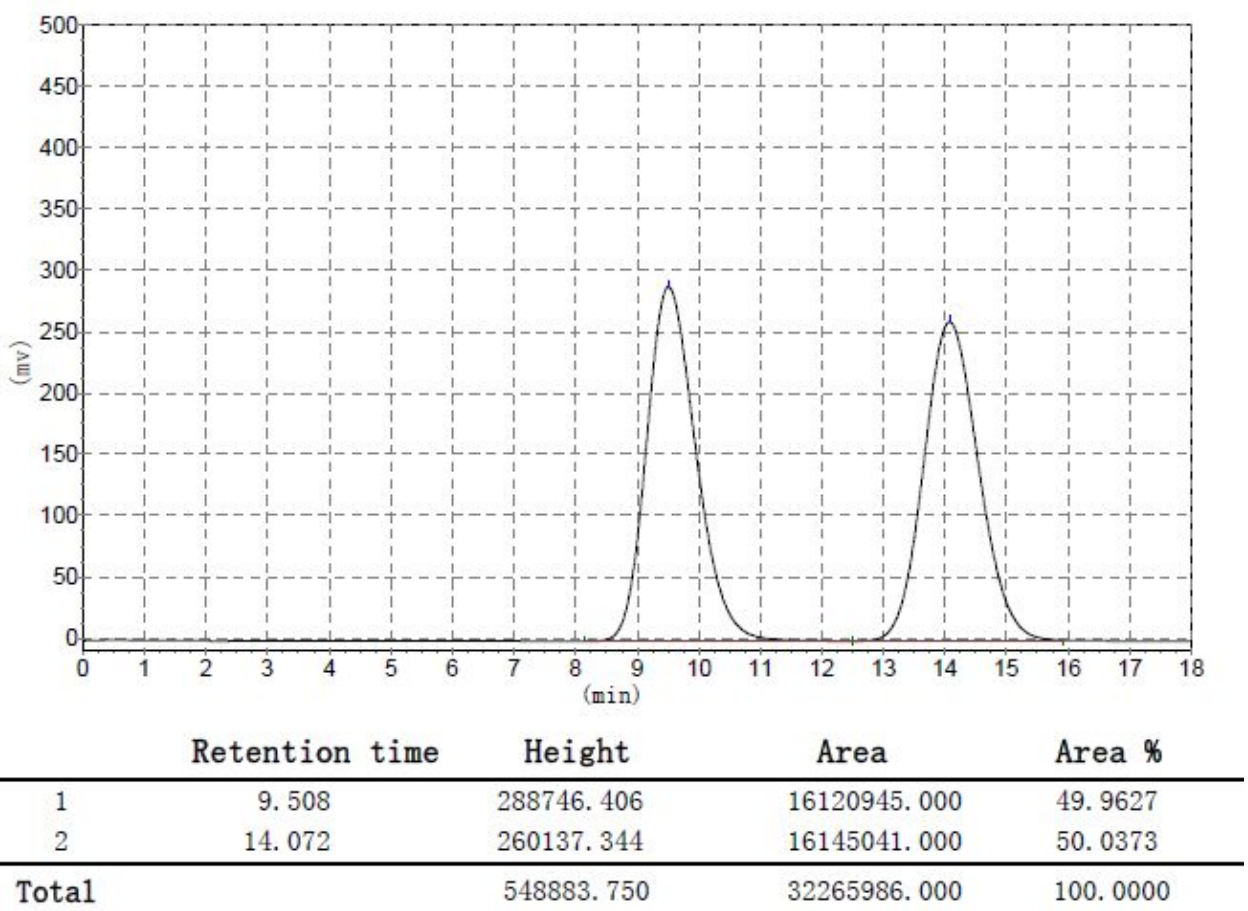

\section{Chiral}

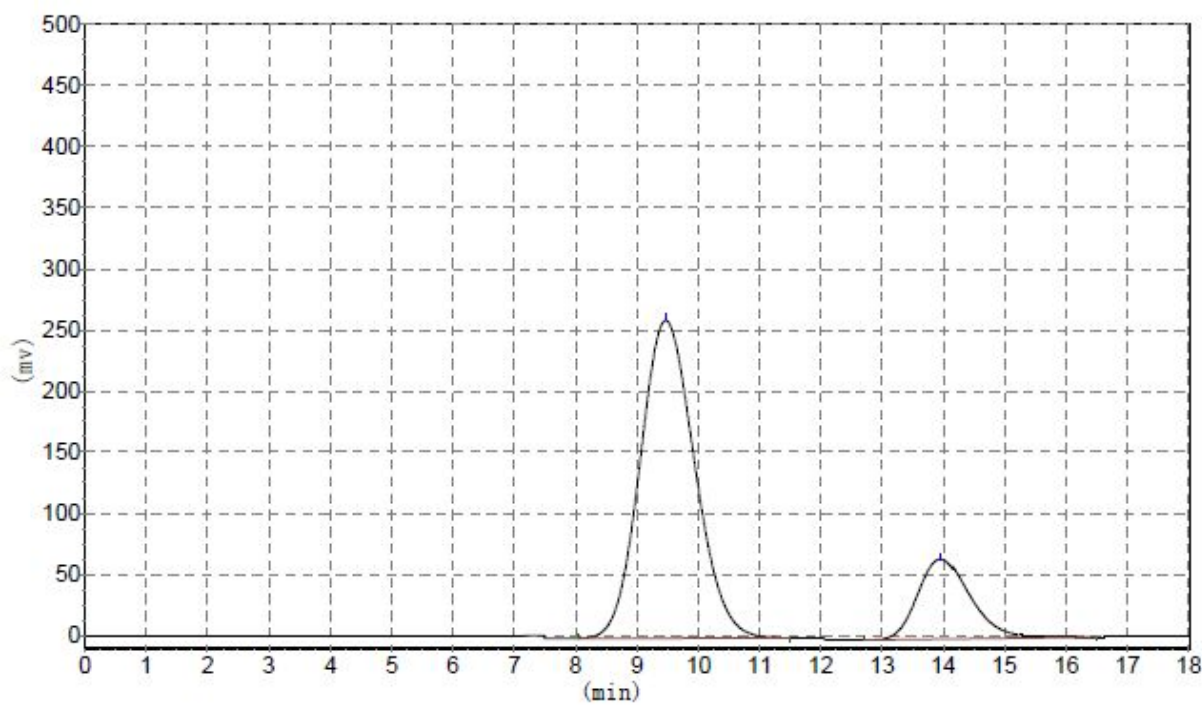

\begin{tabular}{ccccc} 
& Retention time & Height & Area & Area\% \\
\hline 1 & 9.468 & 259739.172 & 16062318.000 & 80.4395 \\
2 & 13.945 & 65206.301 & 3905867.500 & 19.5605 \\
\hline Total & & 324945.473 & 19968185.500 & 100.0000
\end{tabular}




\section{8a (Table 3, entry 1)}<smiles></smiles>

\section{Racemic}

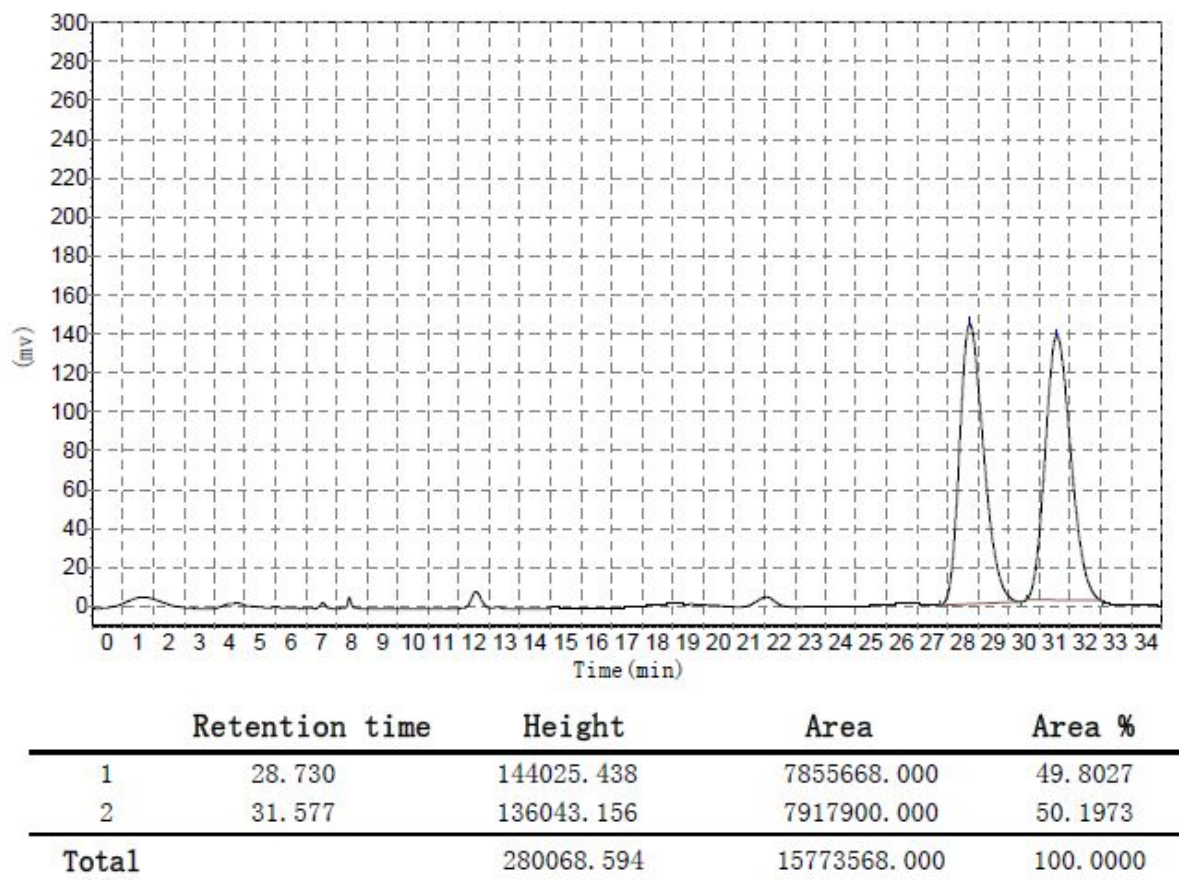

\section{Chiral}

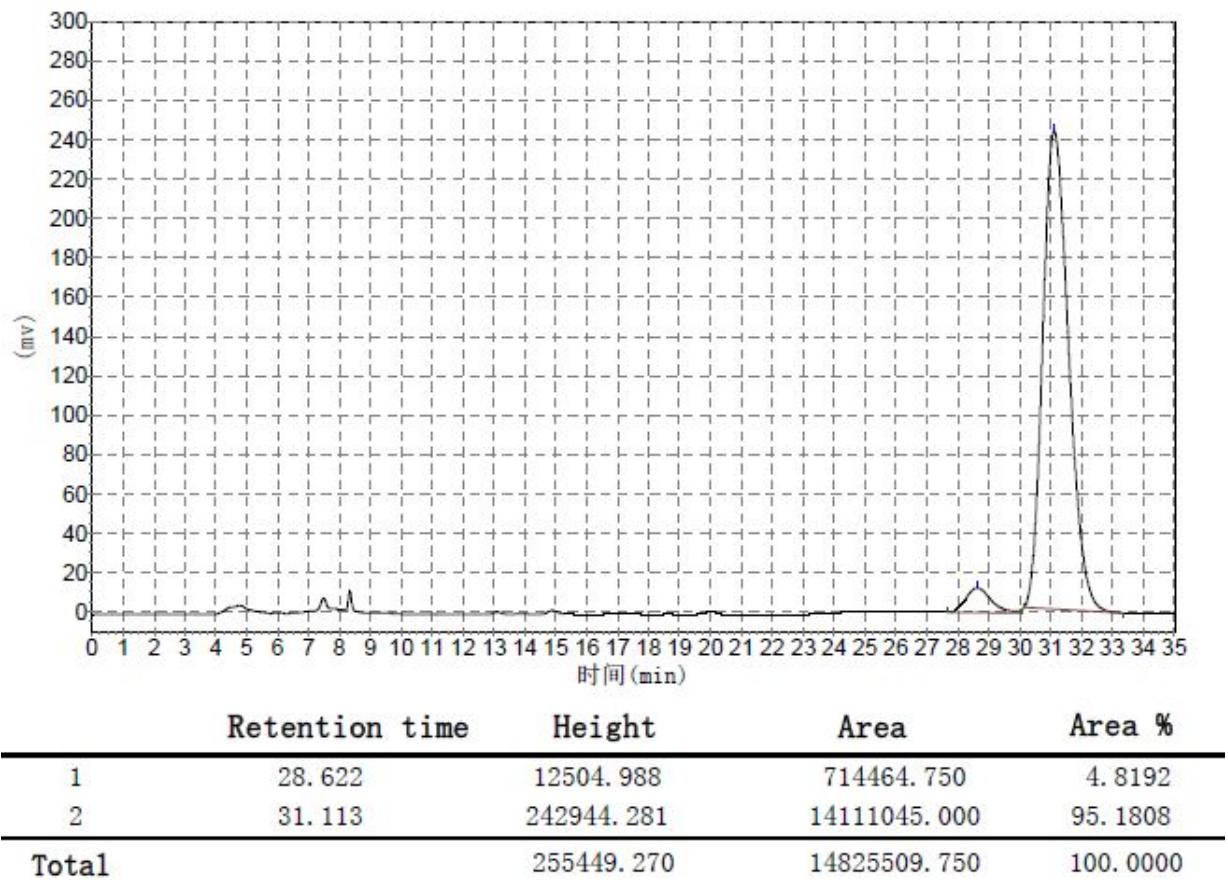




\section{$8 b$ (Table 3, entry 2)}<smiles>COc1oc([C@@H]2c3ccccc3CCN2N[In])nc1-c1ccc(F)cc1</smiles>

\section{Racemic}

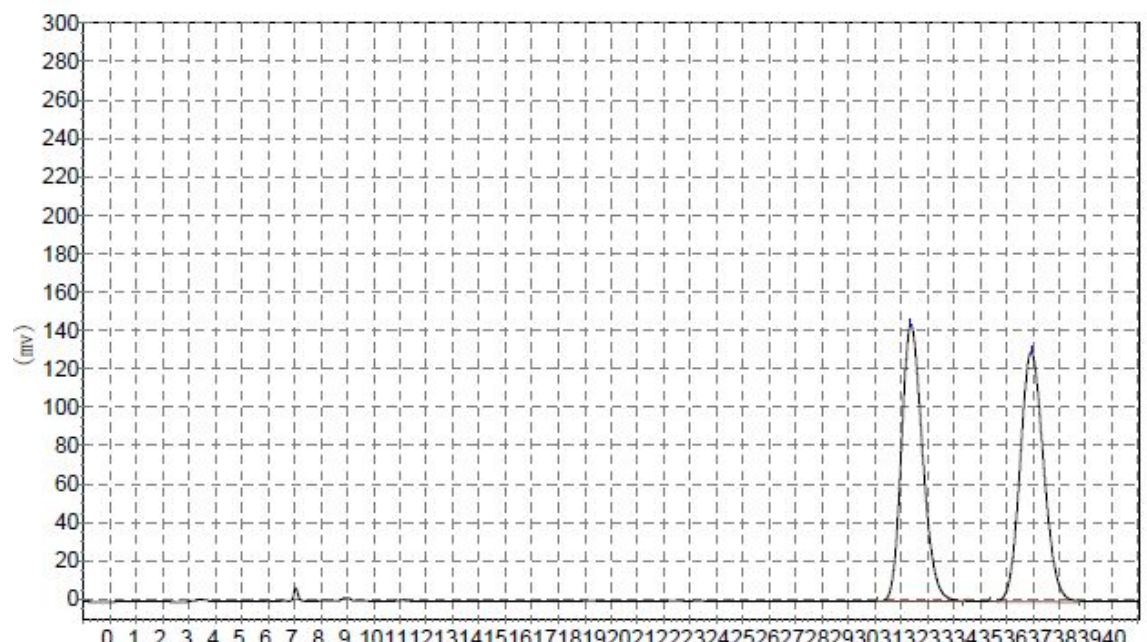

(9in)

\begin{tabular}{ccccc} 
& Retention time & Height & Area & Area \% \\
\hline 1 & 31.375 & 144372.156 & 8048178.500 & 49.8721 \\
2 & 35.938 & 130068.156 & 8072773.500 & 50.0245 \\
\hline Tota1 & & 274821.476 & 16137641.910 & 100.0000
\end{tabular}

\section{Chiral}

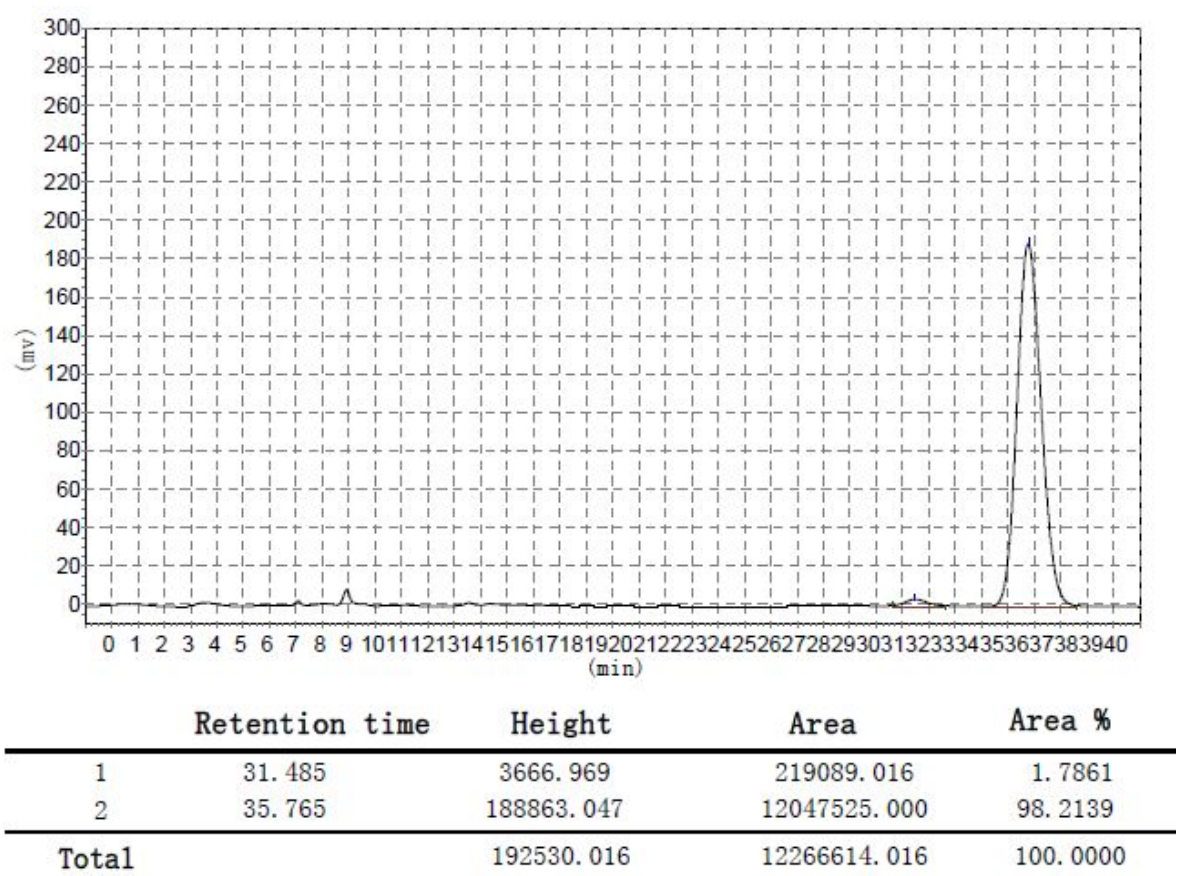




\section{8c (Table 3, entry 3)}<smiles>COc1oc([C@@H]2c3ccccc3CCN2N[I+])nc1-c1ccc(Cl)cc1</smiles>

\section{Racemic}

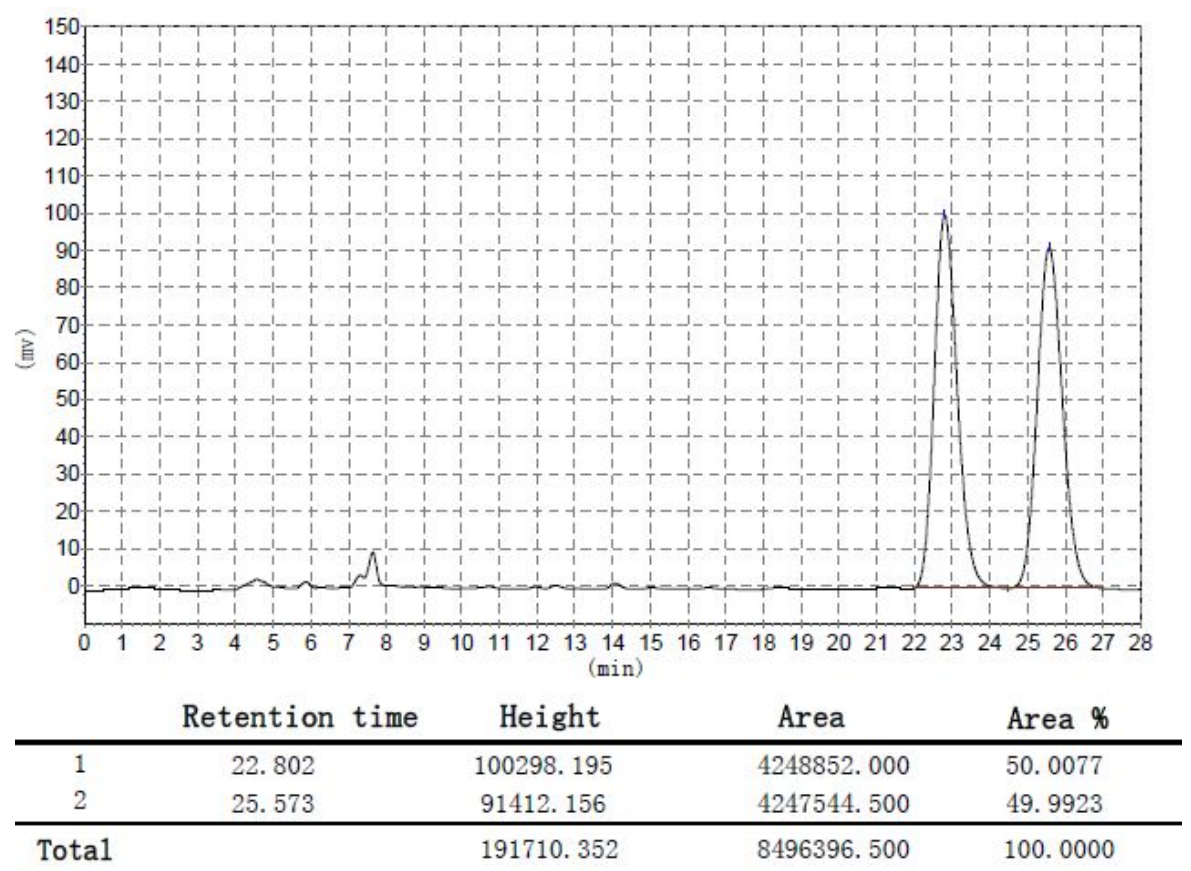

\section{Chiral}

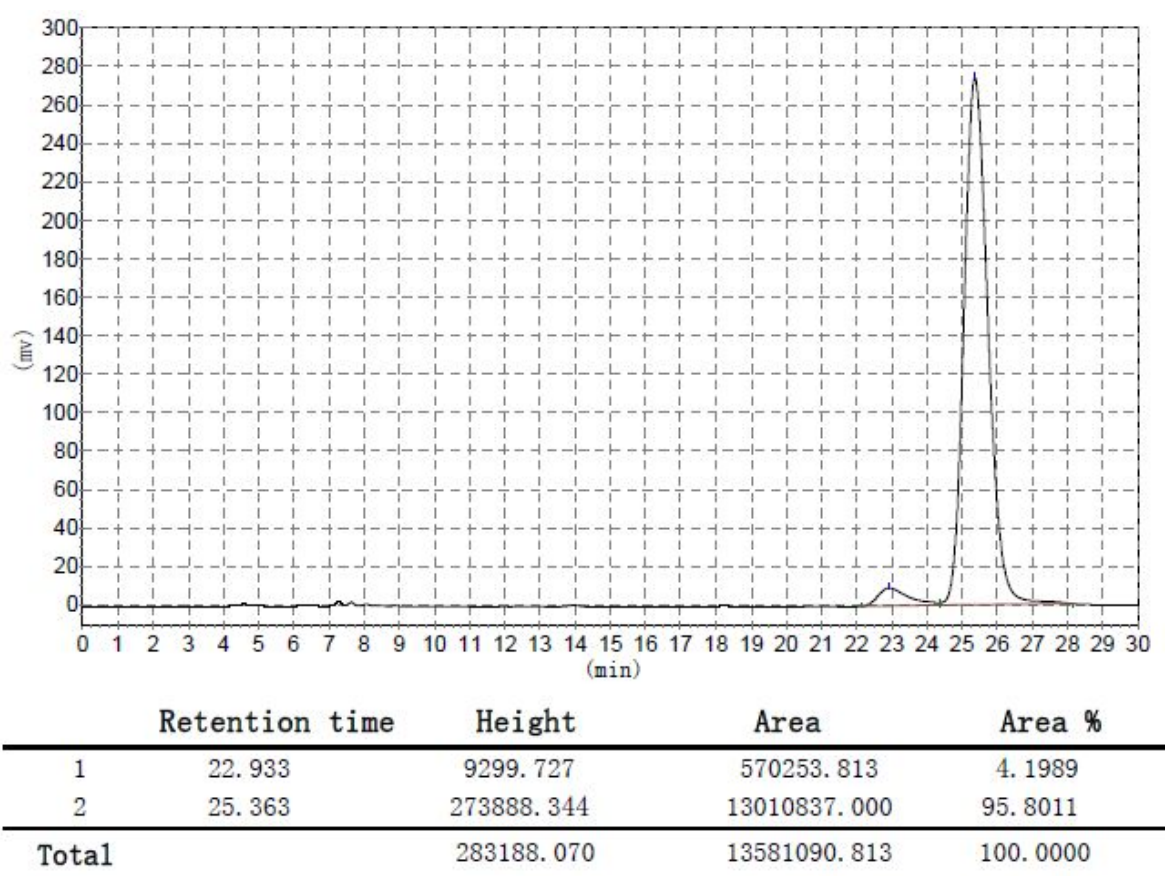




\section{8d (Table 3, entry 4)}<smiles>COc1oc([C@@H]2c3ccccc3CCN2N[I+])nc1-c1ccc(Br)cc1</smiles>

\section{Racemic}

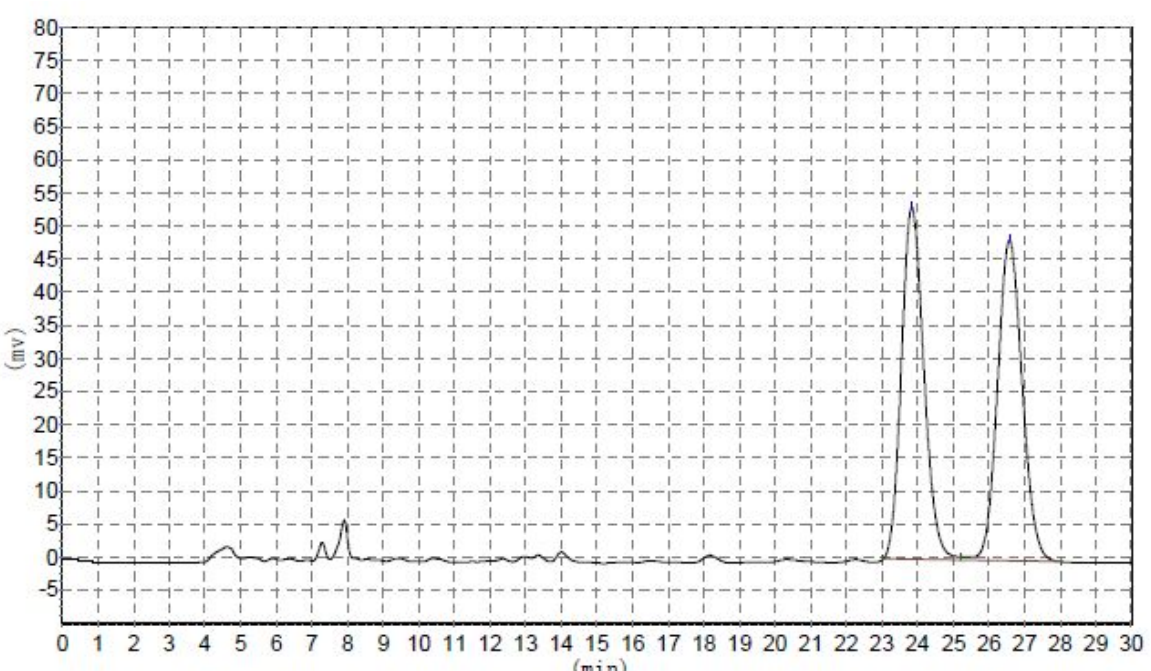

\begin{tabular}{ccccc} 
& Retention time & Height & Area & Area $\%$ \\
\hline 1 & 23.837 & 53173.168 & 2327695.750 & 49.9124 \\
2 & 26.577 & 48402.313 & 2335866.750 & 50.0876 \\
\hline Total & & 101575.480 & 4663562.500 & 100.0000
\end{tabular}

\section{Chiral}

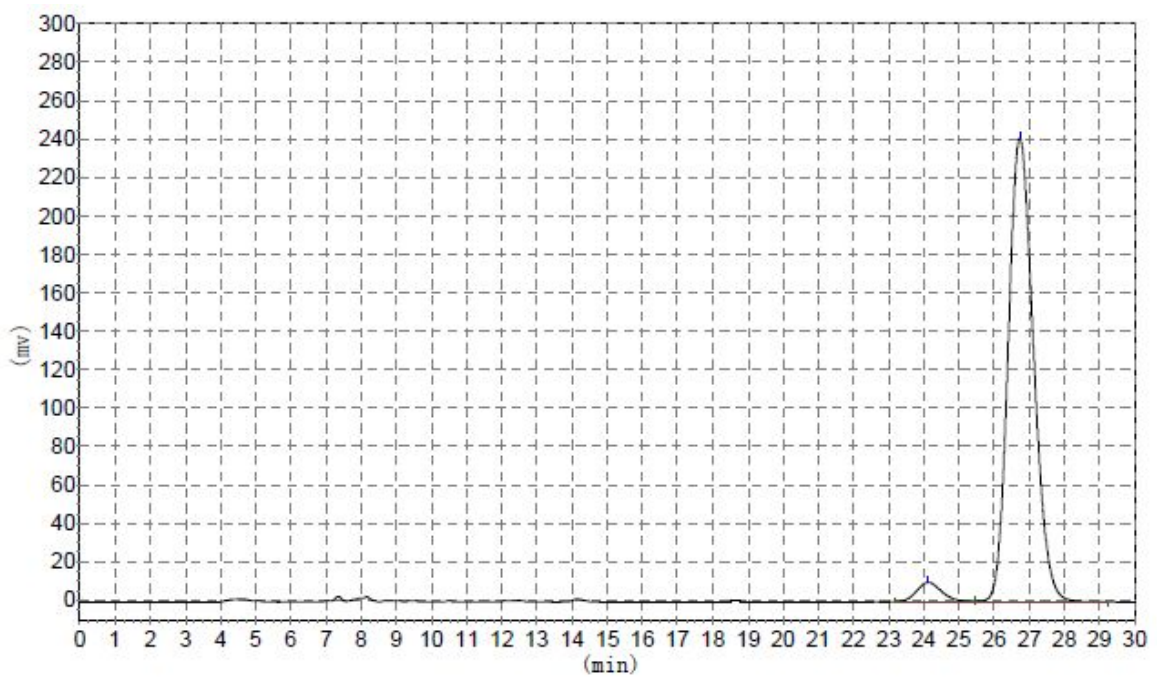

\begin{tabular}{ccccc} 
& Retention time & Height & Area & Area $\%$ \\
\hline 1 & 24.125 & 10020.110 & 502774.594 & 4.0178 \\
2 & 26.732 & 241427.406 & 12010808.000 & 95.9822 \\
\hline Tota1 & & 251447.517 & 12513582.594 & 100.0000
\end{tabular}




\section{8e (Table 3, entry 5)}<smiles>COc1oc([C@@H]2c3ccccc3CCN2N[I+])nc1-c1ccc(C(F)(F)F)cc1</smiles>

\section{Racemic}

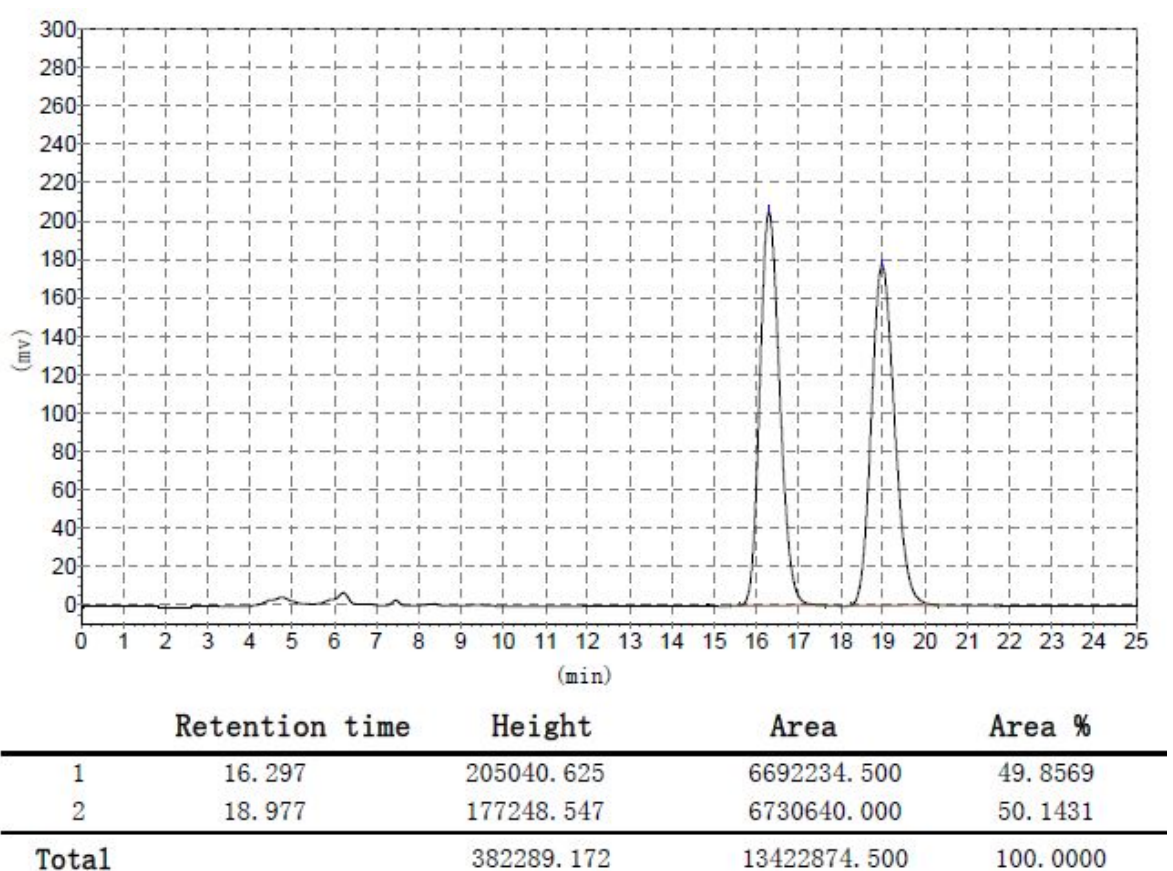

\section{Chiral}

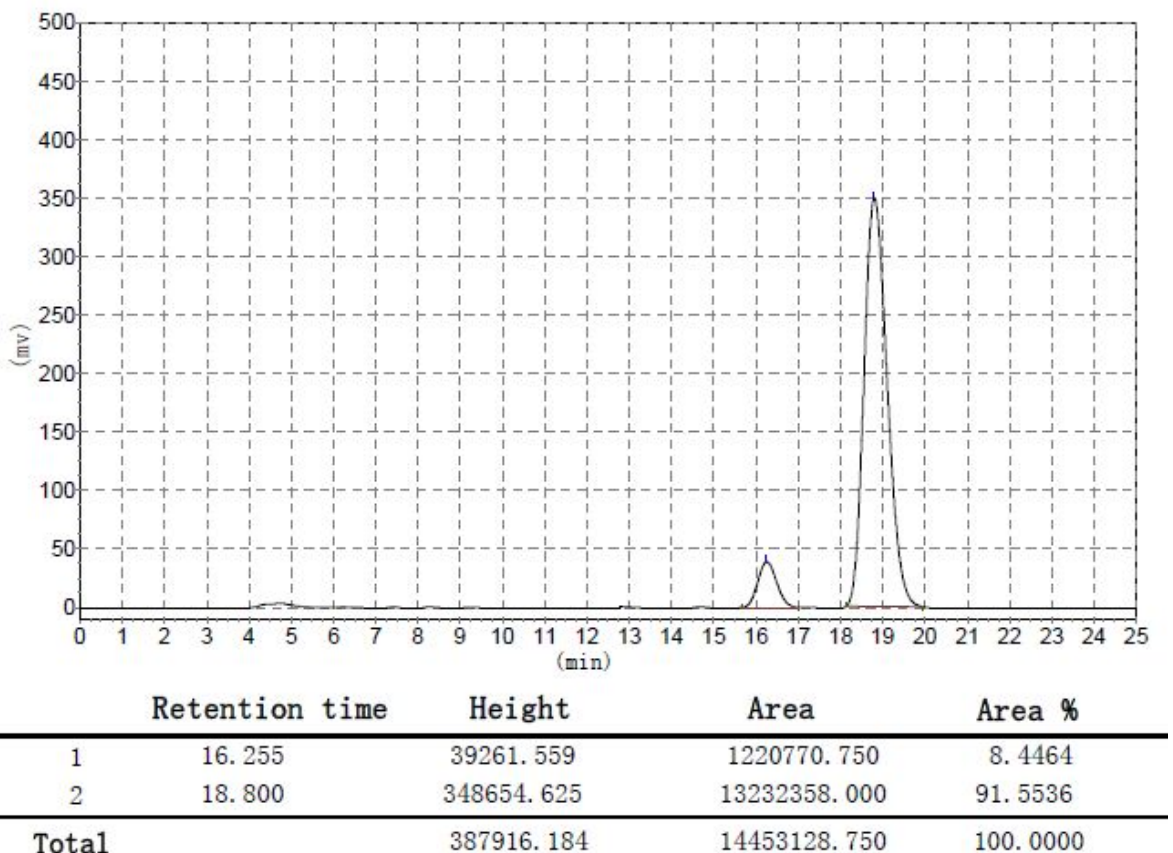




\section{8f (Table 3, entry 6)}<smiles>COc1oc([C@@H]2c3ccccc3CCN2N[I+])nc1-c1ccc(C)cc1</smiles>

\section{Racemic}

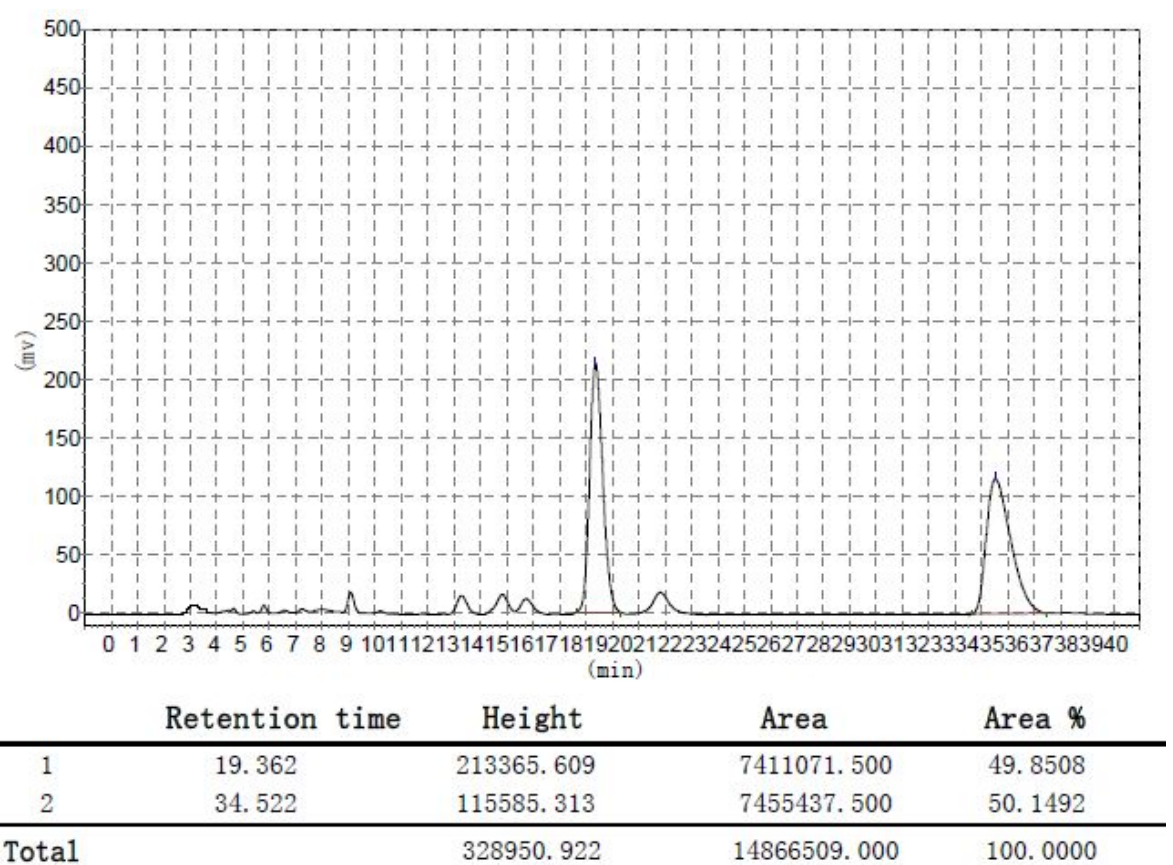

\section{Chiral}

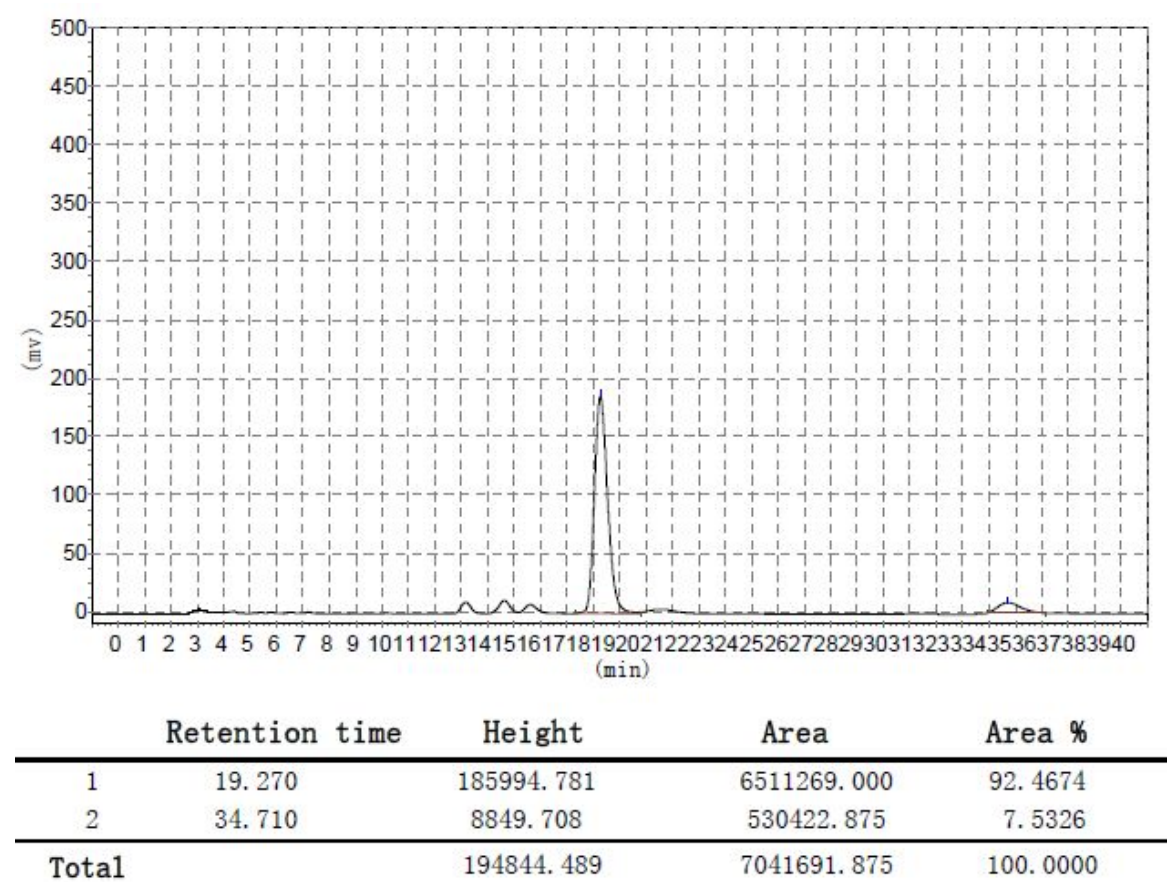




\section{$8 g$ (Table 3, entry 7)}

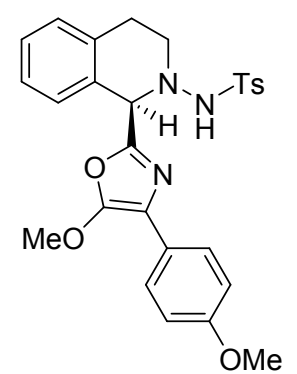

\section{Racemic}

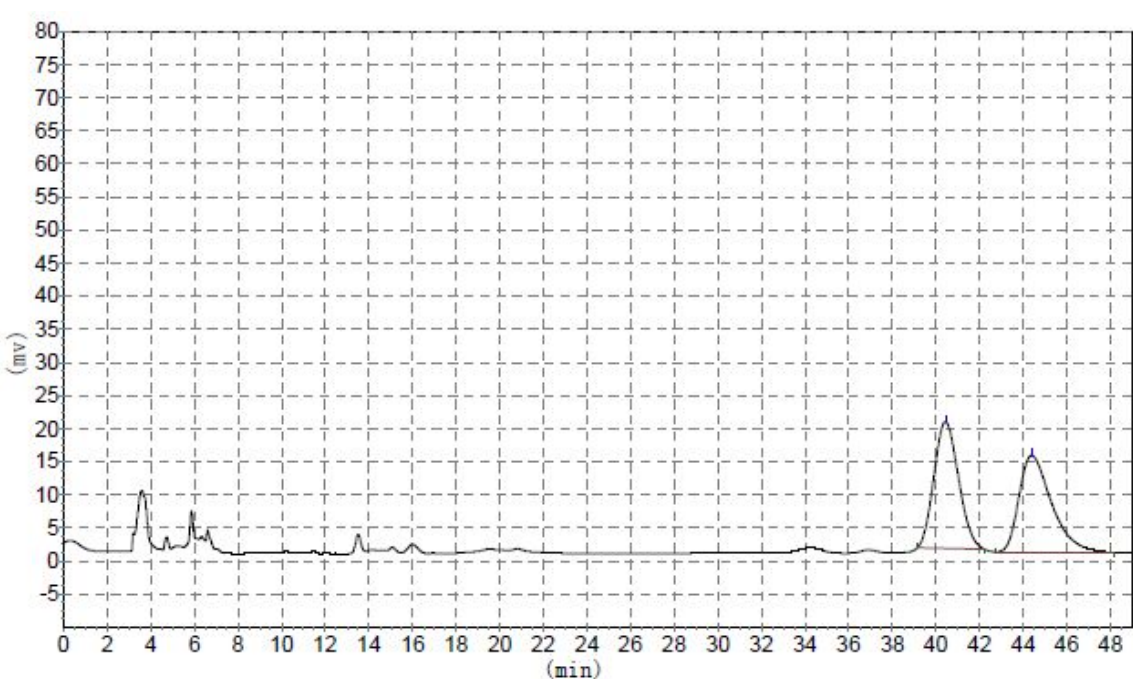

\begin{tabular}{ccccc} 
& Retention time & Height & Area & Area \% \\
\hline 1 & 40.428 & 19143.639 & 1497917.750 & 49.8096 \\
2 & 44.388 & 14602.064 & 1509366.625 & 50.1903 \\
\hline Tota1 & & 33745.703 & 3007284.375 & 100.0000
\end{tabular}

\section{Chiral}

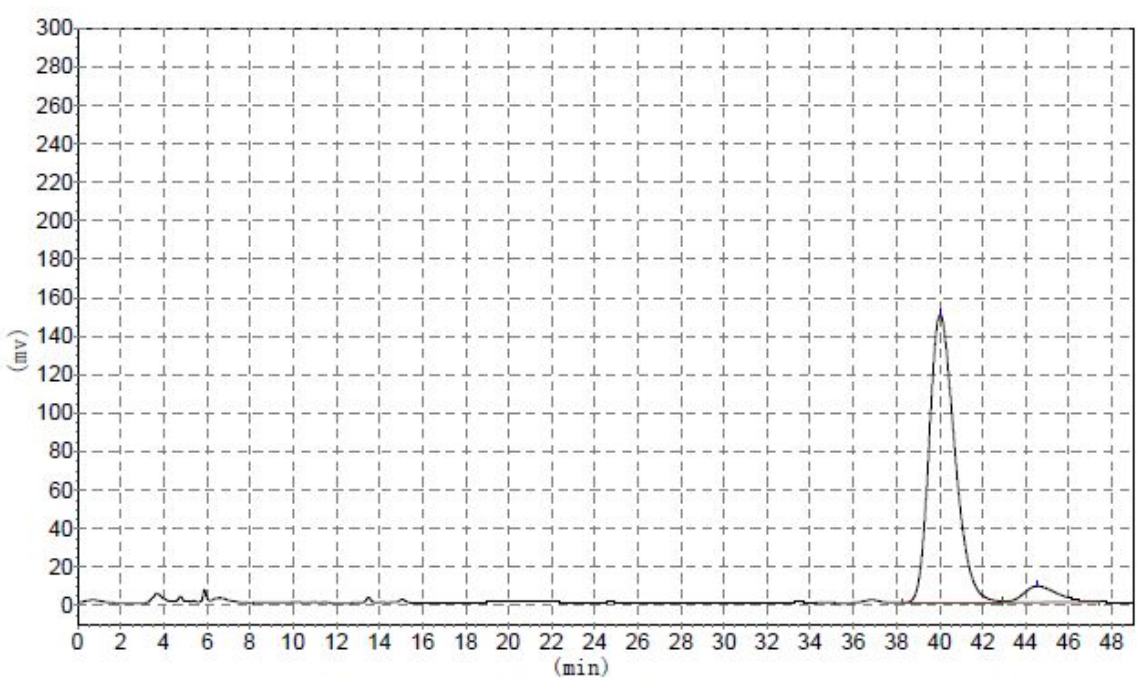

\begin{tabular}{ccccc} 
& Retention time & Height & Area & Area \% \\
\hline 1 & 40.033 & 149729.969 & 12330603.000 & 93.1064 \\
2 & 44.560 & 8373.181 & 912954.438 & 6.8936 \\
\hline Total & & 158103.149 & 13243557.438 & 100.0000
\end{tabular}




\section{8h (Table 3, entry 8)}

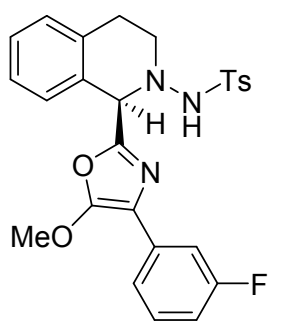

\section{Racemic}

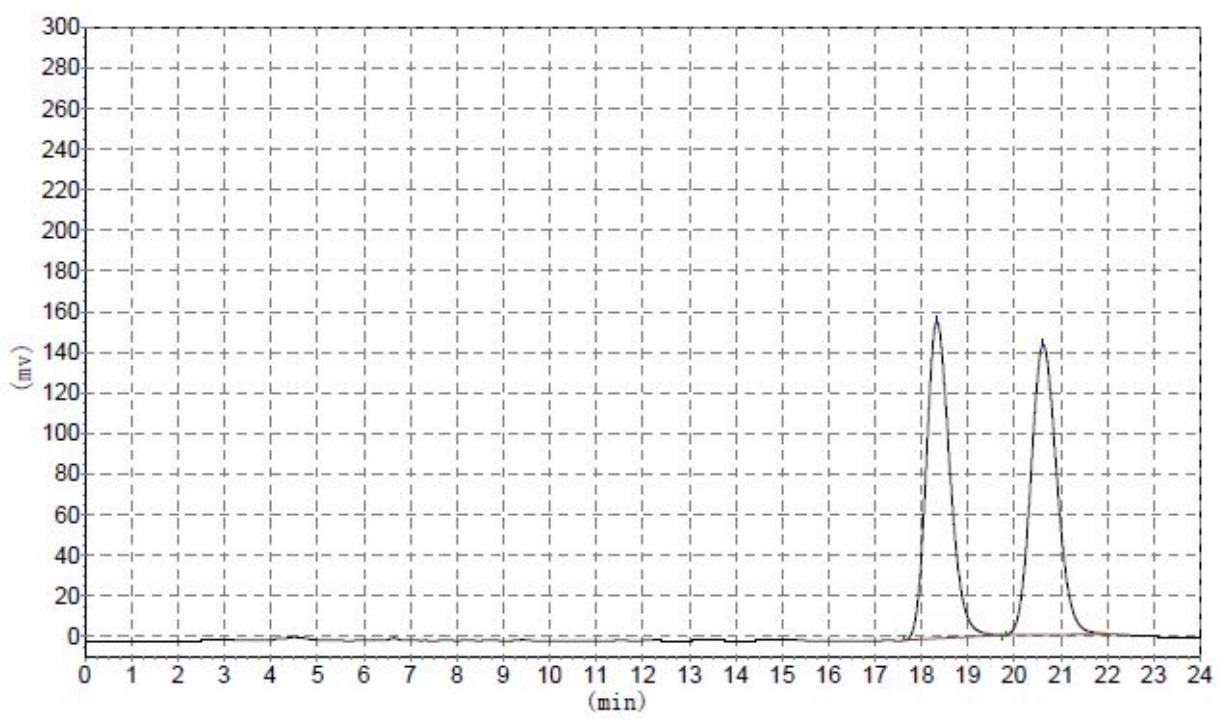

\begin{tabular}{ccccc} 
& Retention time & Height & Area & Area \% \\
\hline 1 & 18.335 & 156049.313 & 5512683.000 & 49.9944 \\
2 & 20.623 & 142782.953 & 5513916.000 & 50.0056 \\
\hline Tota1 & & 298832.266 & 11026599.000 & 100.0000
\end{tabular}

Chiral

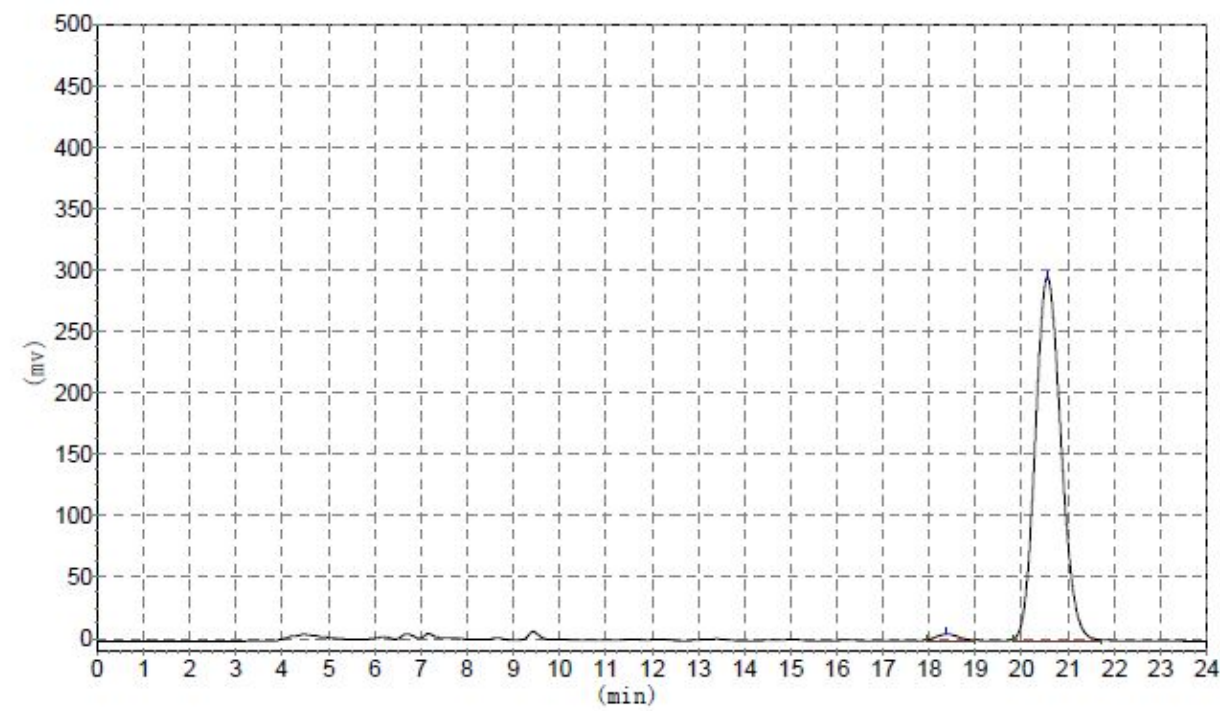

\begin{tabular}{ccccc} 
& Retention time & Height & Area & Area \% \\
\hline 1 & 18.370 & 5578.047 & 190449.156 & 1.6377 \\
2 & 20.550 & 296244.313 & 11438650.000 & 98.3623 \\
\hline Total & & 301822.359 & 11629099.156 & 100.0000
\end{tabular}




\section{8i (Table 3, entry 9)}<smiles>[3H]NN1CCc2ccccc2[C@H]1c1nc(-c2cccc(Cl)c2)c(OC)o1</smiles>

\section{Racemic}

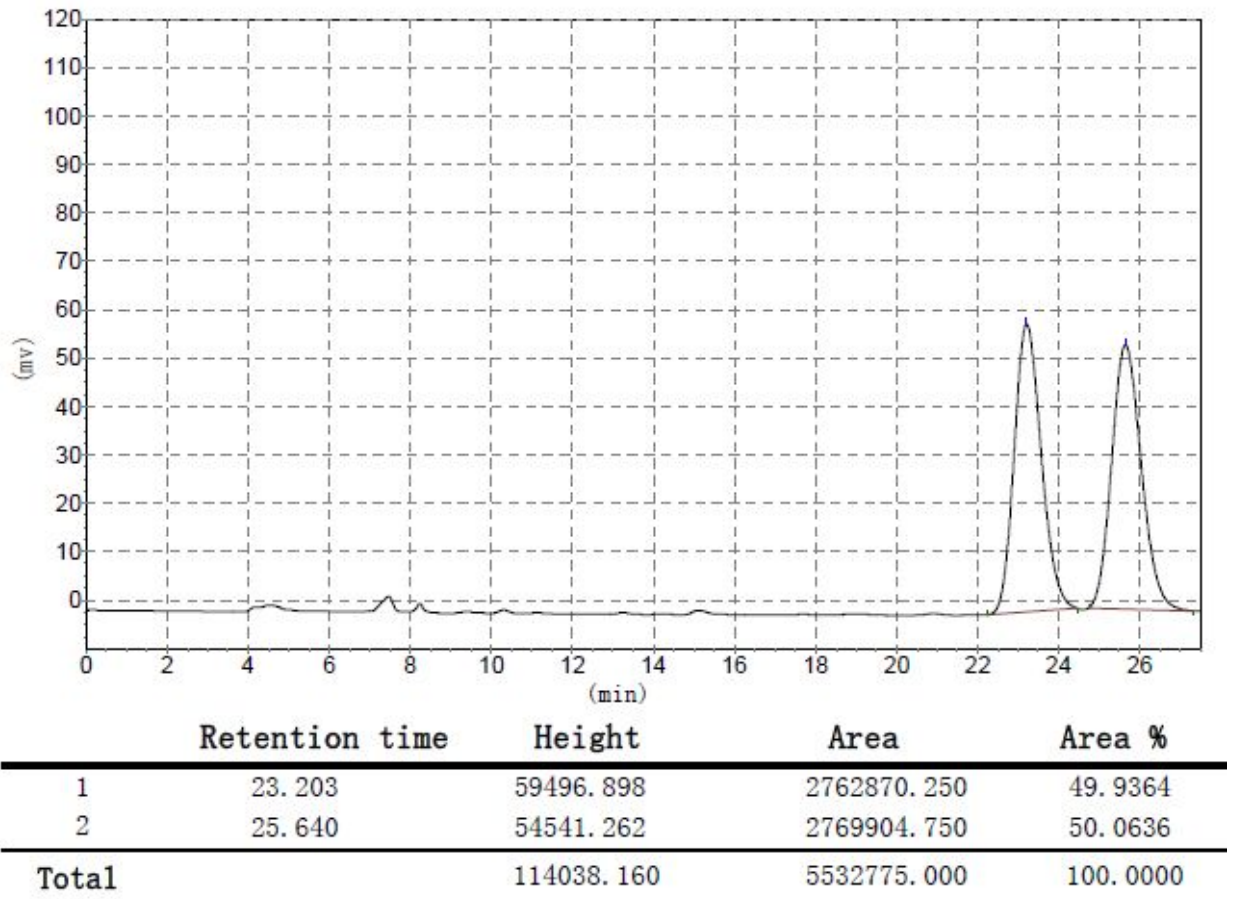

\section{Chiral}

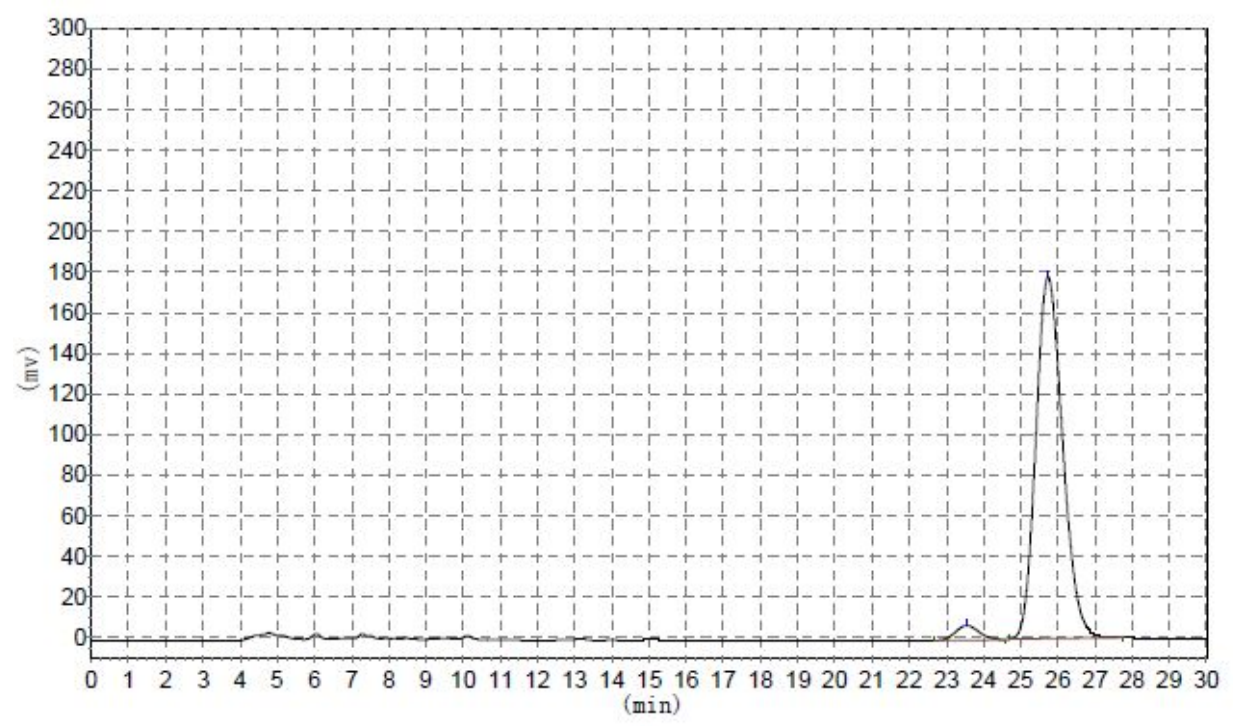

\begin{tabular}{ccccc} 
& Retention time & Height & Area & Area \% \\
\hline 1 & 23.547 & 7290.146 & 328969.813 & 3.5617 \\
2 & 25.738 & 178215.578 & 8907282.000 & 96.4383 \\
\hline Total & & 185505.724 & 9236251.813 & 100.0000
\end{tabular}


8j (Table 3, entry 10)<smiles>COc1oc([C@@H]2c3ccccc3CCN2N[AsH2])nc1-c1cccc(Br)c1</smiles>

\section{Racemic}

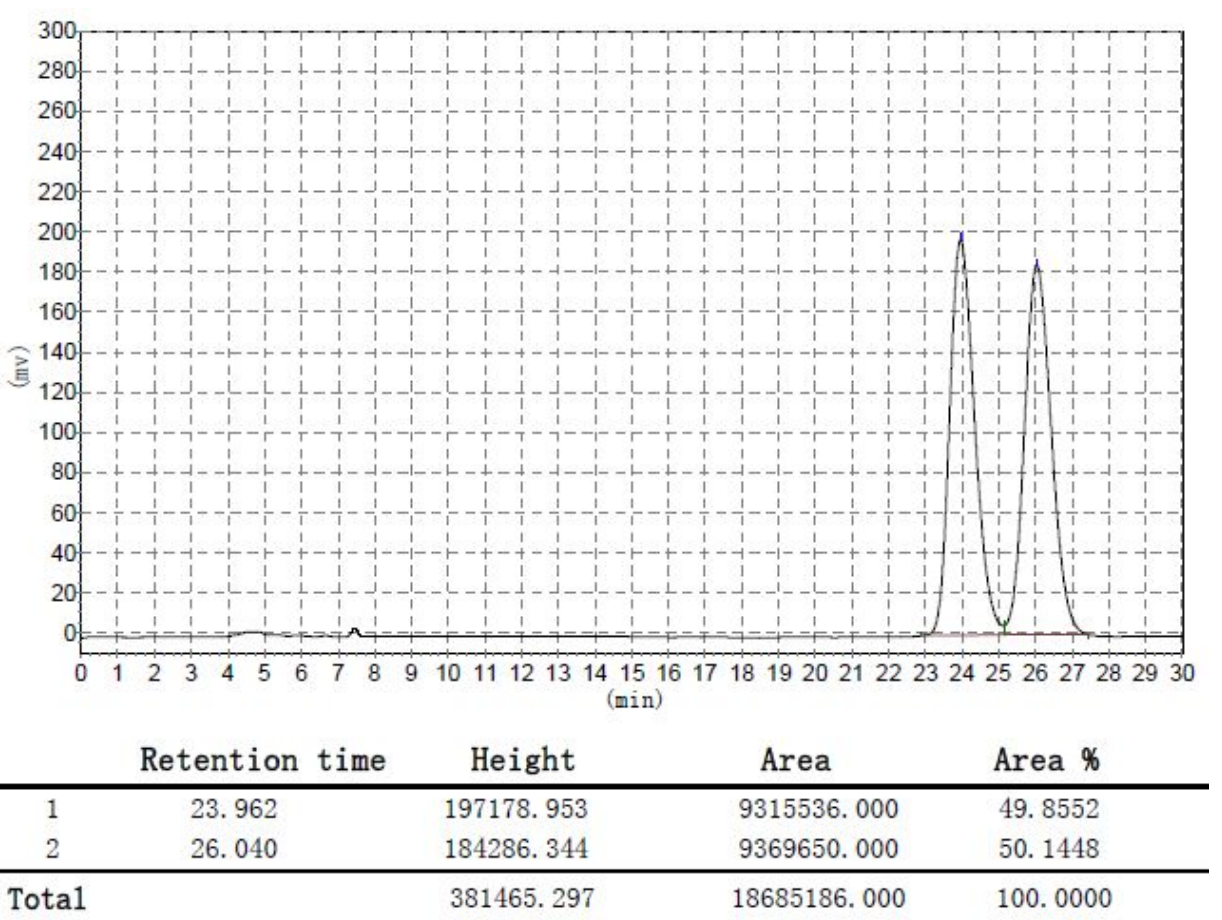

Chiral

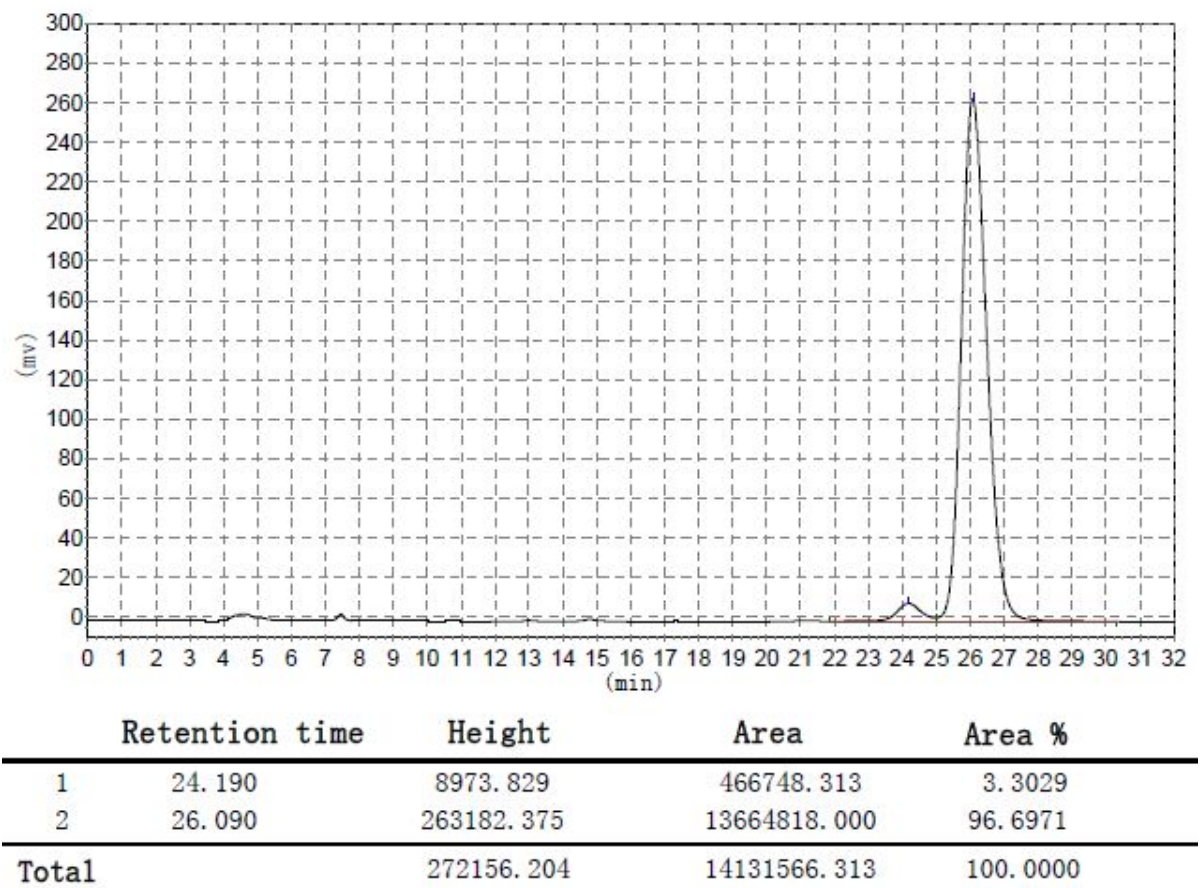




\section{8k (Table 3, entry 11)}<smiles>COc1oc([C@@H]2c3ccccc3CCN2N[As])nc1-c1cccc(C)c1</smiles>

\section{Racemic}

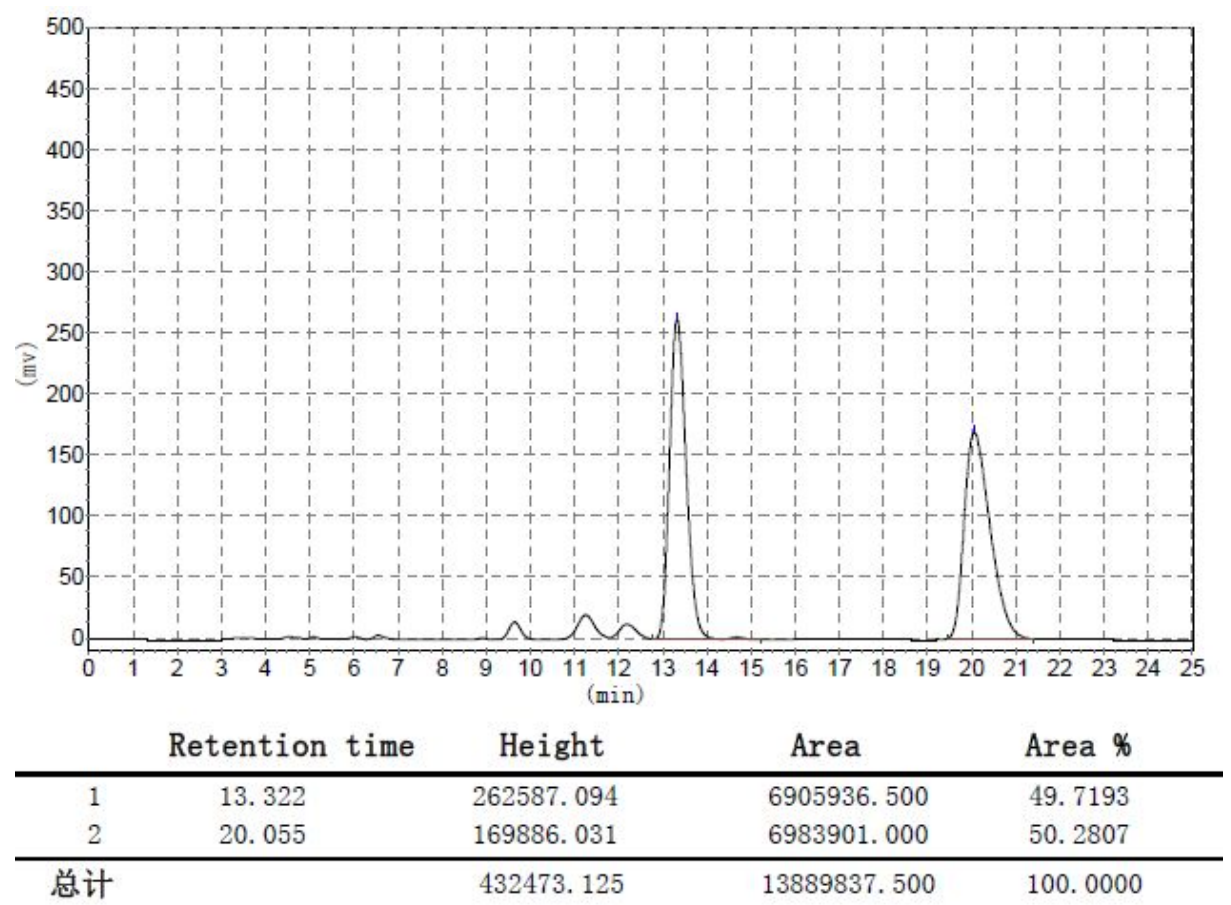

\section{Chiral}

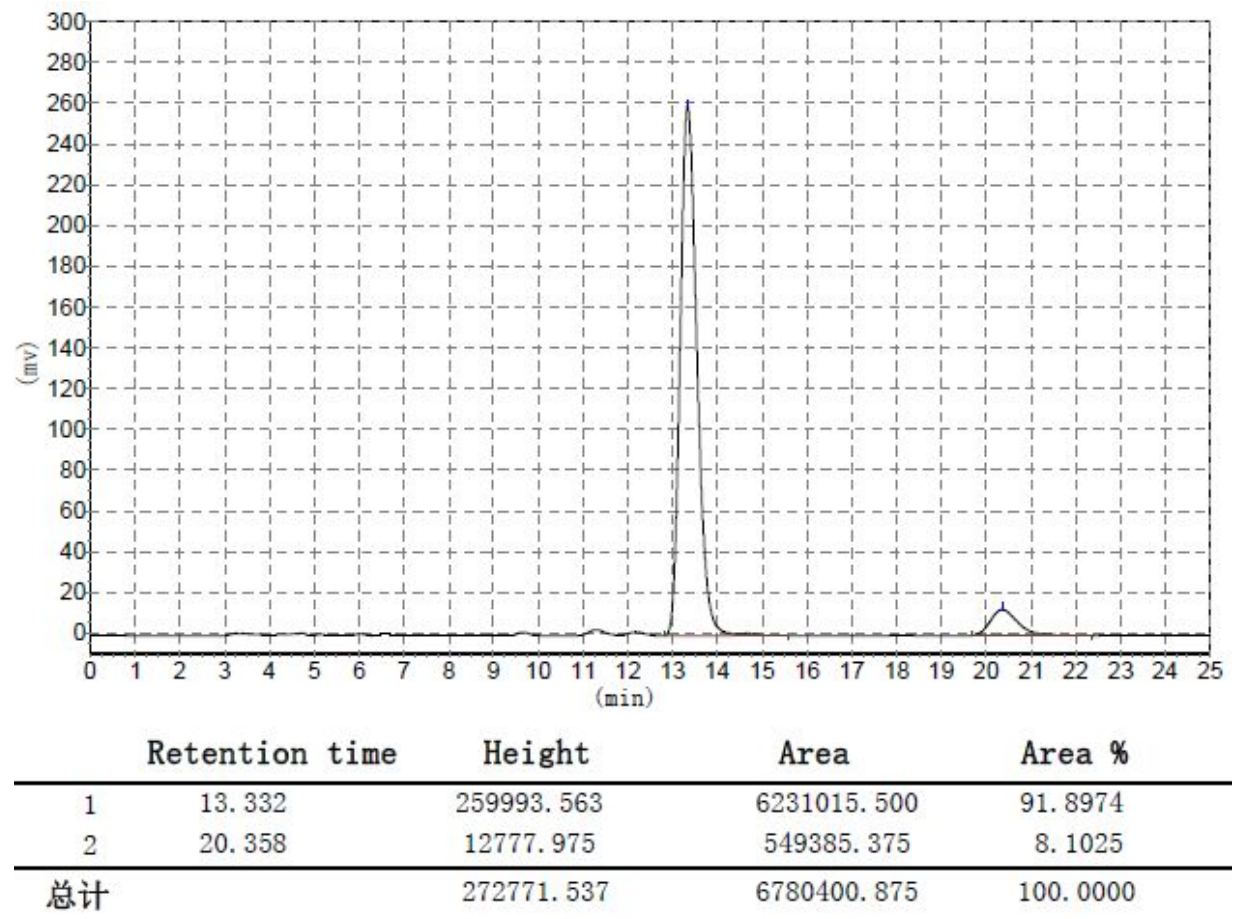


81 (Table 3, entry 12)<smiles>COc1oc(-c2ccccc2F)nc1C1c2ccccc2CCN1NC(F)(F)F</smiles>

Racemic

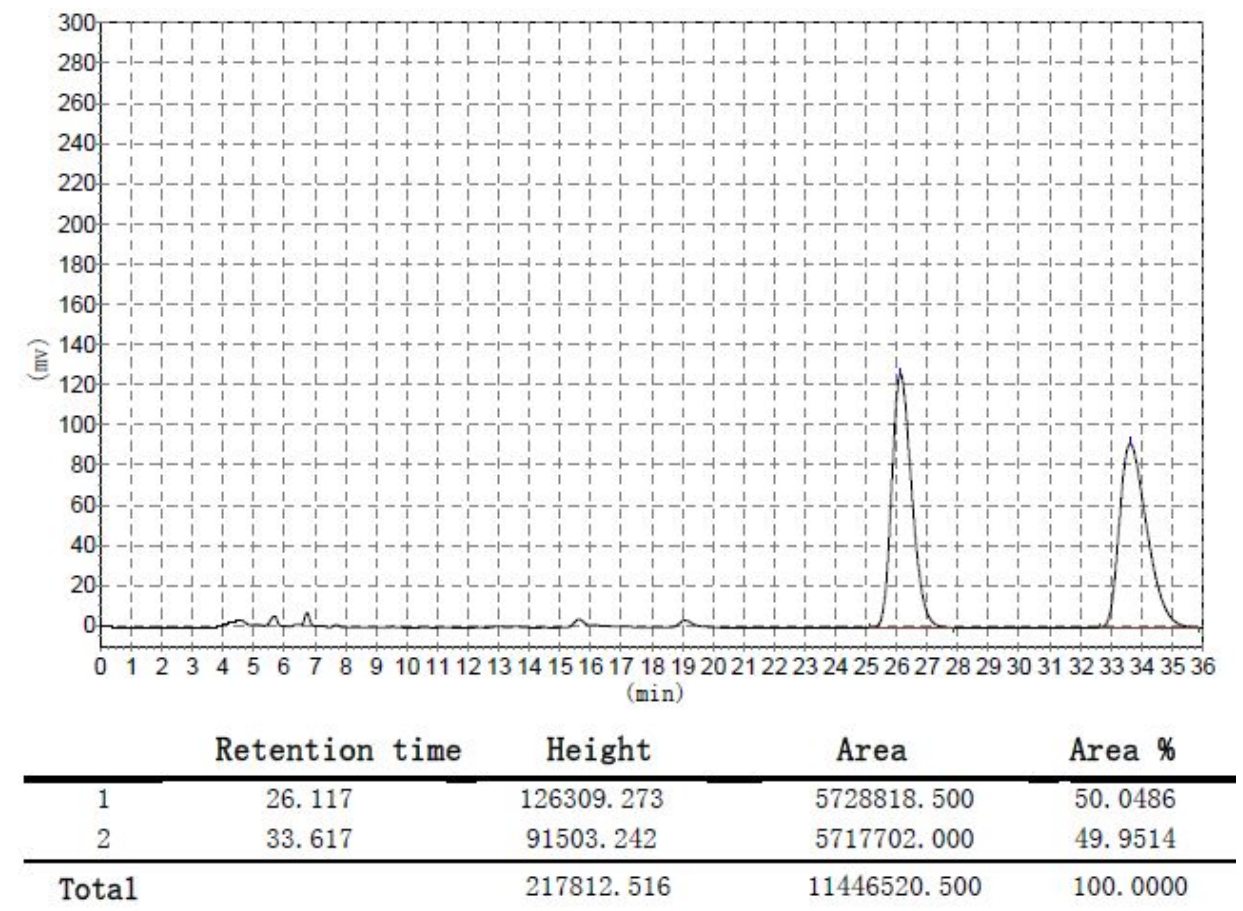

Chiral

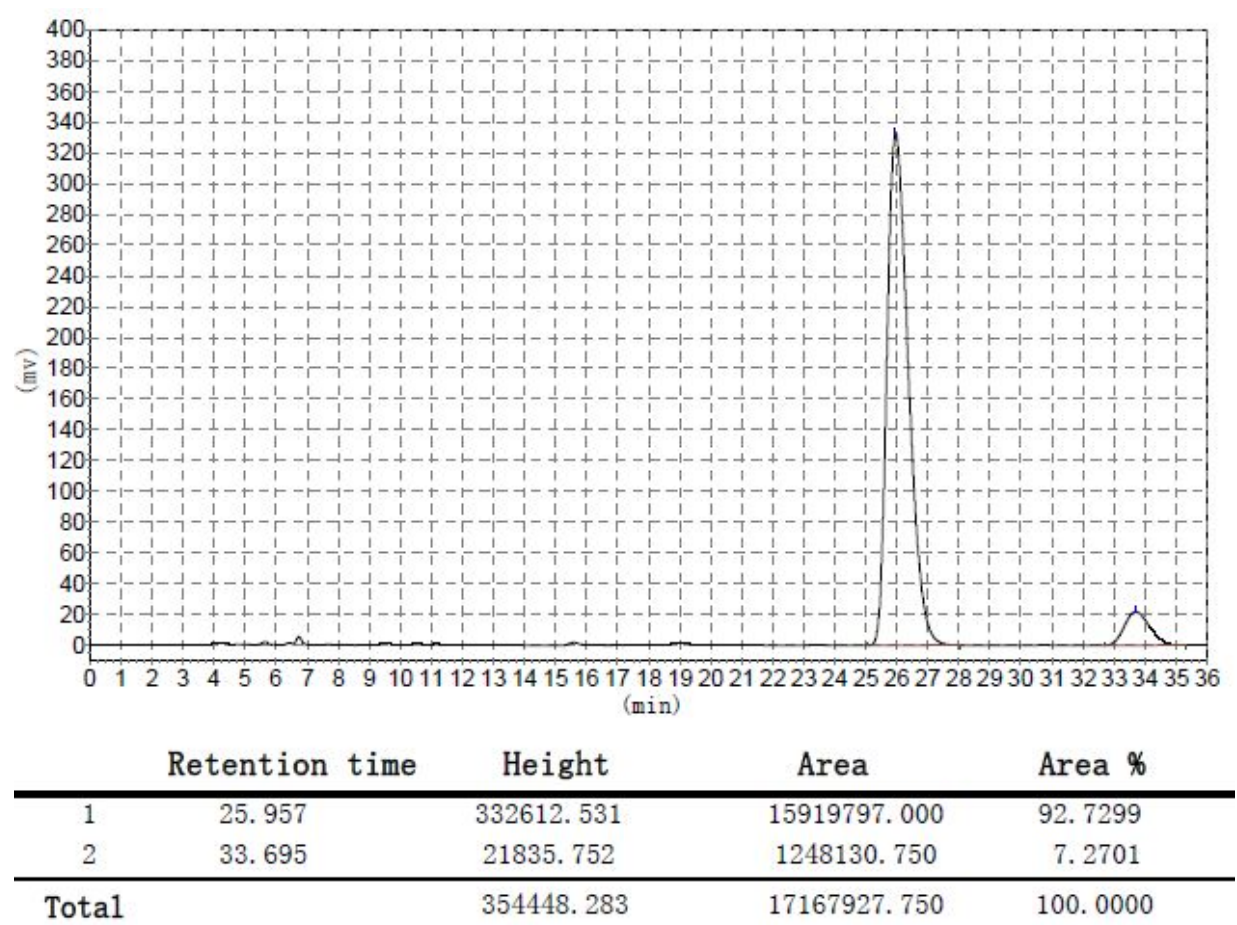




\section{8n (Table 3, entry 14)}<smiles>[AsH3-]NN1CCc2ccccc2[C@@H]1c1nc(-c2ccccc2)c(OCc2ccccc2)o1</smiles>

\section{Racemic}

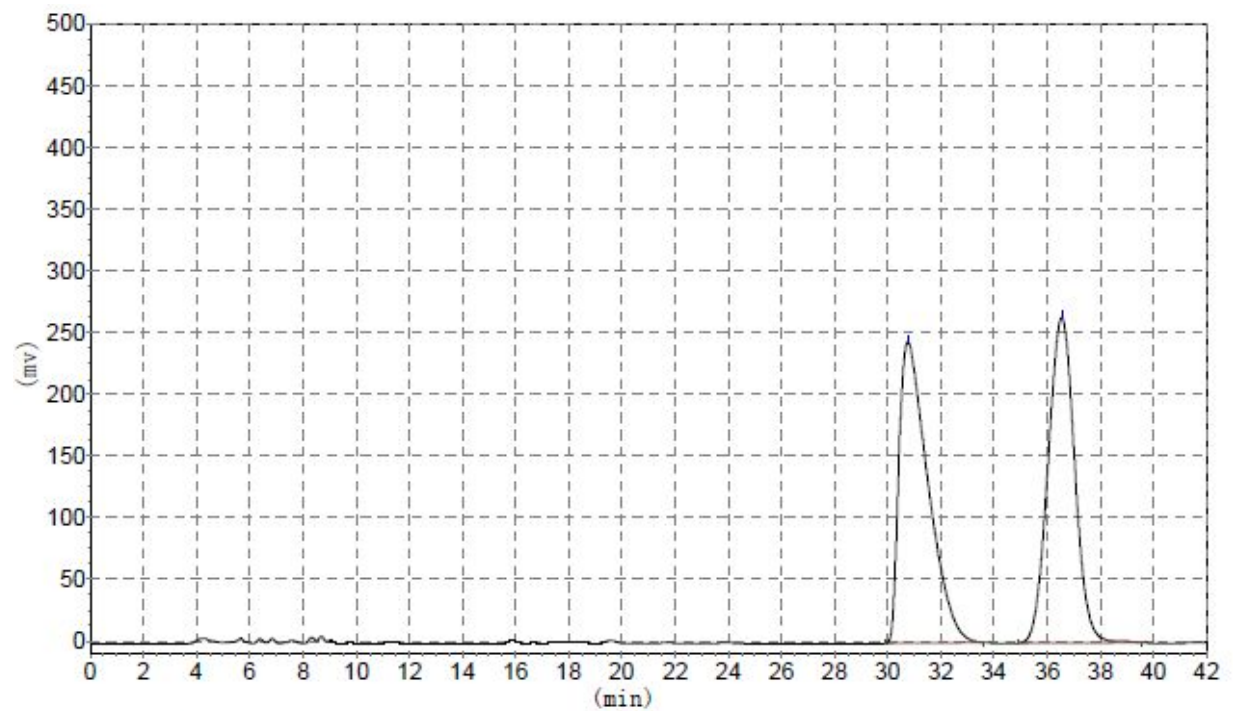

\begin{tabular}{ccccc} 
& Retention time & Height & Area & Area \% \\
\hline 1 & 30.757 & 243956.578 & 18505852.000 & 50.1522 \\
2 & 36.548 & 263631.656 & 18393498.000 & 49.8478 \\
\hline Tota1 & & 507588.234 & 36899350.000 & 100.0000
\end{tabular}

\section{Chiral}

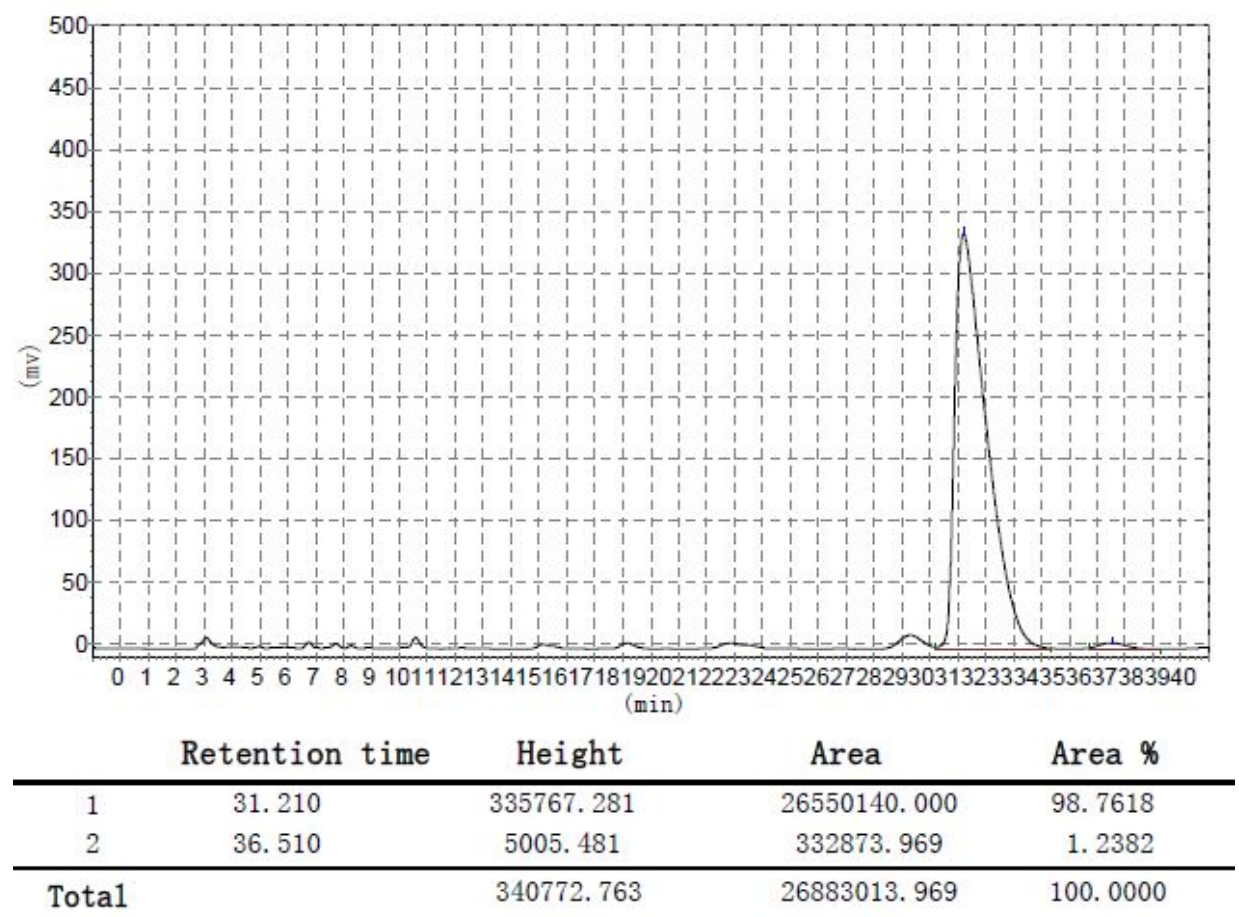




\section{0 (Table 3, entry 15)}<smiles>CC(C)(C)Oc1oc([C@@H]2c3ccccc3CCN2N[AsH])nc1-c1ccccc1</smiles>

\section{Racemic}

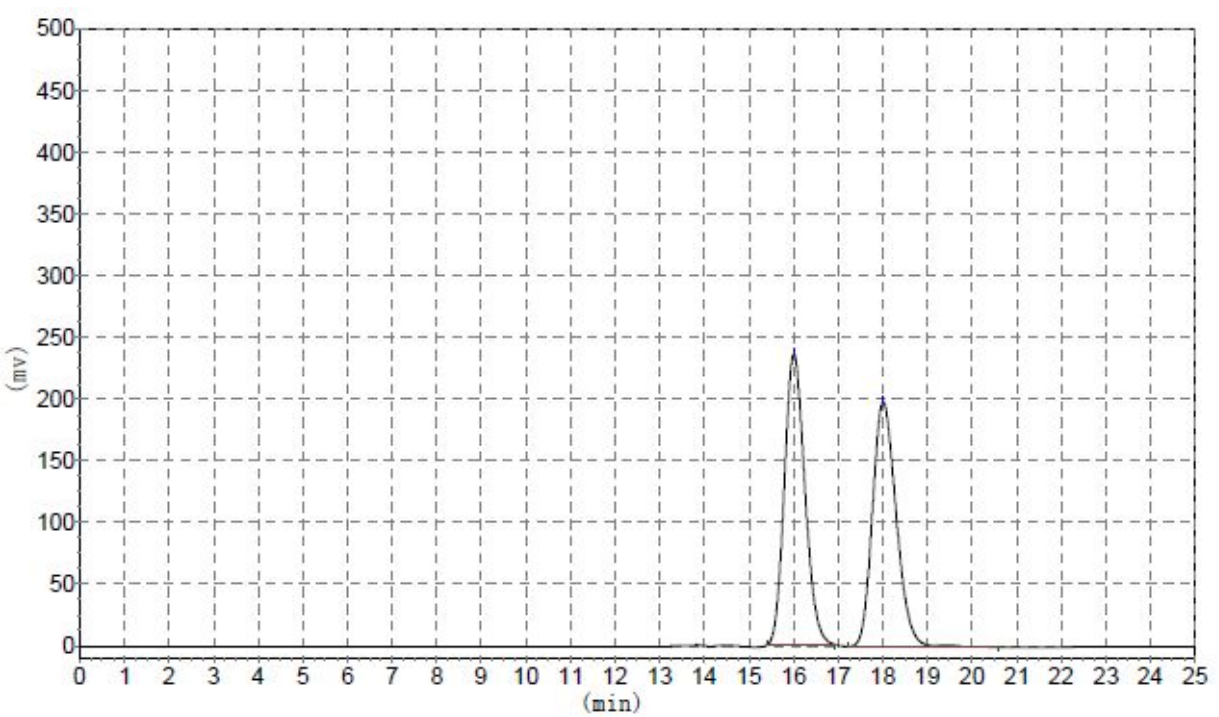

\begin{tabular}{ccccc} 
& Retention time & Height & Area & Area \% \\
\hline 1 & 16.003 & 235677.359 & 7530577.500 & 50.4235 \\
2 & 18.007 & 198812.719 & 7404079.000 & 49.5765 \\
\hline Total & & 434490.078 & 14934656.500 & 100.0000
\end{tabular}

\section{Chiral}

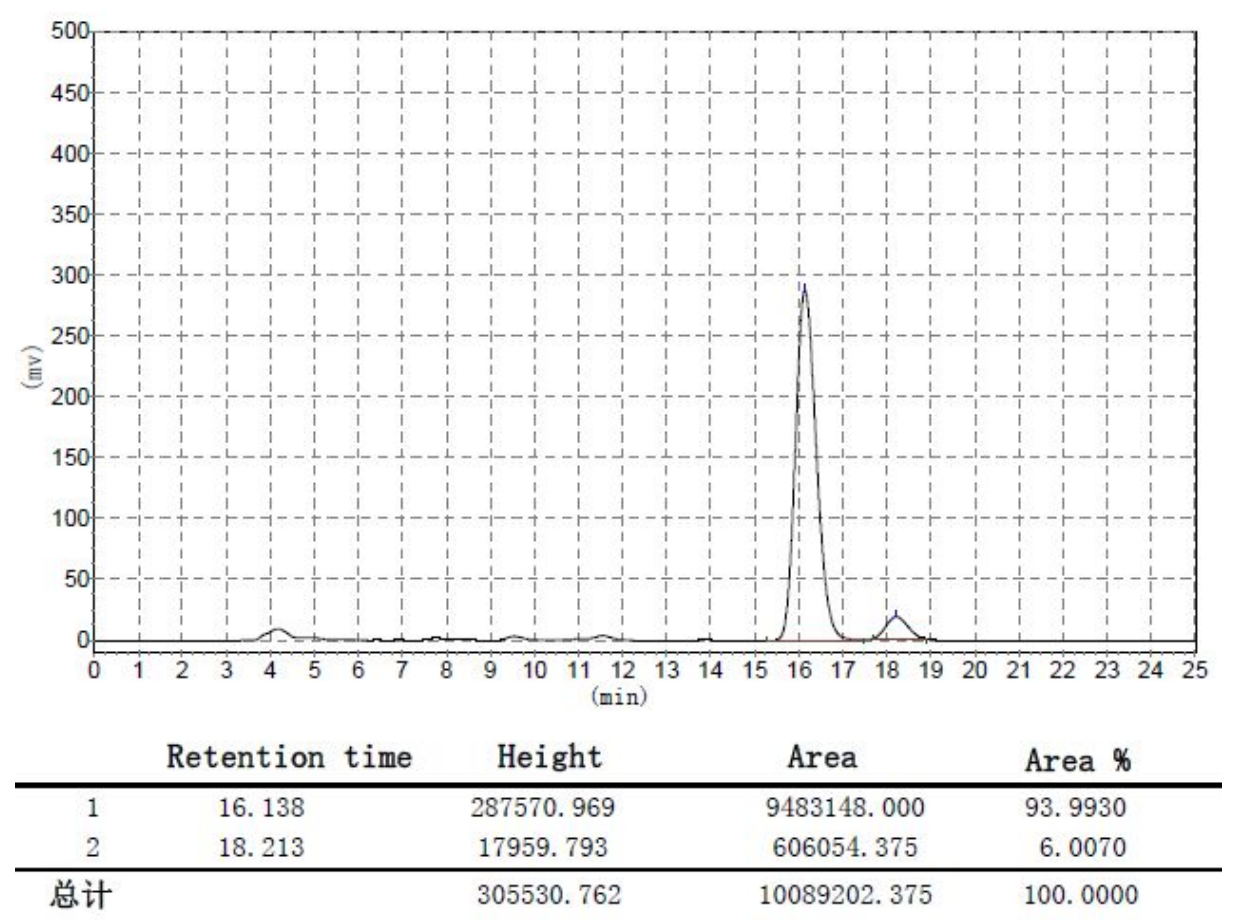




\section{8q (Table 3, entry 17)}<smiles>FNNN1CCc2ccccc2[C@H]1c1nc(-c2ccccc2)c(N2CCOCC2)o1</smiles>

\section{Racemic}

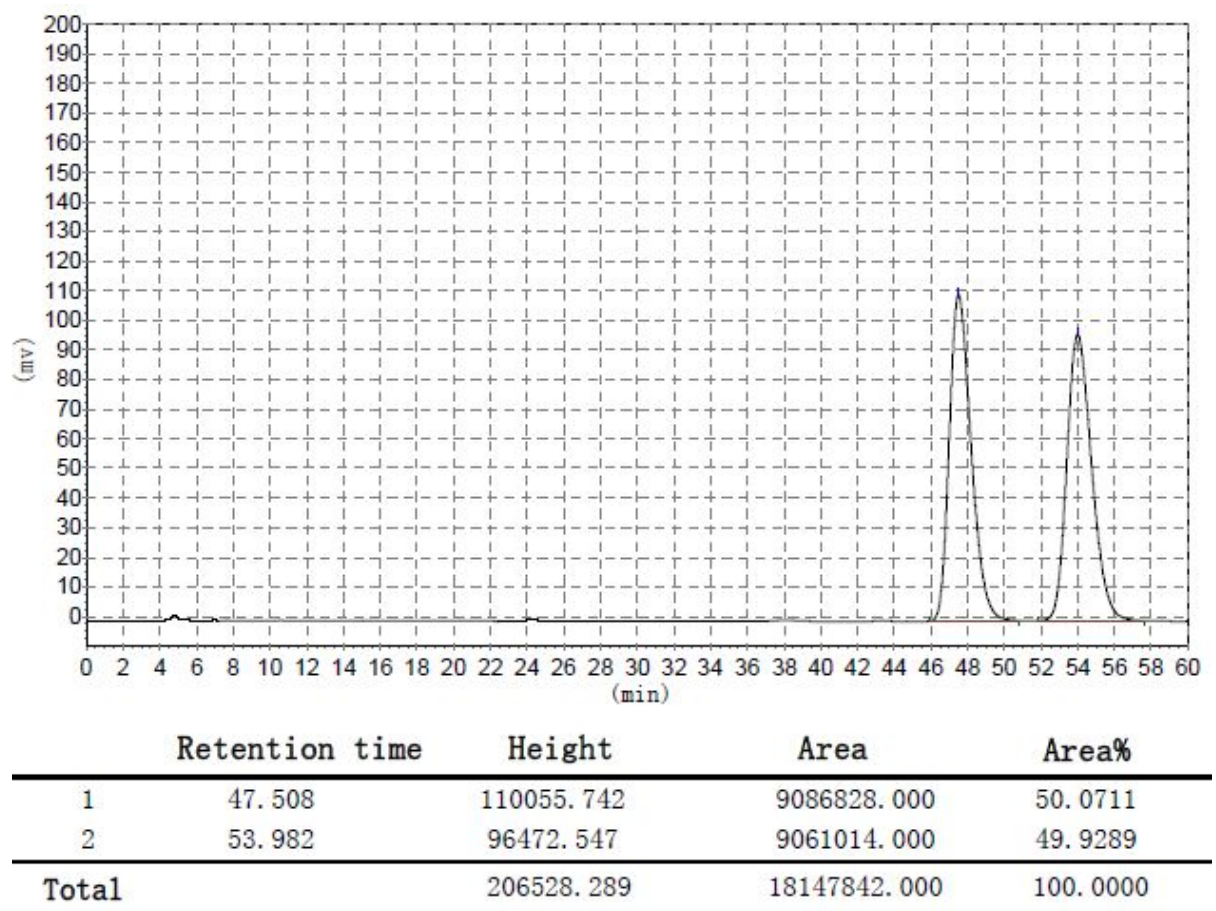

\section{Chiral}

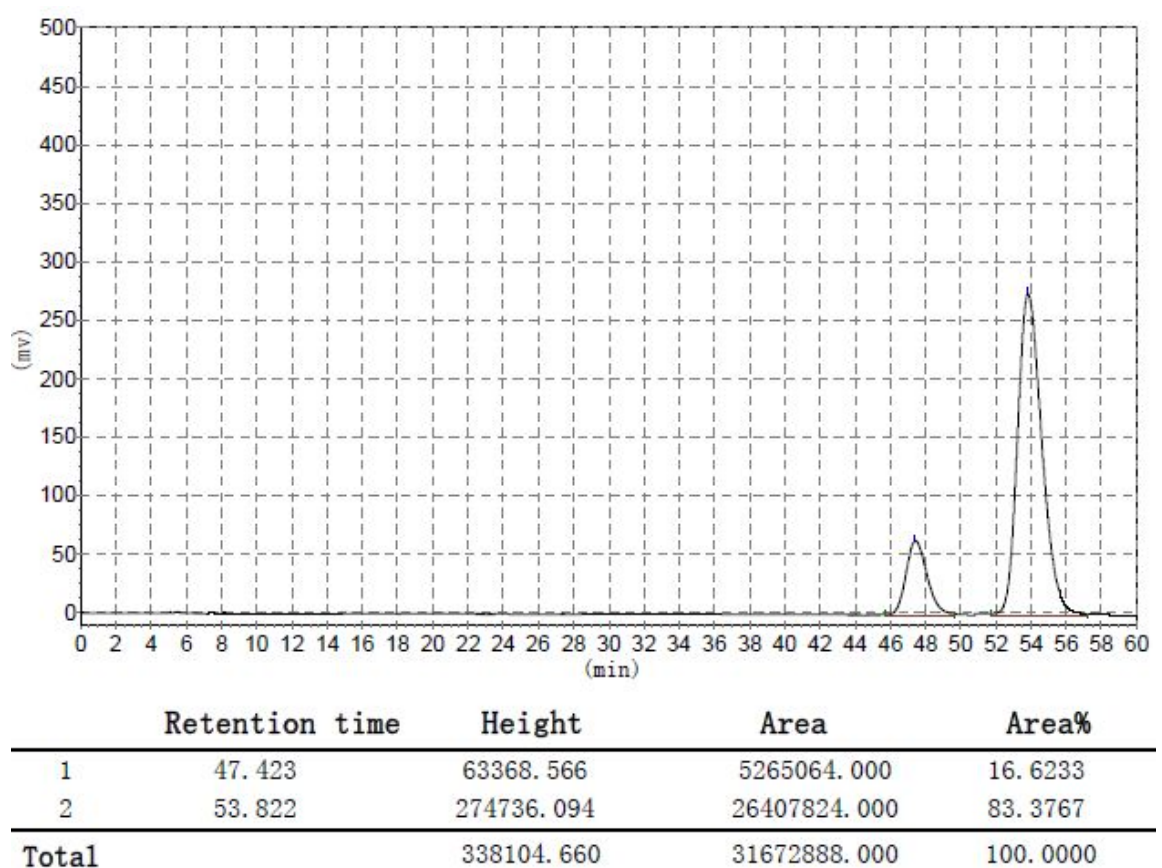




\section{8r (Table 4, entry 1)}<smiles>COc1oc([C@H]2c3ccccc3CCN2NS(=O)(=O)c2cccc([N+](=O)[O-])c2)nc1-c1ccccc1</smiles>

\section{Racemic}

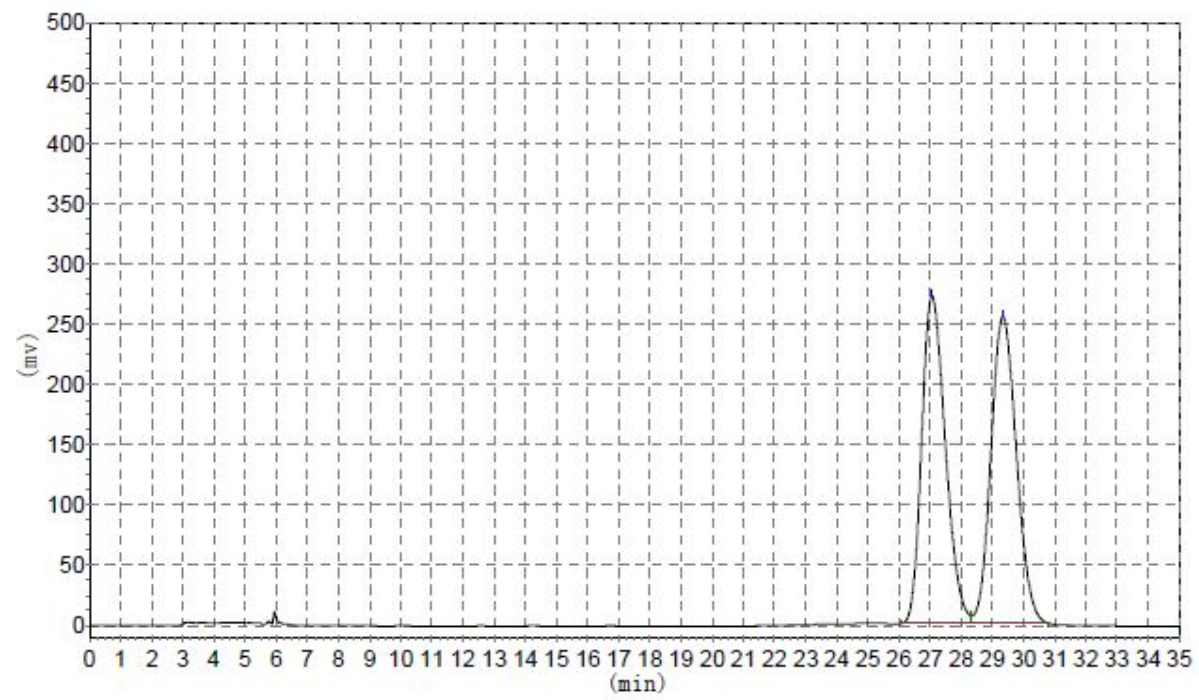

\begin{tabular}{ccccc} 
& Retention time & Height & Area & Area \% \\
\hline 1 & 27.060 & 271859.250 & 14355318.000 & 49.8151 \\
2 & 29.335 & 254835.500 & 14461885.000 & 50.1849 \\
\hline Total & & 526694.750 & 28817203.000 & 100.0000
\end{tabular}

\section{Chiral}

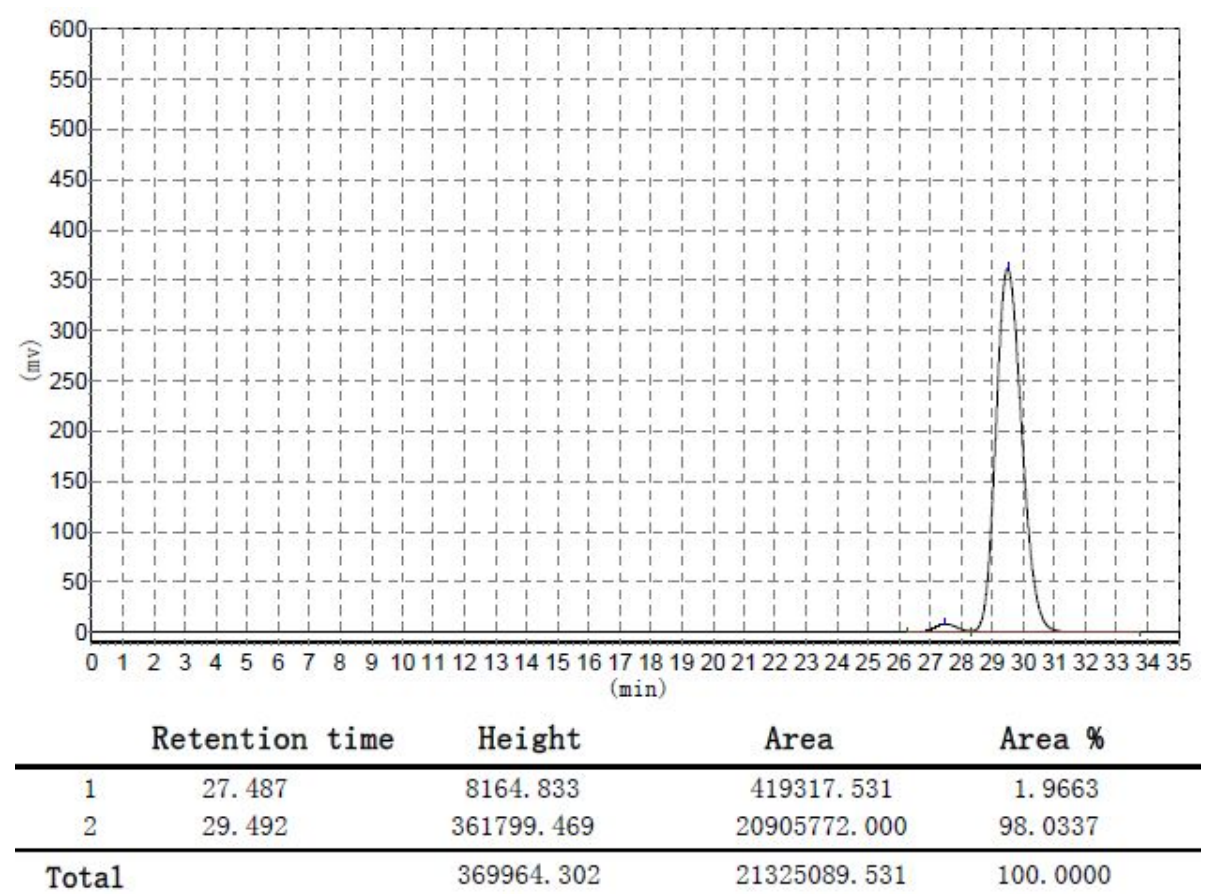




\section{8s (Table 4, entry 2)}<smiles></smiles>

\section{Racemic}

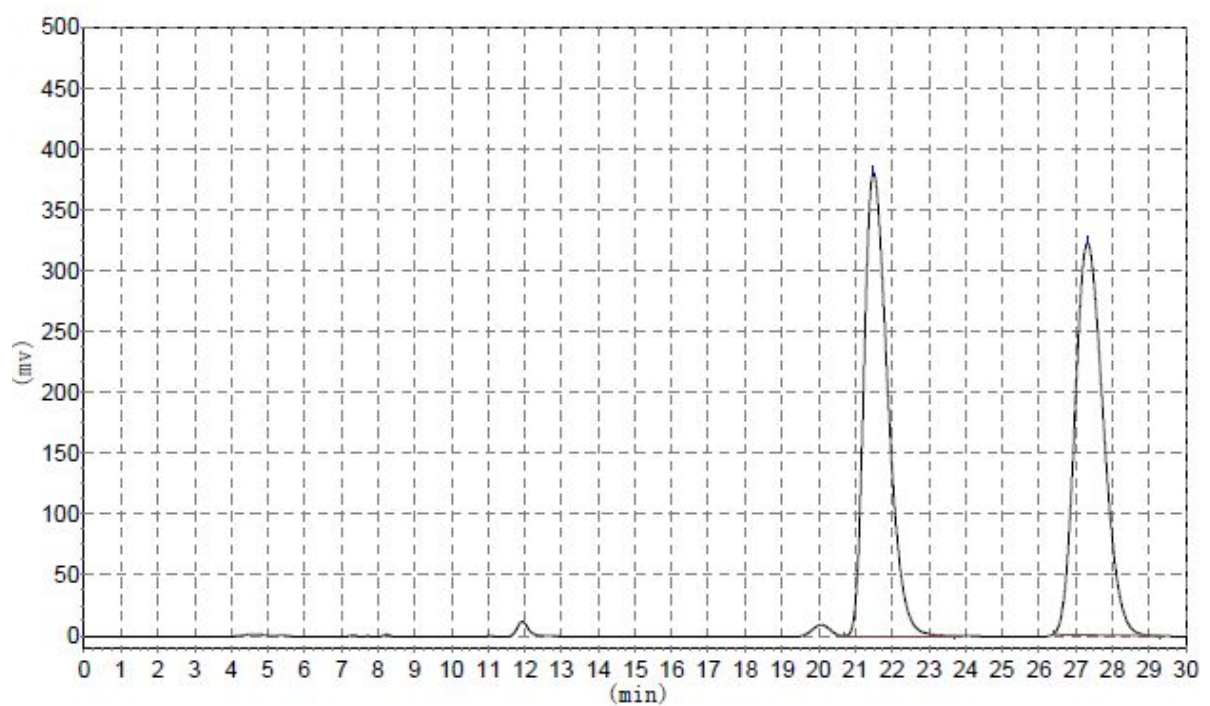

\begin{tabular}{ccccc} 
& Retention time & Height & Area & Area \% \\
\hline 1 & 21.495 & 381180.344 & 17123026.000 & 49.8336 \\
2 & 27.320 & 322253.938 & 17237348.000 & 50.1664 \\
\hline Tota1 & & 703434.281 & 34360374.000 & 100.0000
\end{tabular}

\section{Chiral}

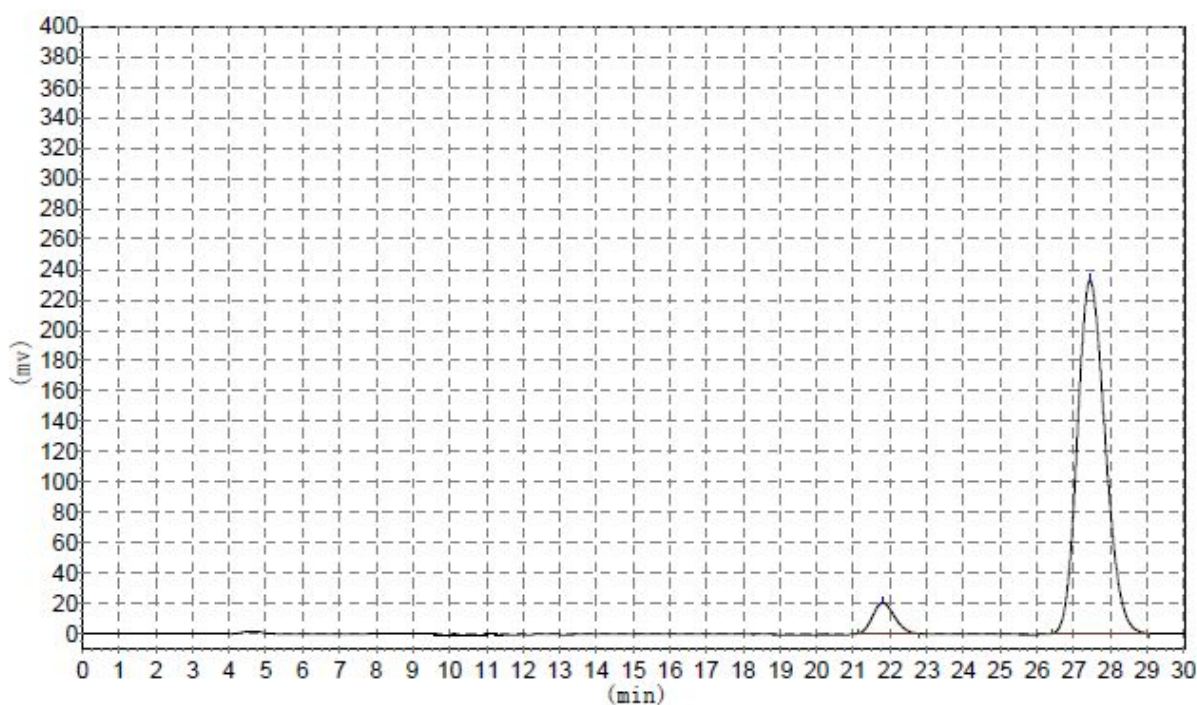

\begin{tabular}{ccccc} 
& Retention time & Height & Area & Area \% \\
\hline 1 & 21.798 & 20389.008 & 841543.375 & 6.4311 \\
2 & 27.448 & 232734.984 & 12244000.000 & 93.5689 \\
\hline Total & & 253123.992 & 13085543.375 & 100.0000
\end{tabular}




\section{8t (Table 4, entry 3)}<smiles>COc1ccc(S(=O)(=O)NN2CCc3ccccc3C2c2nc(-c3ccccc3)c(OC)o2)cc1</smiles>

\section{Racemic}

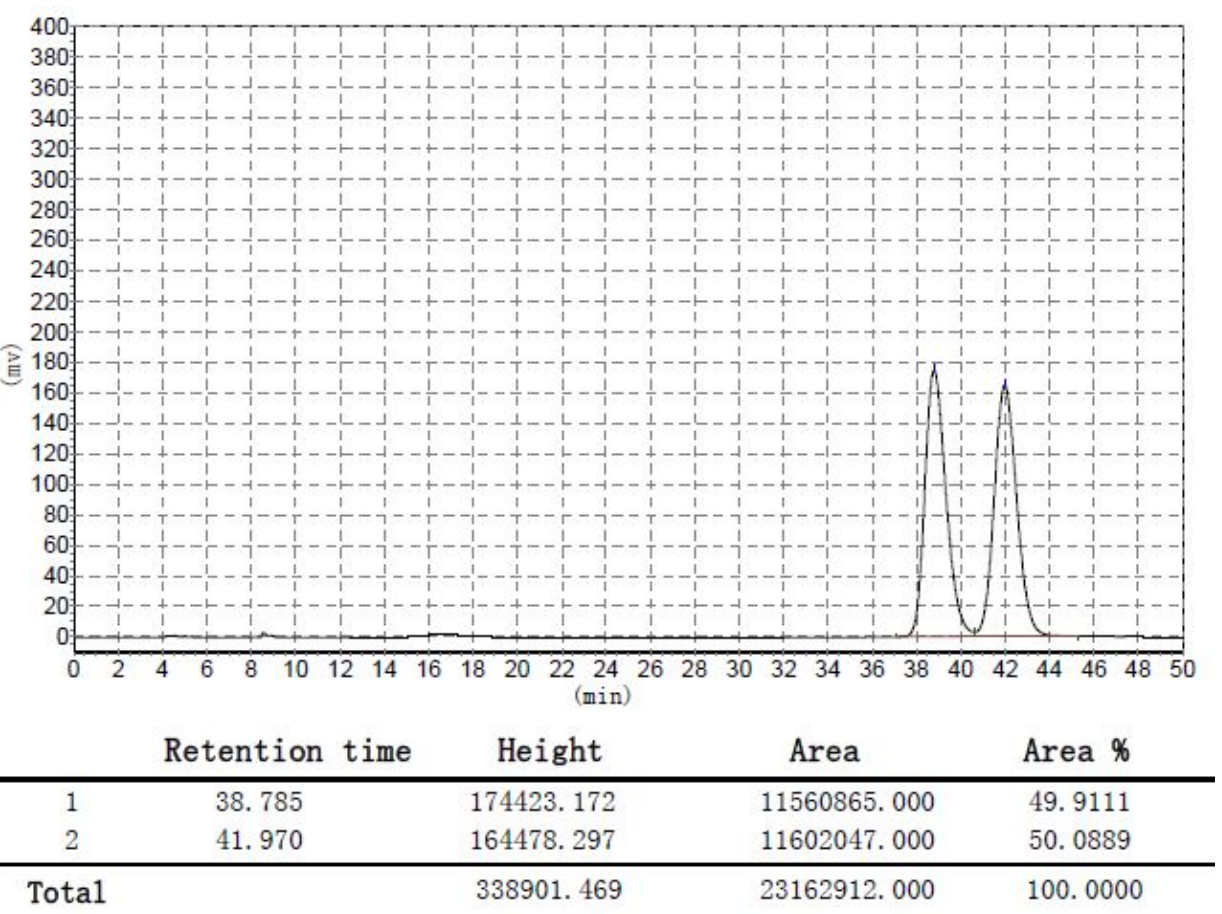

\section{Chiral}

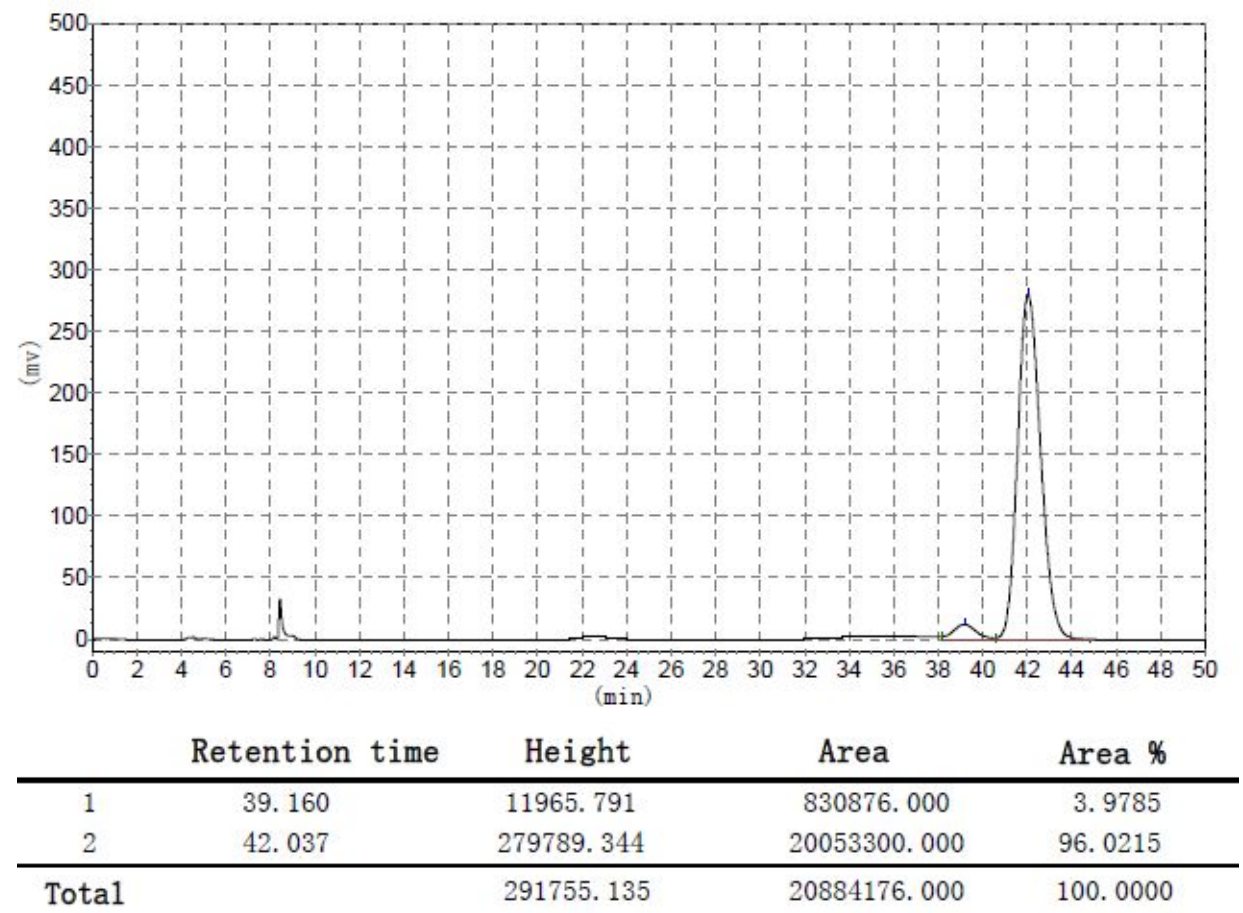




\section{8u (Table 4, entry 4)}<smiles>COc1oc([C@@H]2c3ccccc3CCN2NS(=O)(=O)Cc2ccccc2)nc1-c1ccccc1</smiles>

\section{Racemic}

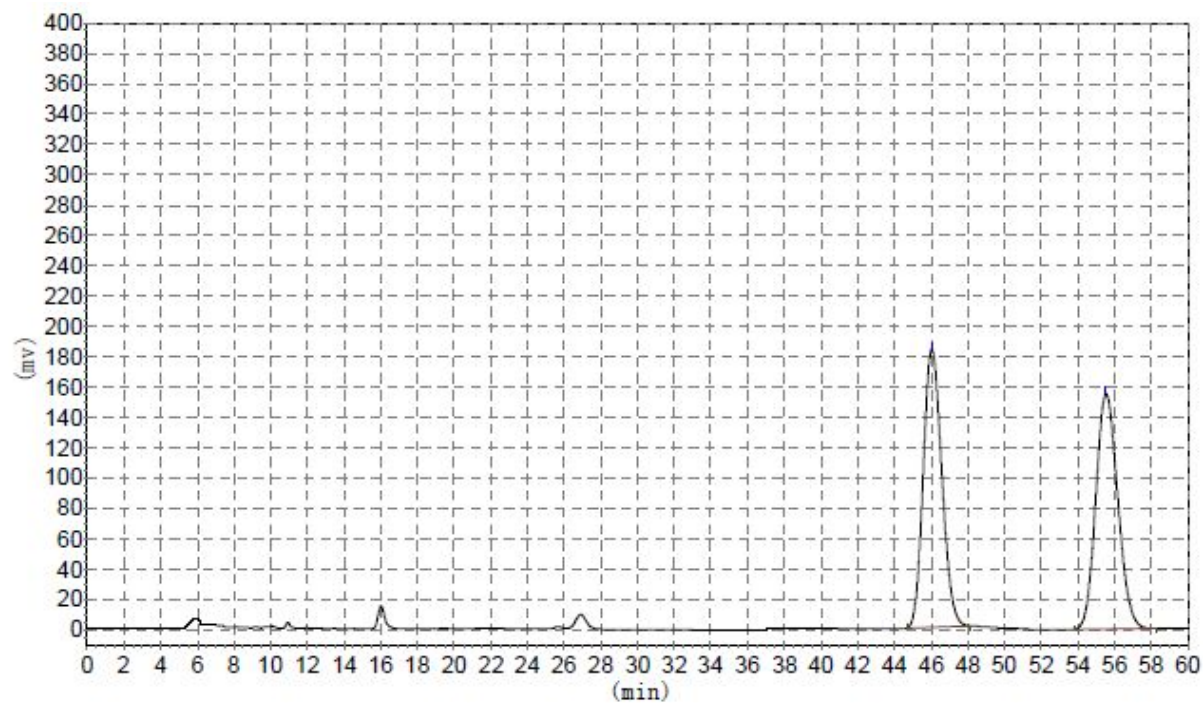

\begin{tabular}{ccccc} 
& Retention time & Height & Area & Area \% \\
\hline 1 & 46.040 & 183671.422 & 12912639.000 & 49.9705 \\
2 & 55.540 & 155255.719 & 12927900.000 & 50.0295 \\
\hline Tota1 & & 338927.141 & 25840539.000 & 100.0000
\end{tabular}

\section{Chiral}

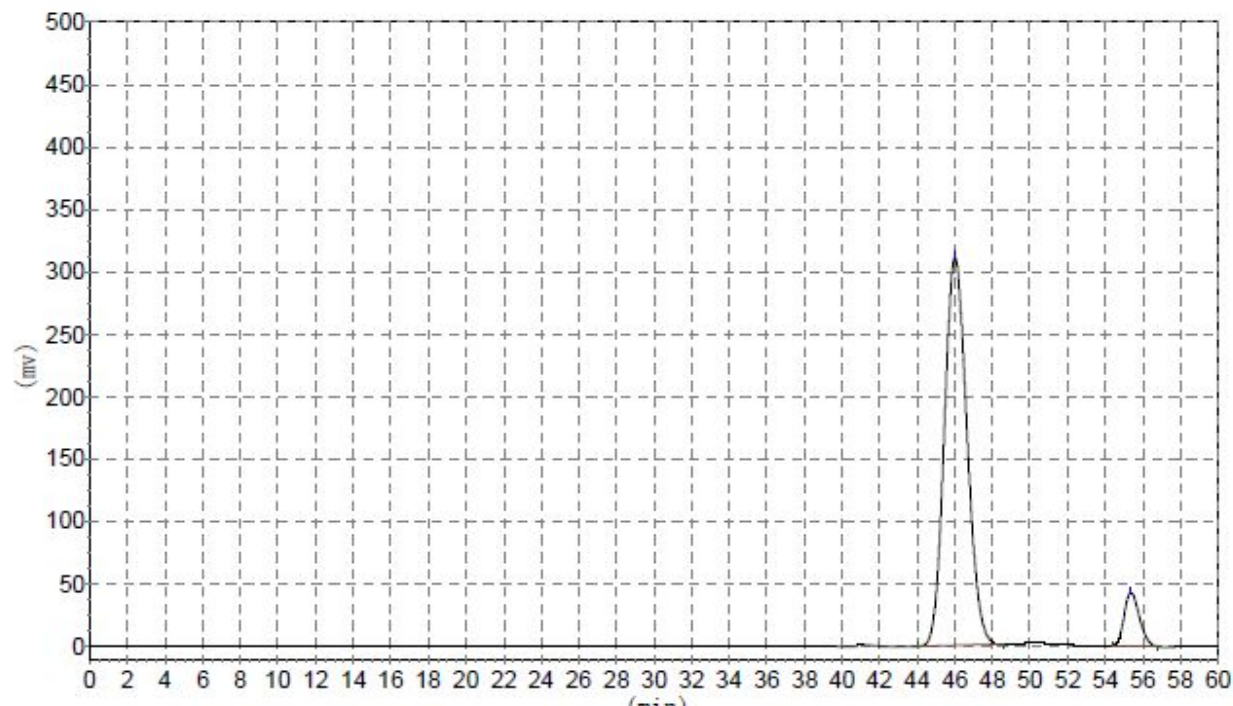

$$
\text { (min) }
$$

\begin{tabular}{ccccc} 
& Retention time & Height & Area & Area \% \\
\hline 1 & 46.002 & 310674.500 & 25892176.000 & 91.3654 \\
2 & 55.360 & 43248.539 & 2446977.000 & 8.6346 \\
\hline Total & & 353923.039 & 28339153.000 & 100.0000
\end{tabular}




\section{8v (Table 4, entry 5)}<smiles></smiles>

\section{Racemic}

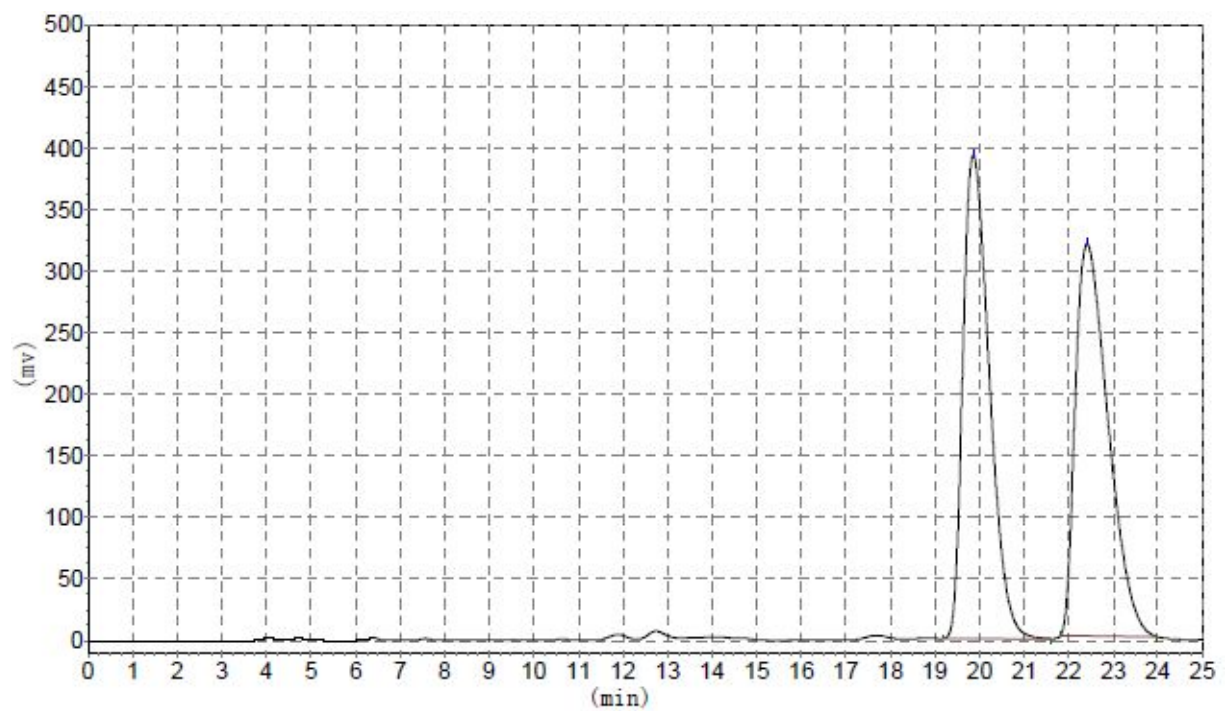

\begin{tabular}{ccccc} 
& Retention time & Height & Area & Area \% \\
\hline 1 & 19.858 & 392982.344 & 16177926.000 & 49.8298 \\
2 & 22.410 & 318239.750 & 16288436.000 & 50.1702 \\
\hline Tota1 & & 711222.094 & 32466362.000 & 100.0000
\end{tabular}

\section{Chiral}

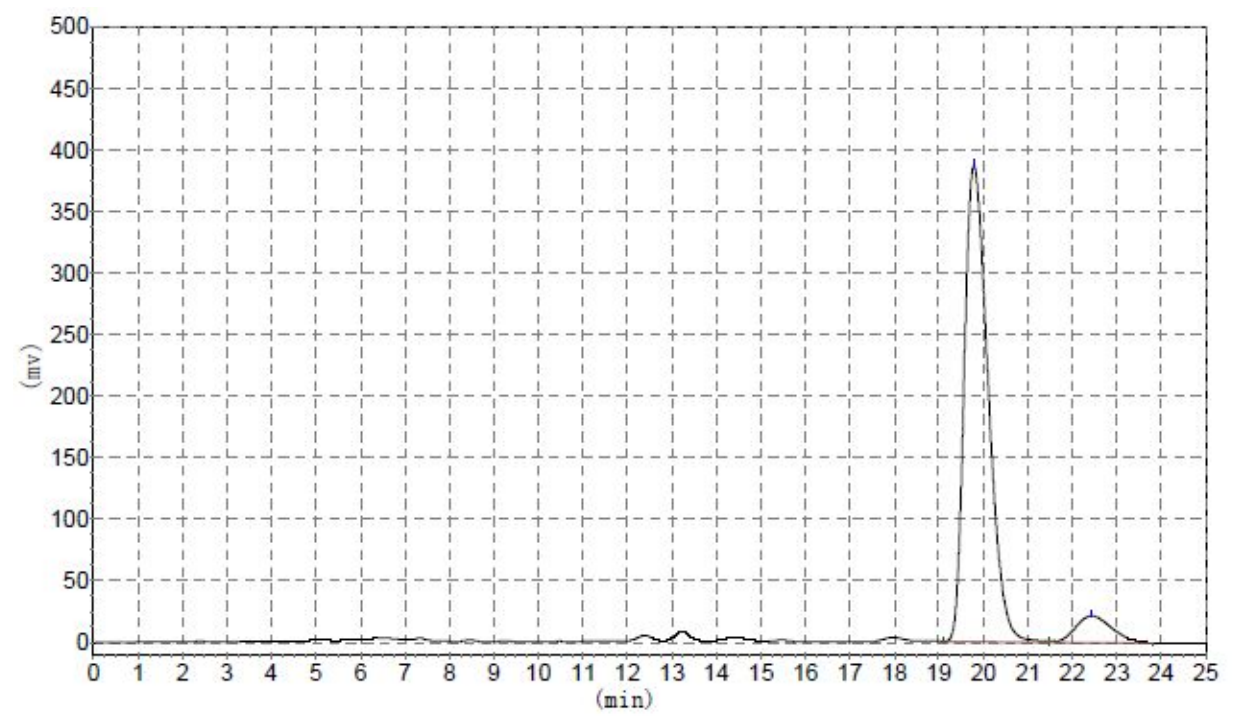

\begin{tabular}{ccccc} 
& Retention time & Height & Area & Area \% \\
\hline 1 & 19.787 & 386572.156 & 14472545.619 & 92.5873 \\
2 & 22.432 & 21653.162 & 1158697.131 & 7.4127 \\
\hline Total & & 408225.318 & 15631242.750 & 100.0000
\end{tabular}




\section{8w (Table 4, entry 6)}<smiles>COc1oc([C@@H]2c3cc(Cl)ccc3CCN2N[In])nc1-c1ccccc1</smiles>

\section{Racemic}

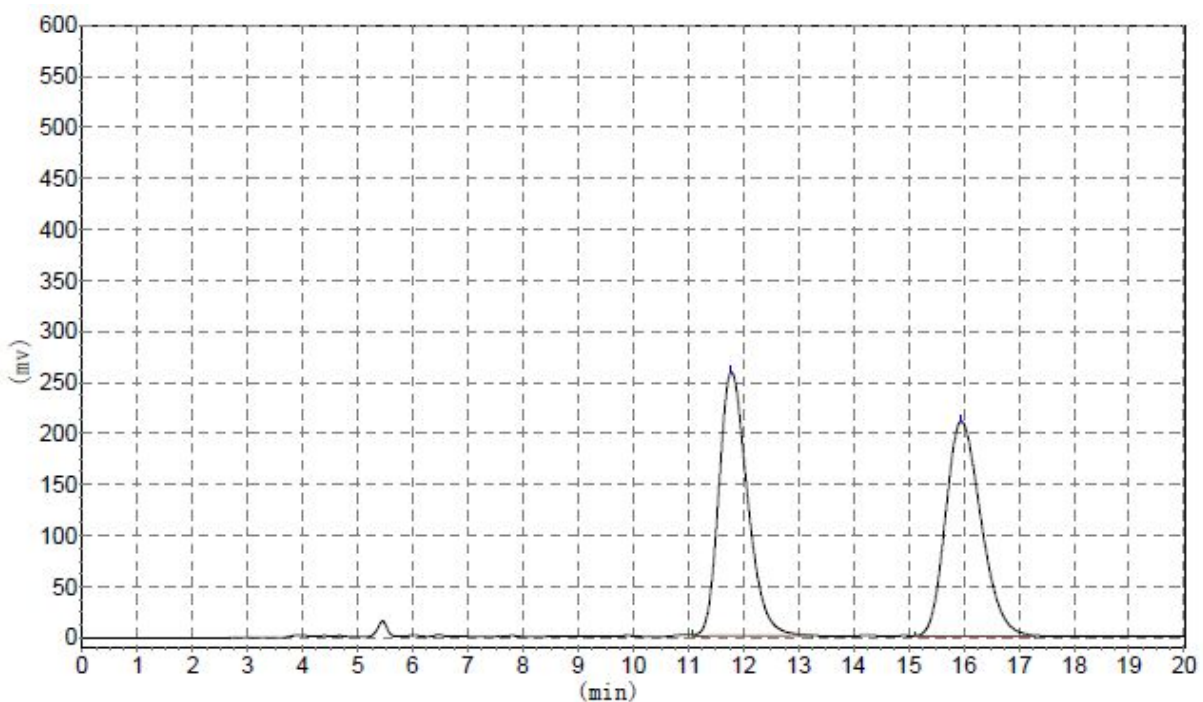

\begin{tabular}{ccccc} 
& Retention time & Height & Area & Area\% \\
\hline 1 & 11.780 & 258785.781 & 9332828.000 & 49.9930 \\
2 & 15.953 & 210175.438 & 9335426.000 & 50.0070 \\
\hline Total & & 468961.219 & 18668254.000 & 100.0000
\end{tabular}

\section{Chiral}

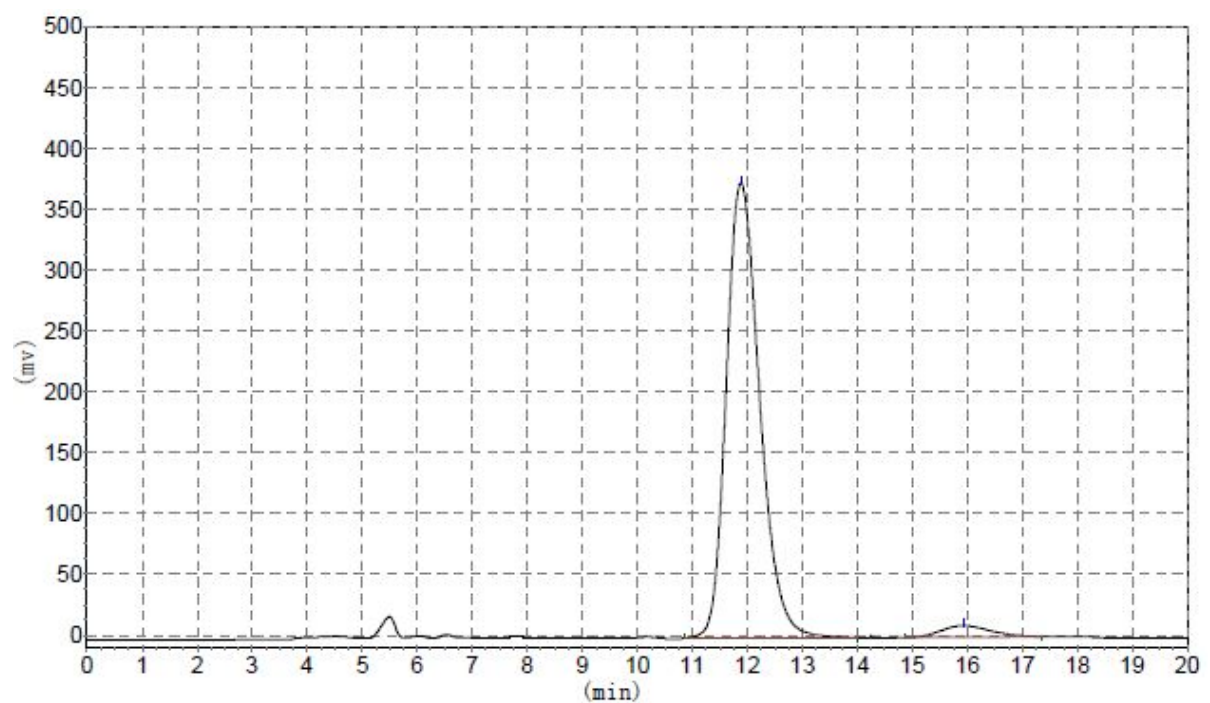

\begin{tabular}{ccccc} 
& Retention time & Height & Area & Area $\%$ \\
\hline 1 & 11.885 & 374083.844 & 15774572.000 & 96.3728 \\
2 & 15.920 & 9422.112 & 593711.625 & 3.6272 \\
\hline Total & & 383505.956 & 16368283.625 & 100.0000
\end{tabular}




\section{8x (Table 4, entry 7)}<smiles>[3H]NN1CCc2ccc(Br)cc2[C@H]1c1nc(-c2ccccc2)c(OC)o1</smiles>

\section{Racemic}

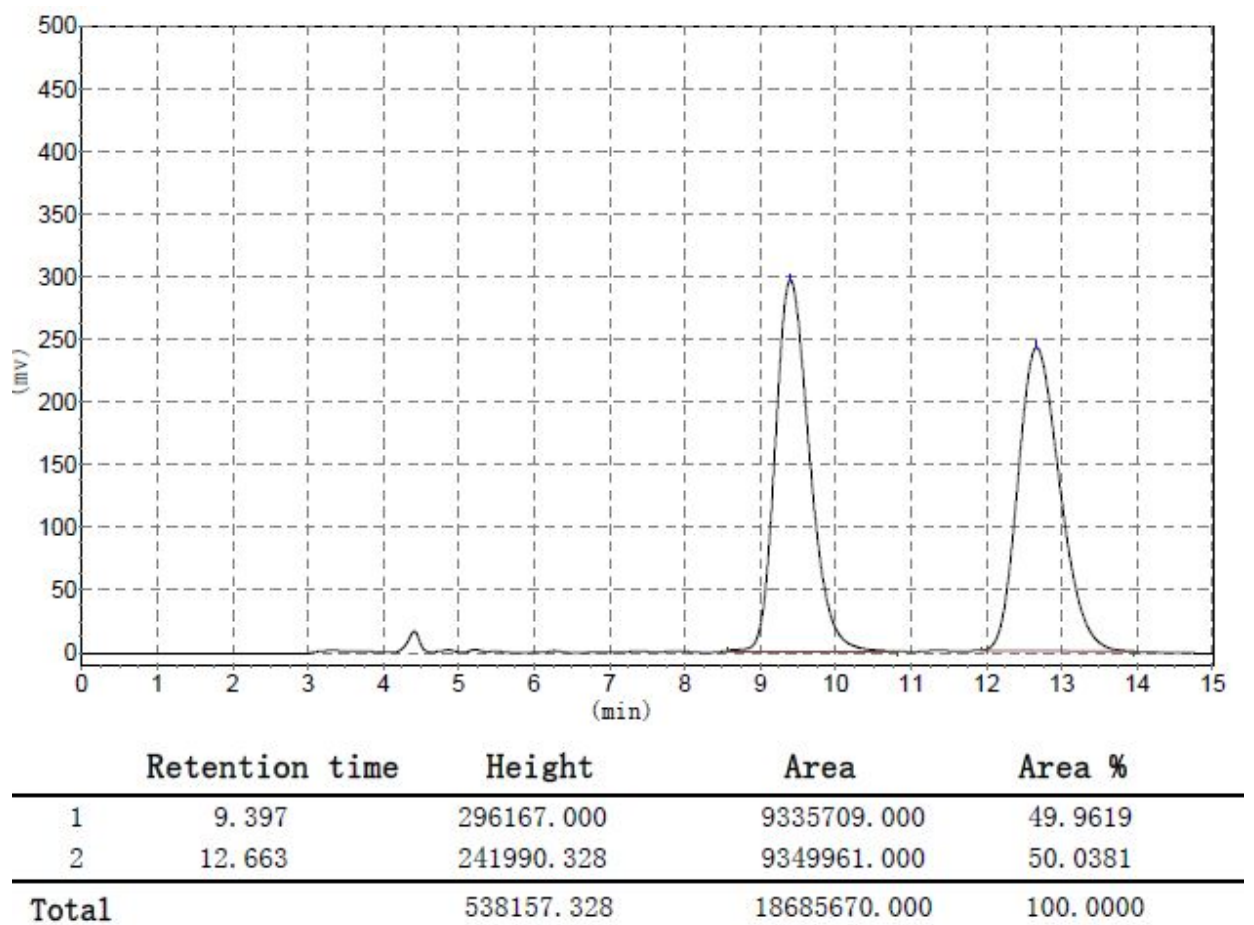

\section{Chiral}

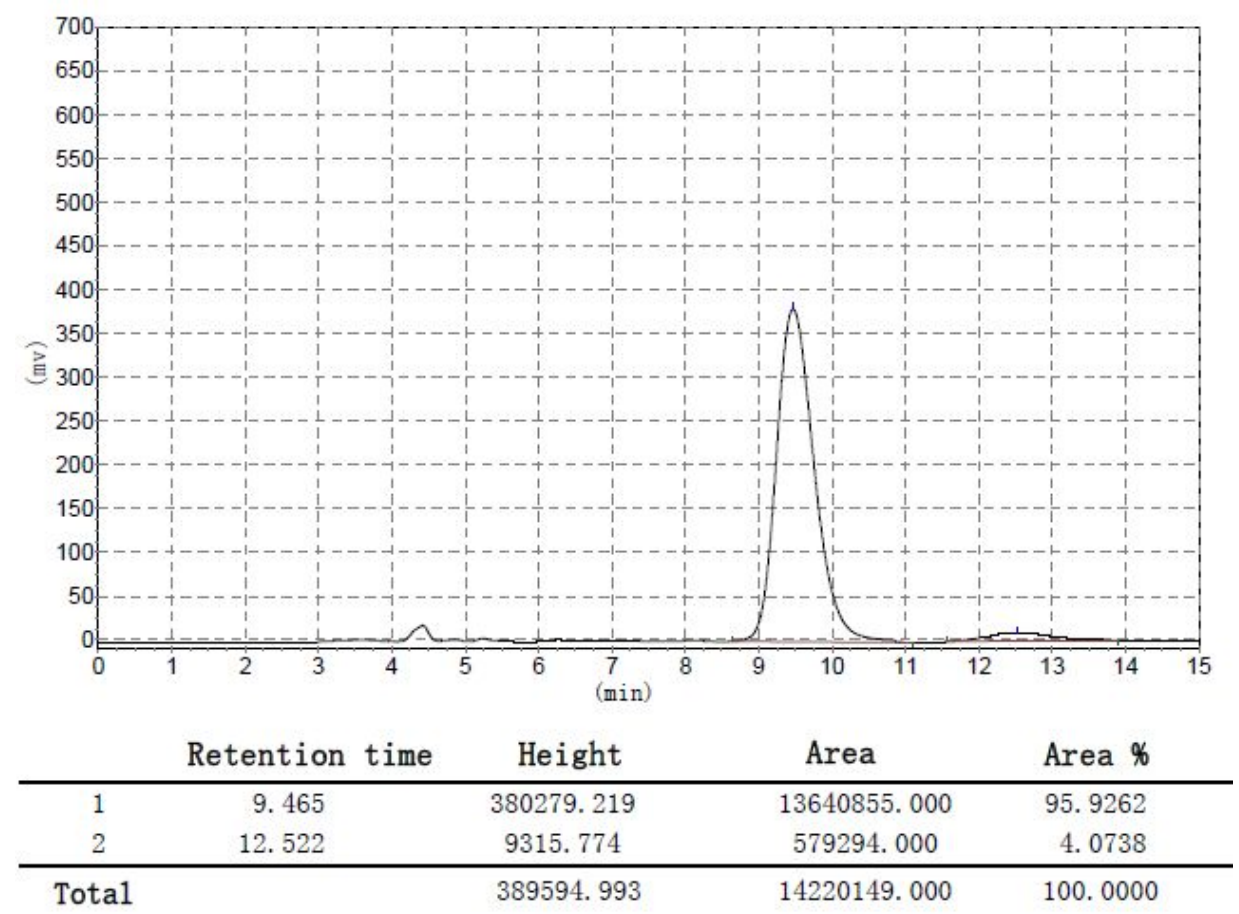




\section{$8 y$ (Table 4, entry 8)}<smiles>COc1oc(C2c3ccc4ccccc4c3CCN2NC(F)(F)F)nc1-c1ccccc1</smiles>

\section{Racemic}

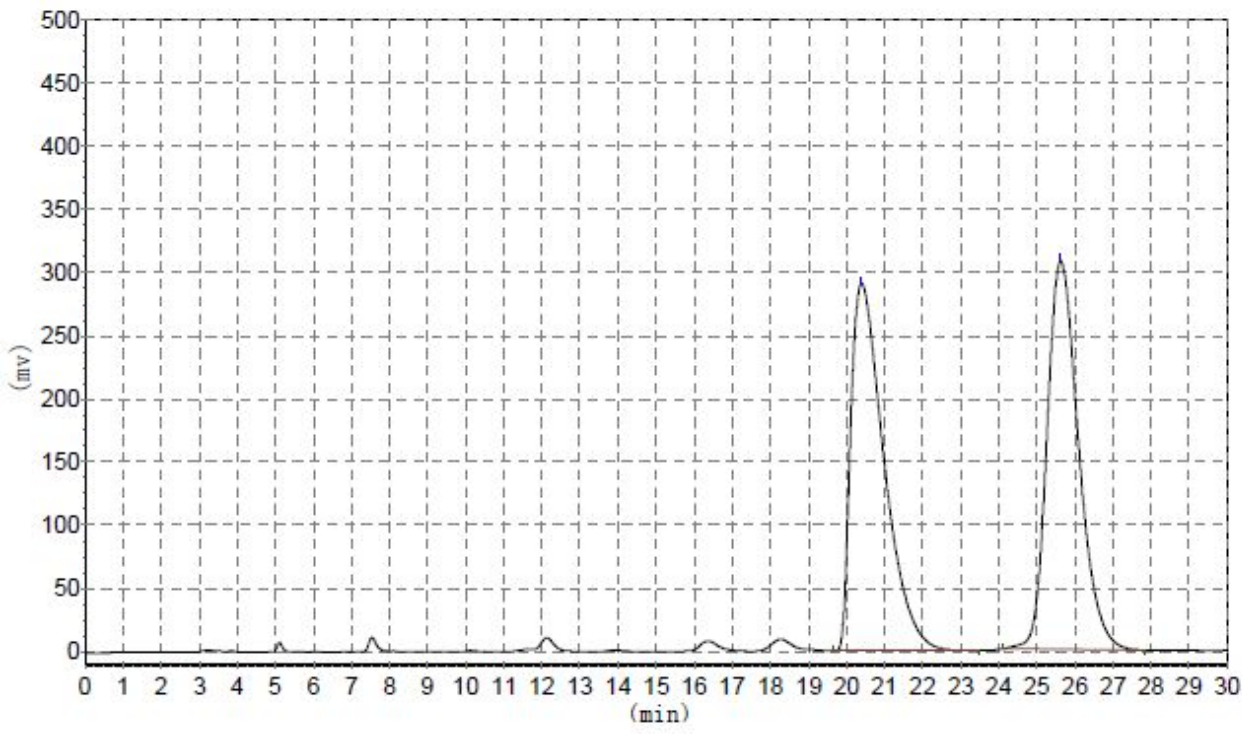

\begin{tabular}{ccccc} 
& Retention time & Height & Area & Area \% \\
\hline 1 & 20.397 & 290884.188 & 17650156.000 & 49.8976 \\
2 & 25.628 & 307204.250 & 17722610.000 & 50.1024 \\
\hline Total & & 598088.438 & 35372766.000 & 100.0000
\end{tabular}

\section{Chiral}

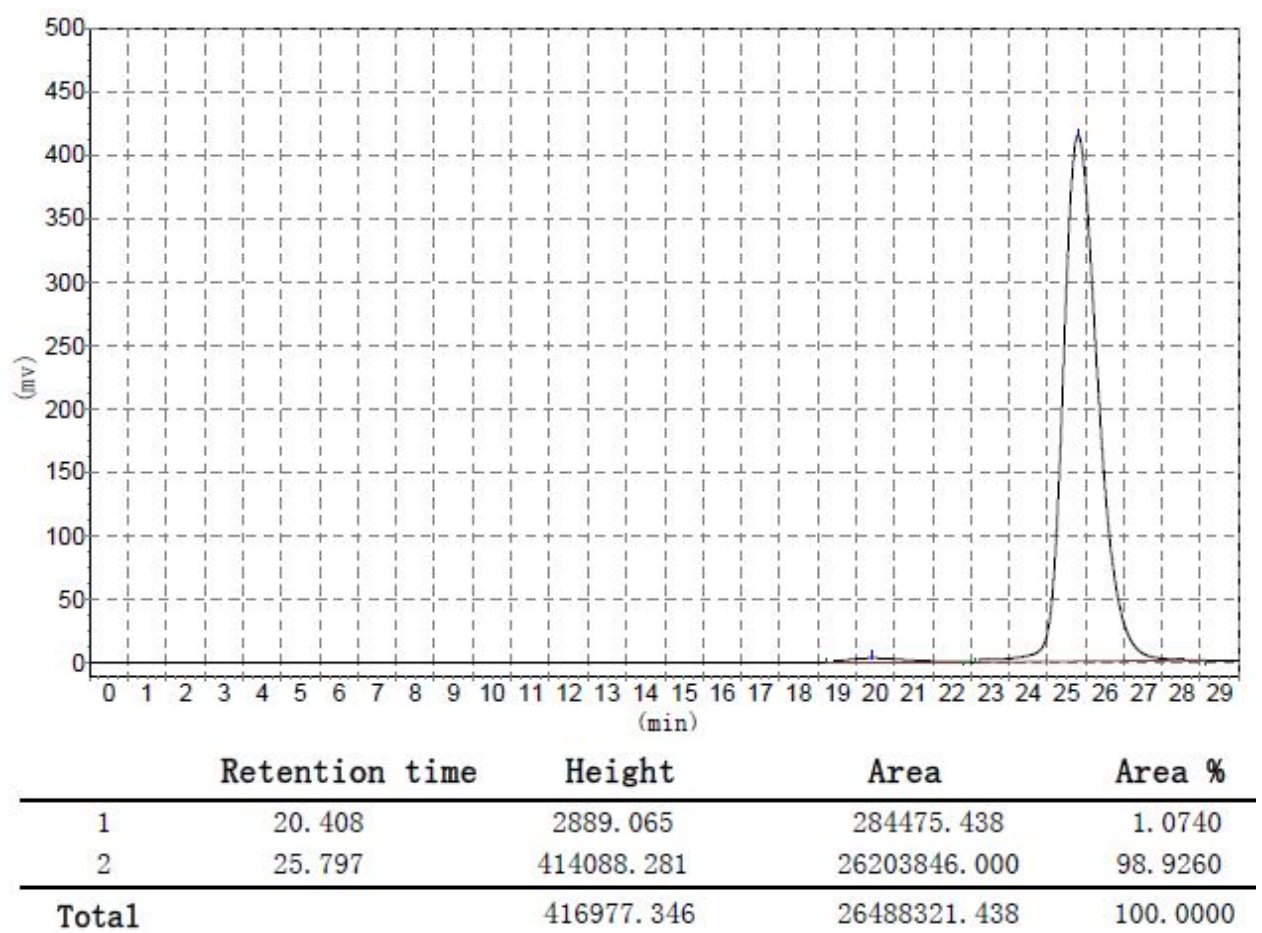


3. Copies of NMR Spectra for the Compounds 7 a and 8

${ }^{1} \mathrm{H}$ NMR of $7 \mathbf{a}$
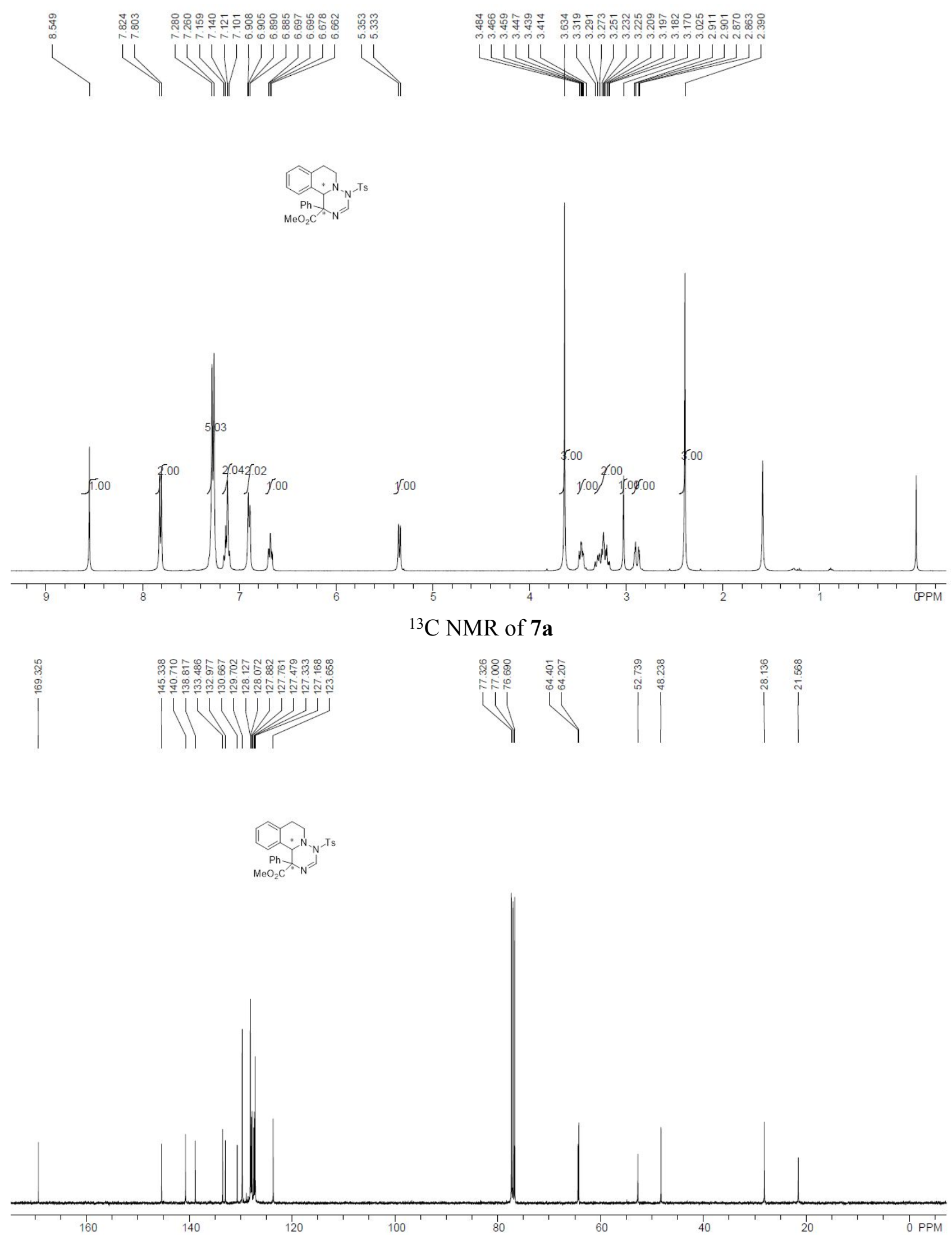
${ }^{1} \mathrm{H}$ NMR of $\mathbf{8 a}$
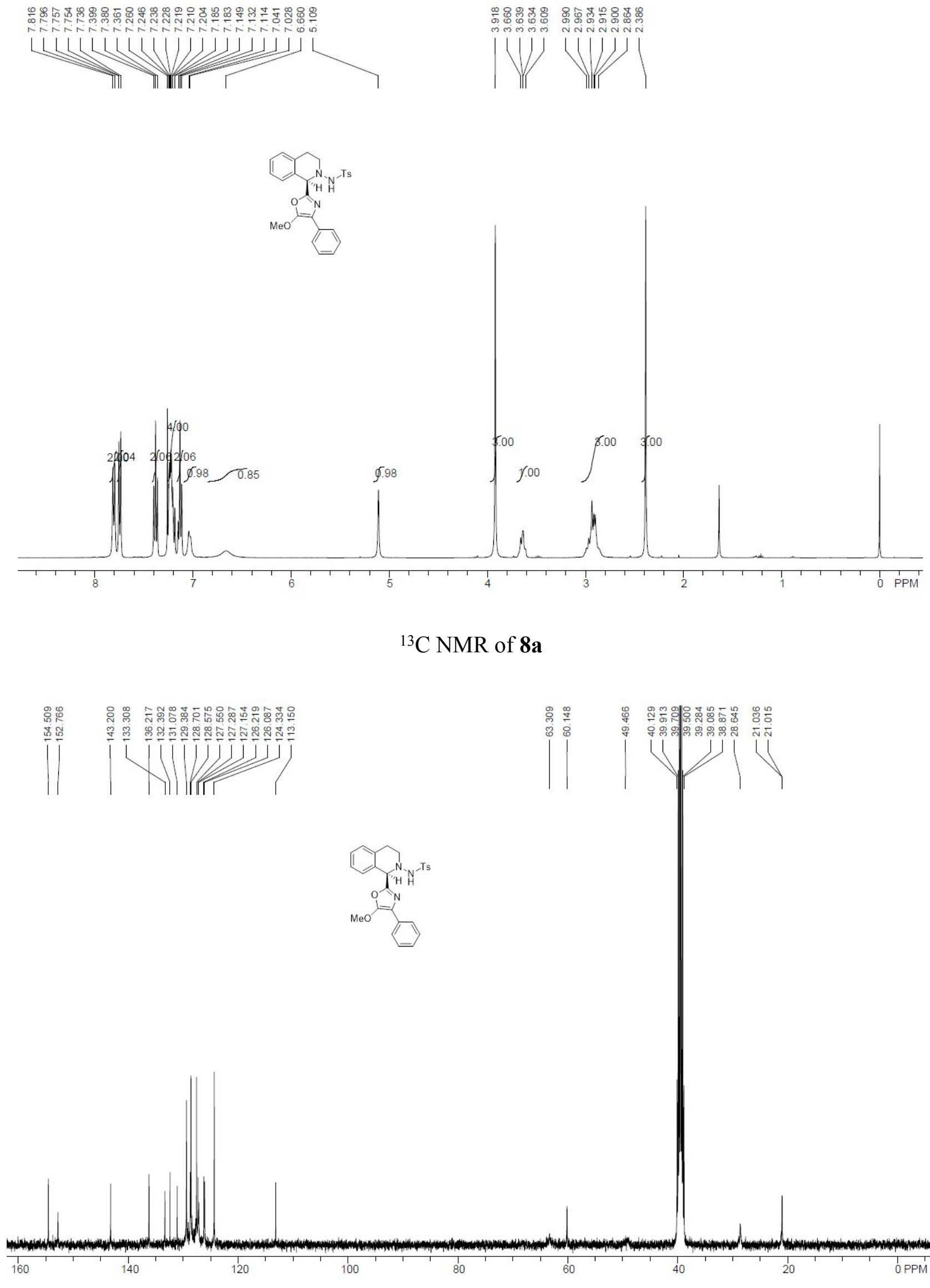
${ }^{13} \mathrm{C}$ NMR of $\mathbf{8 a}\left(60^{\circ} \mathrm{C}, 600 \mathrm{M}\right)$
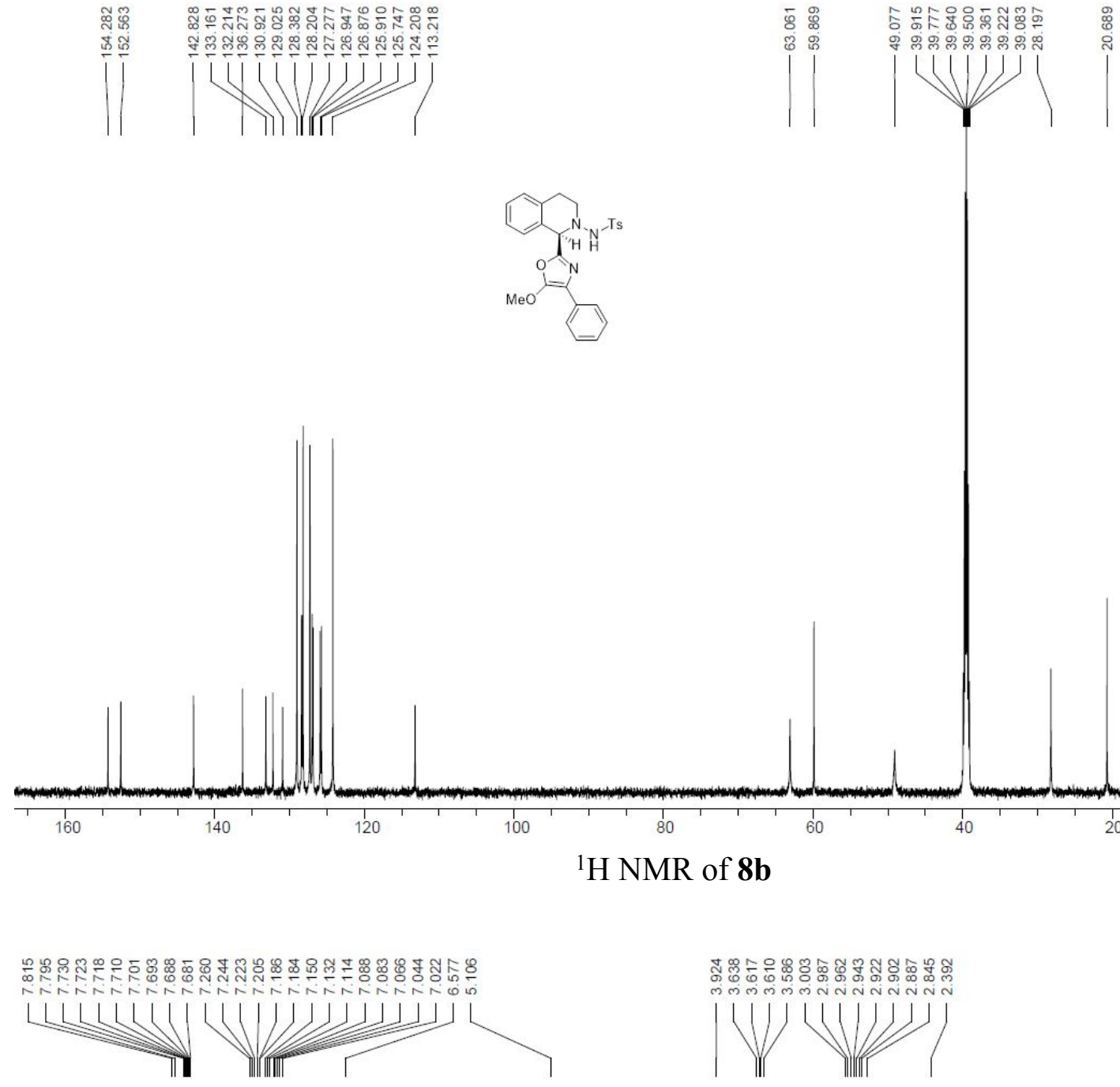

${ }^{1} \mathrm{H}$ NMR of $\mathbf{8 b}$

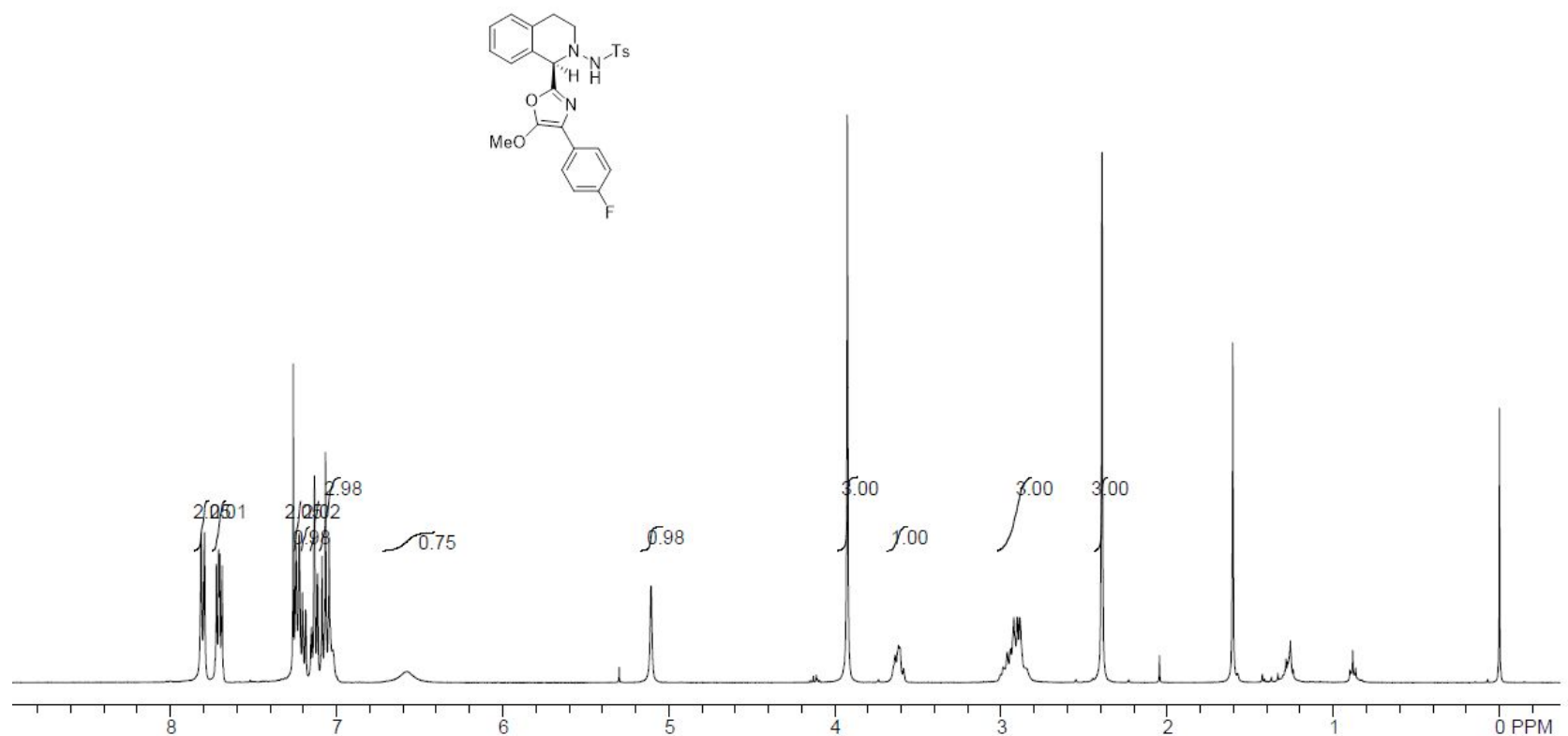


${ }^{13} \mathrm{C}$ NMR of $\mathbf{8 b}$

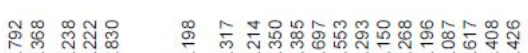

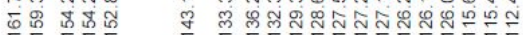

$\left.\left.\right|^{1} \int^{1}\right|^{2}$

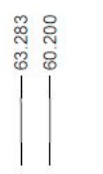

(1)
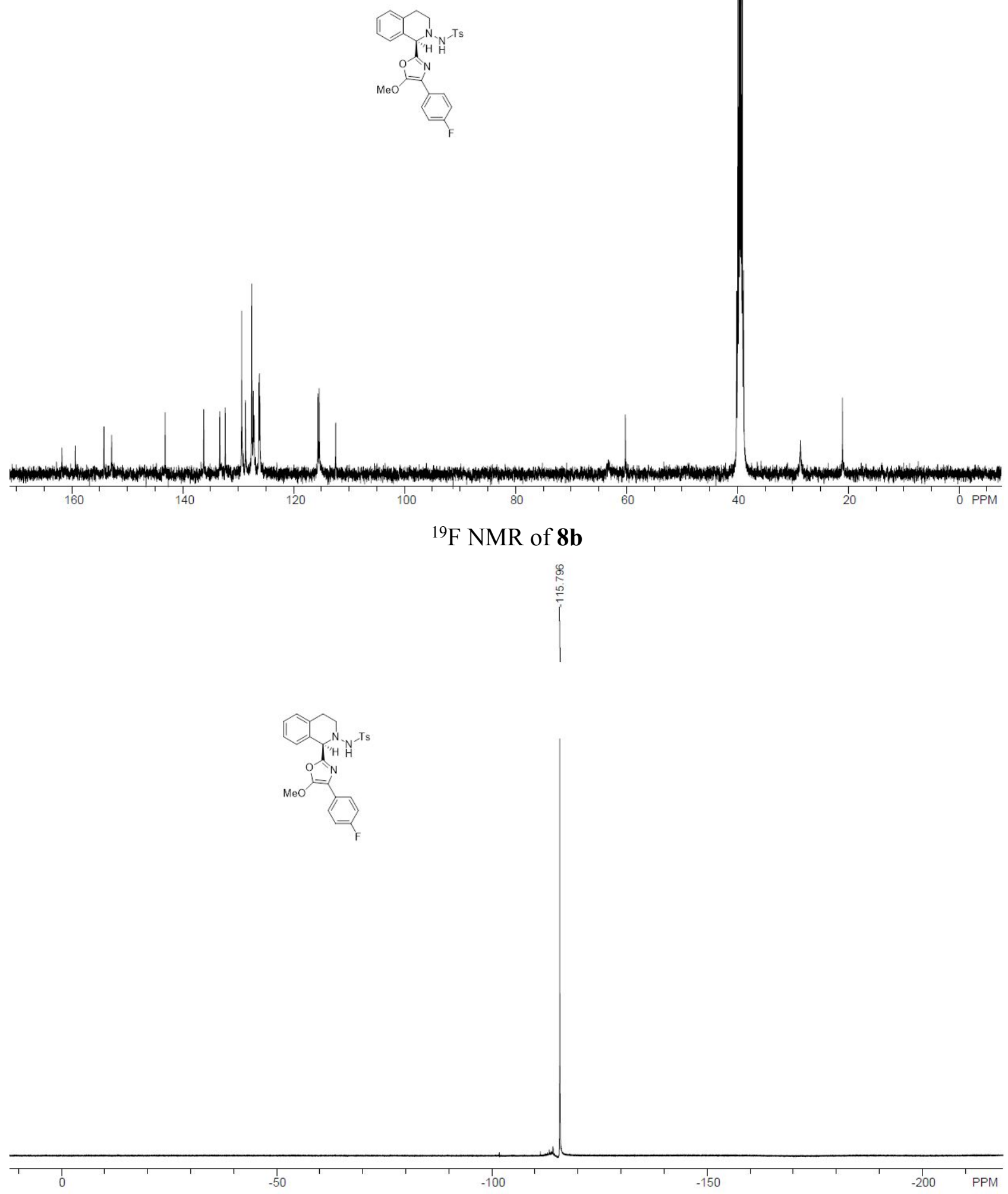

60

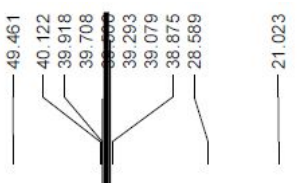


${ }^{1} \mathrm{H}$ NMR of $\mathbf{8 c}$

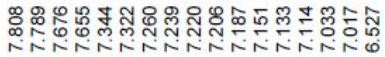
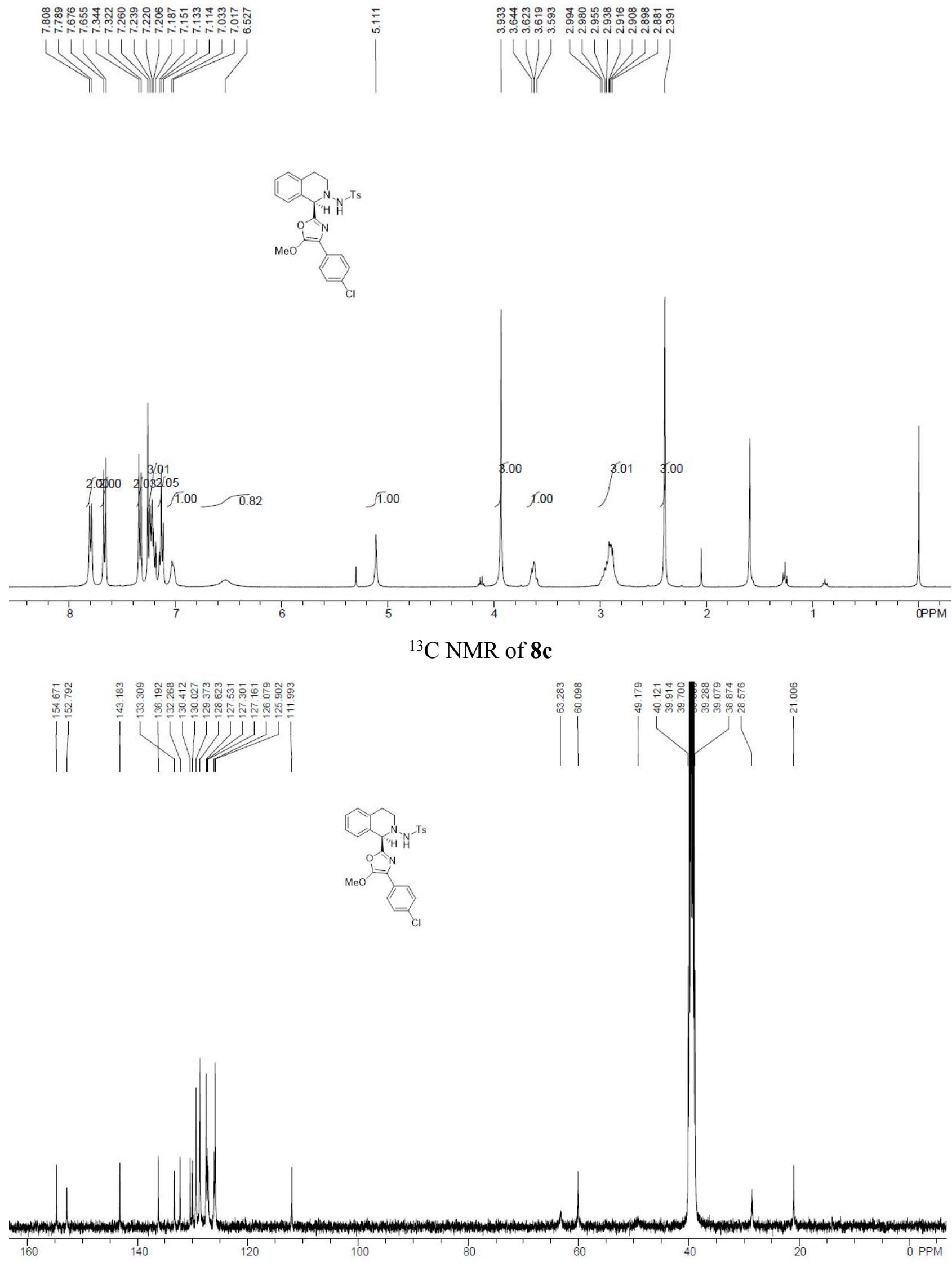

${ }^{13} \mathrm{C}$ NMR of $8 \mathrm{c}$ 
${ }^{1} \mathrm{H}$ NMR of $\mathbf{8 d}$
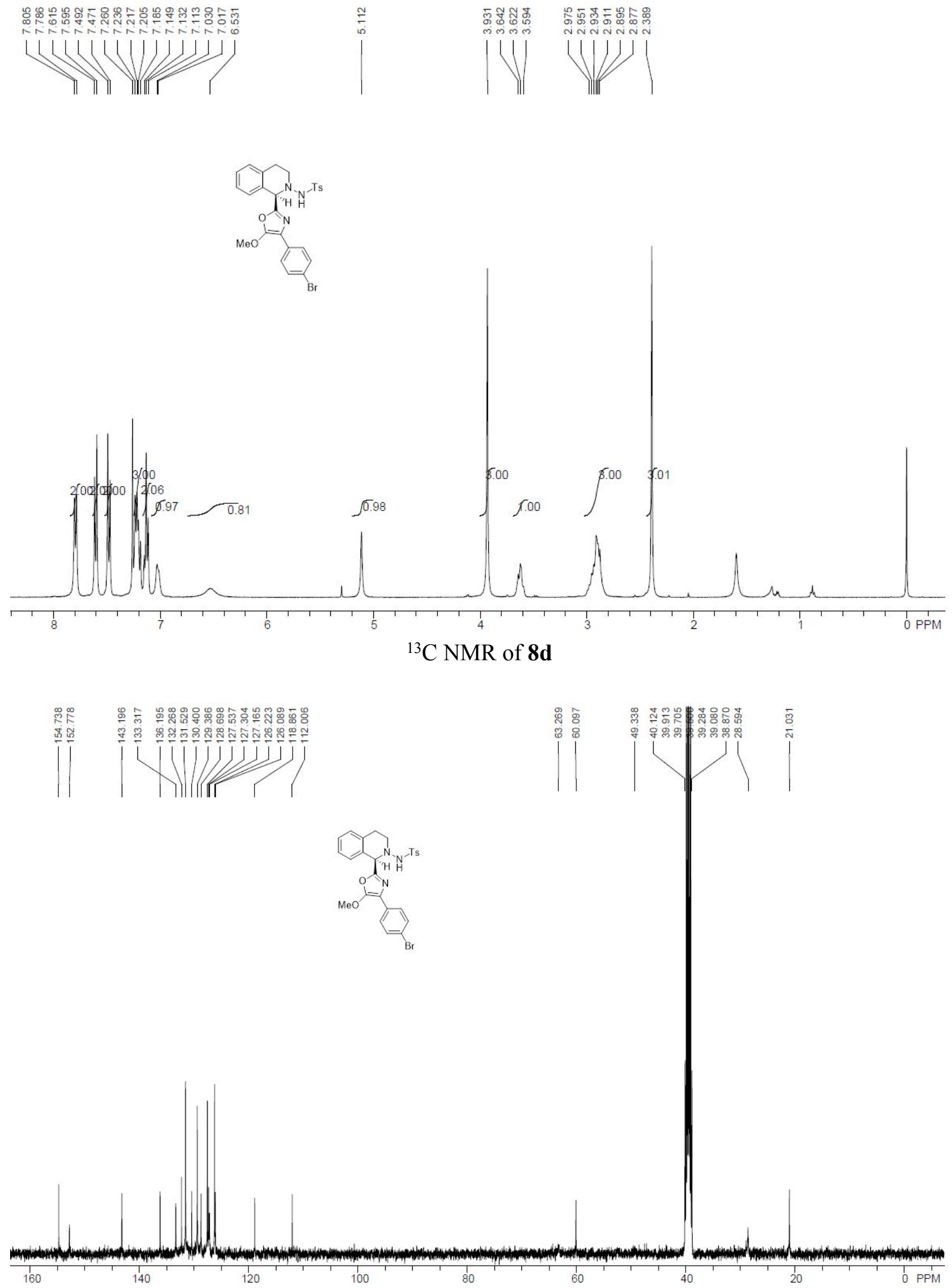
${ }^{1} \mathrm{H}$ NMR of $8 \mathbf{e}$
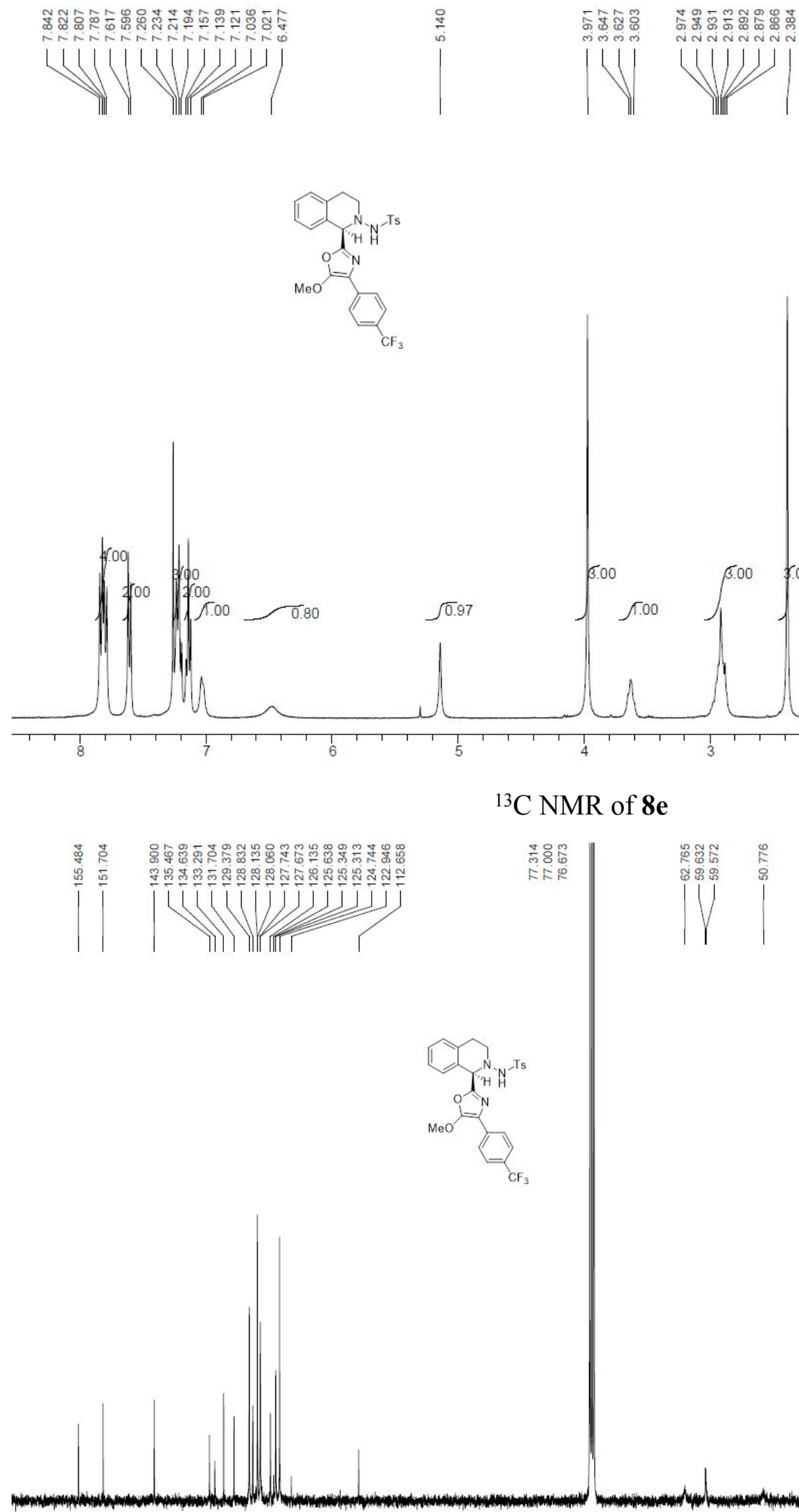

160

140

120 
${ }^{19} \mathrm{~F}$ NMR of $8 \mathbf{e}$

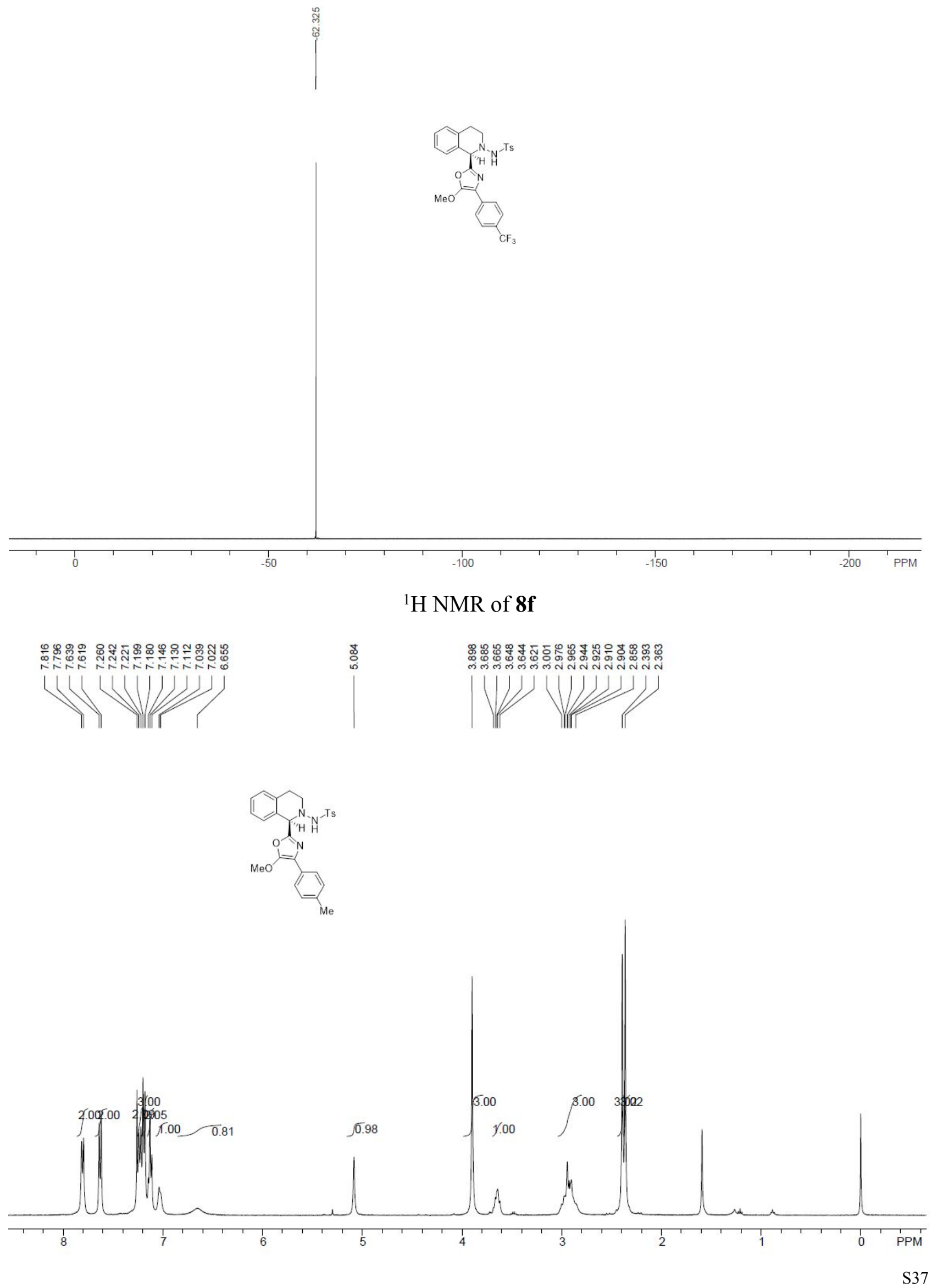


${ }^{13} \mathrm{C}$ NMR of $\mathbf{8 f}$
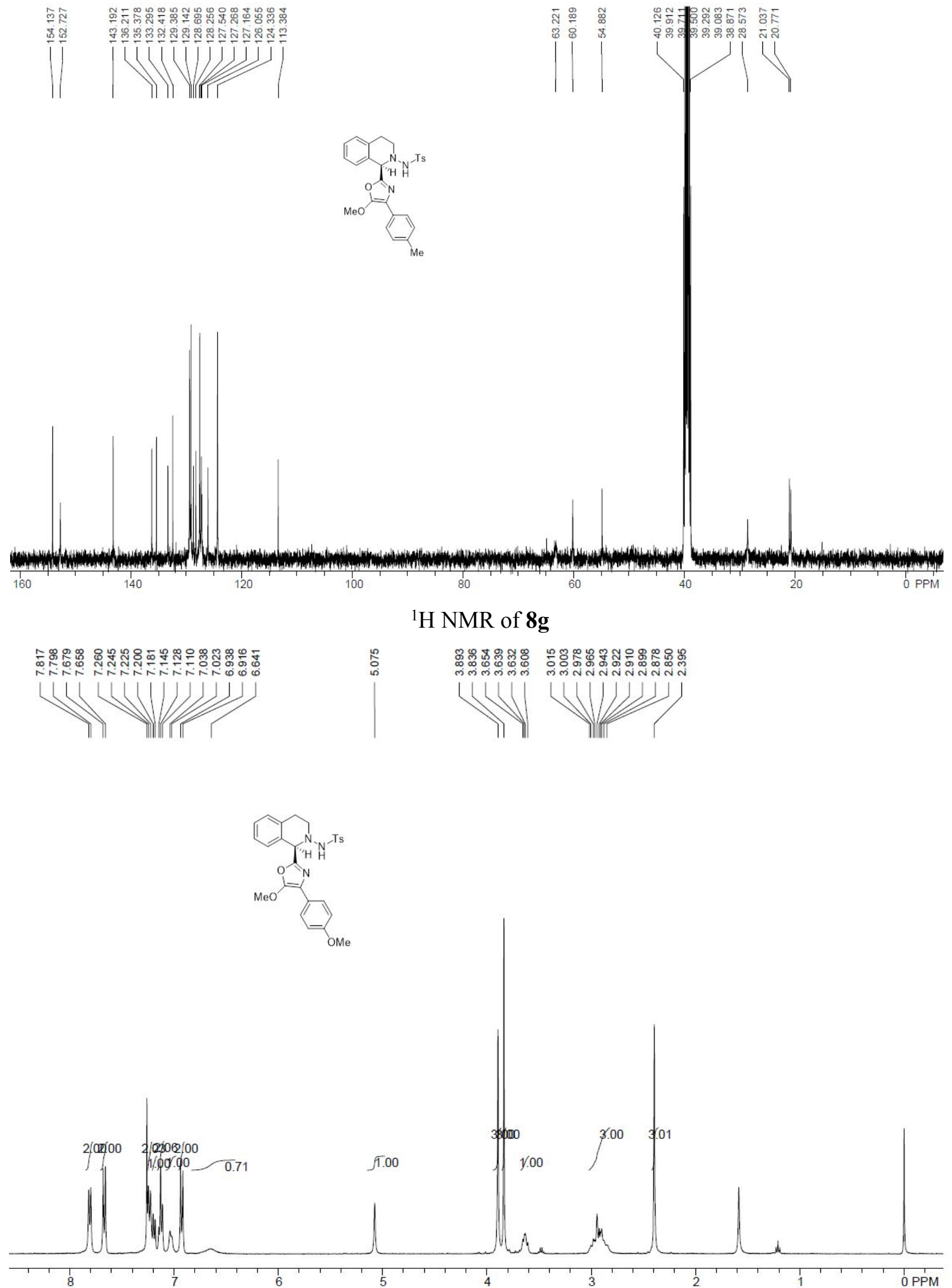
${ }^{13} \mathrm{C}$ NMR of $8 \mathrm{~g}$
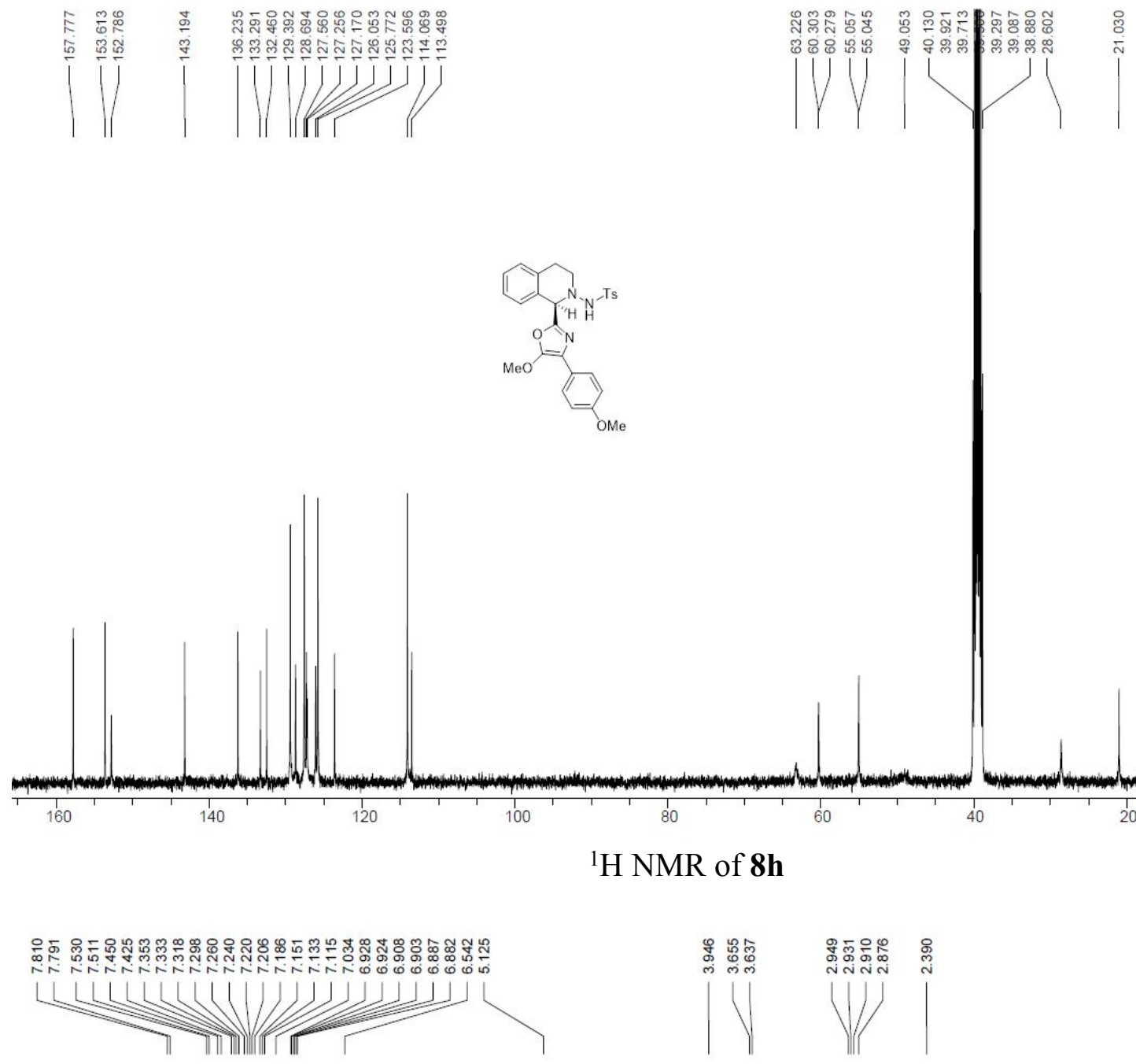

${ }^{1} \mathrm{H}$ NMR of $\mathbf{8 h}$

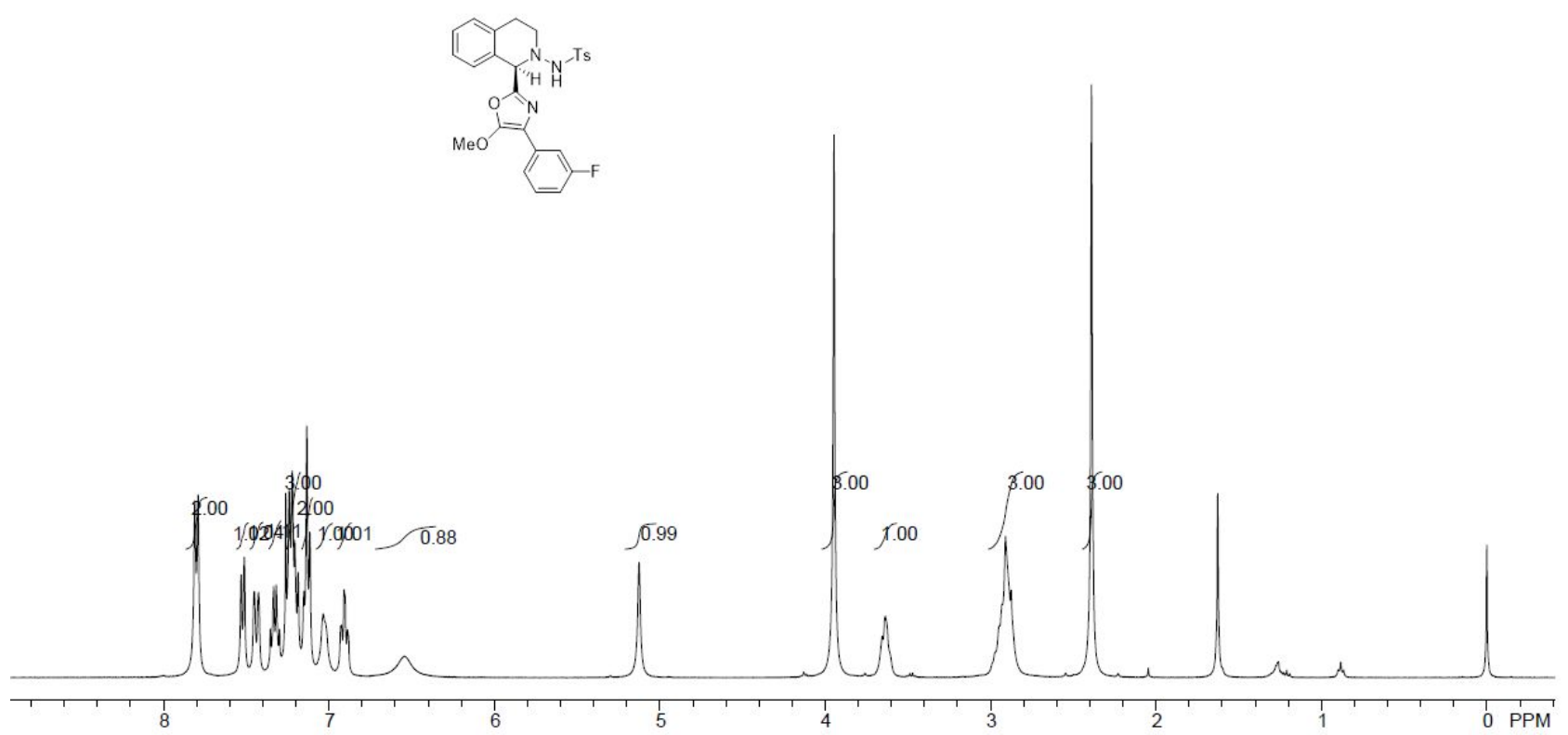


${ }^{13} \mathrm{C}$ NMR of $\mathbf{8 h}$
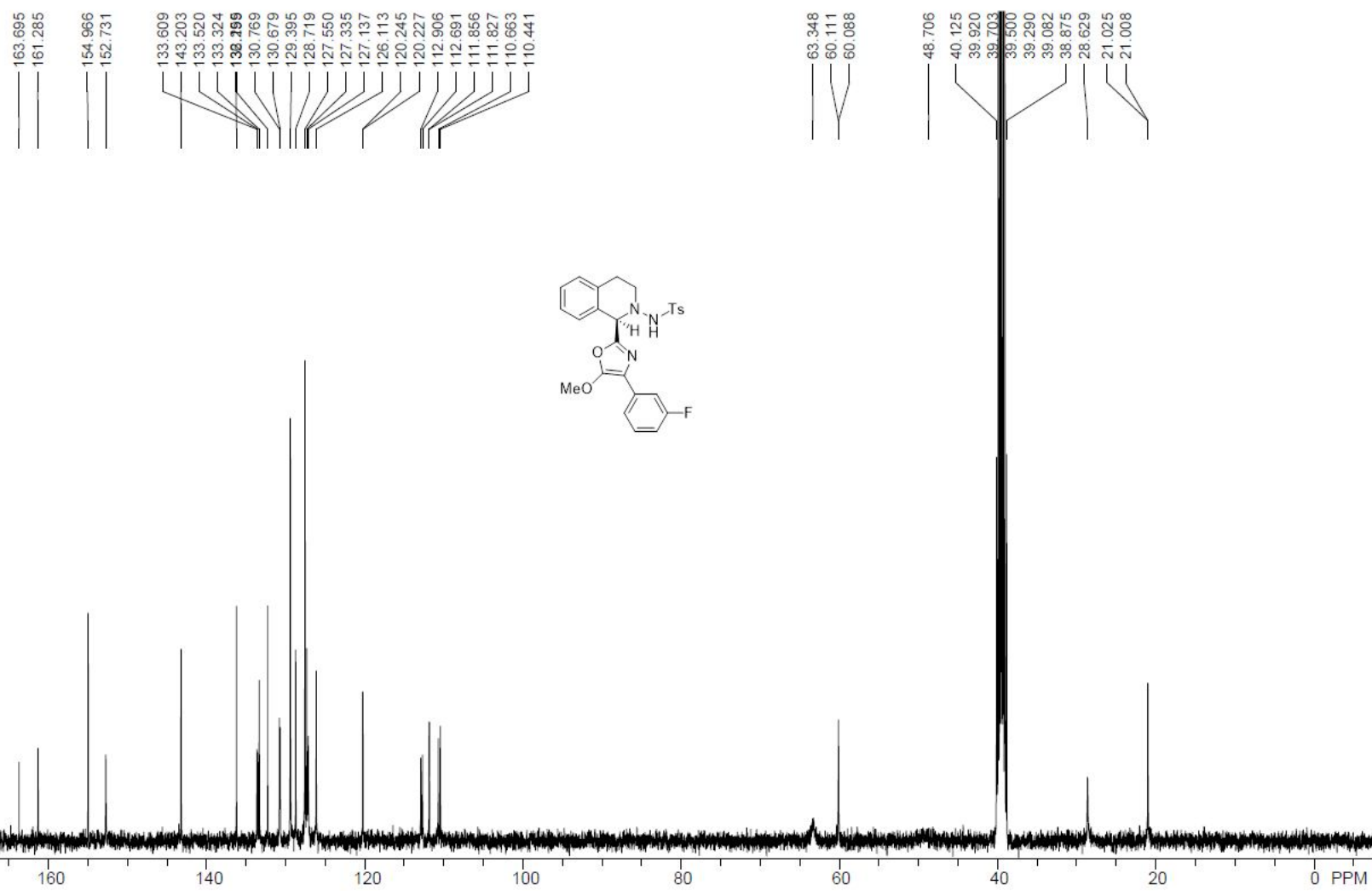

${ }^{19} \mathrm{~F}$ NMR of $\mathbf{8 h}$
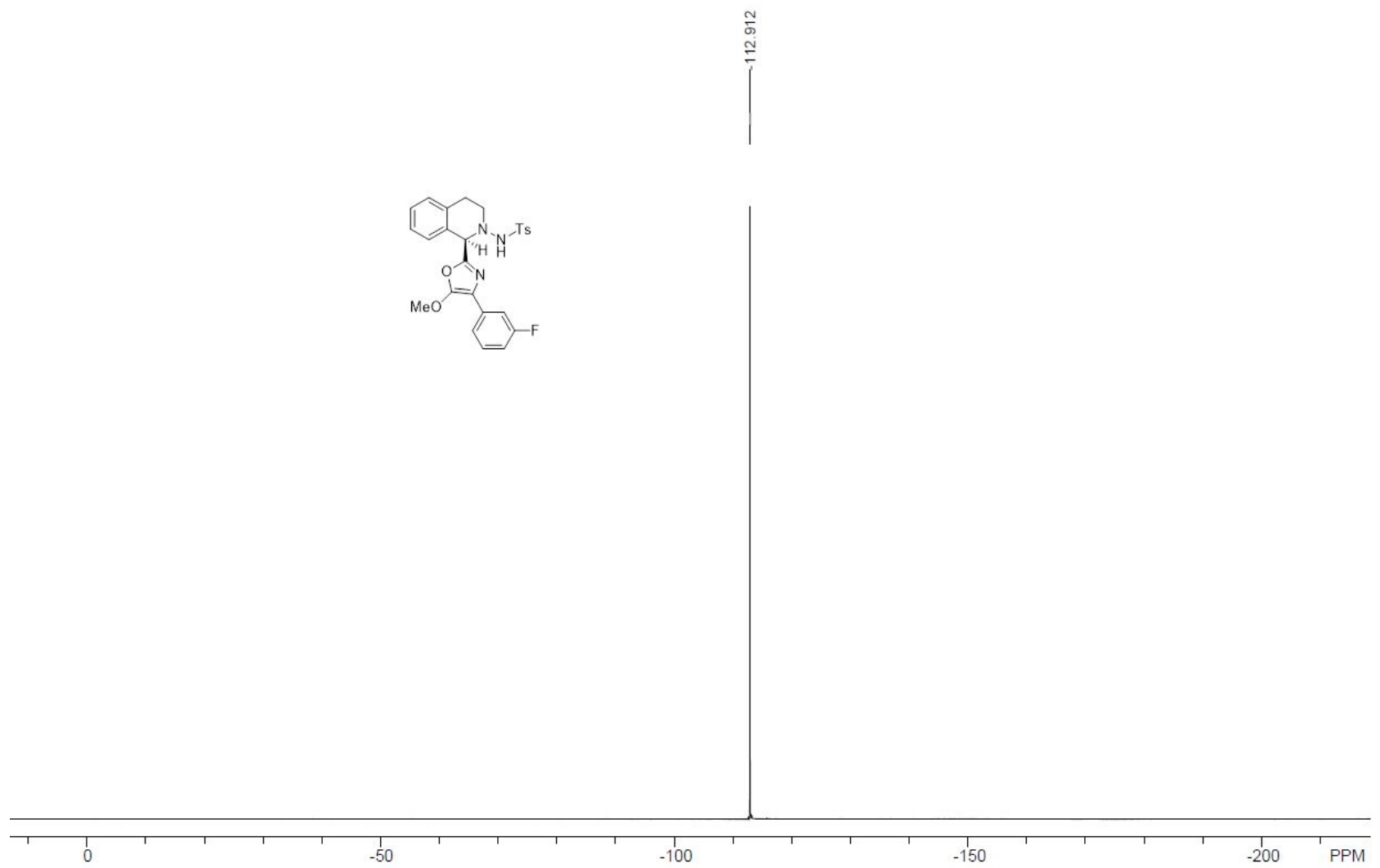
${ }^{1} \mathrm{H}$ NMR of $\mathbf{8 i}$
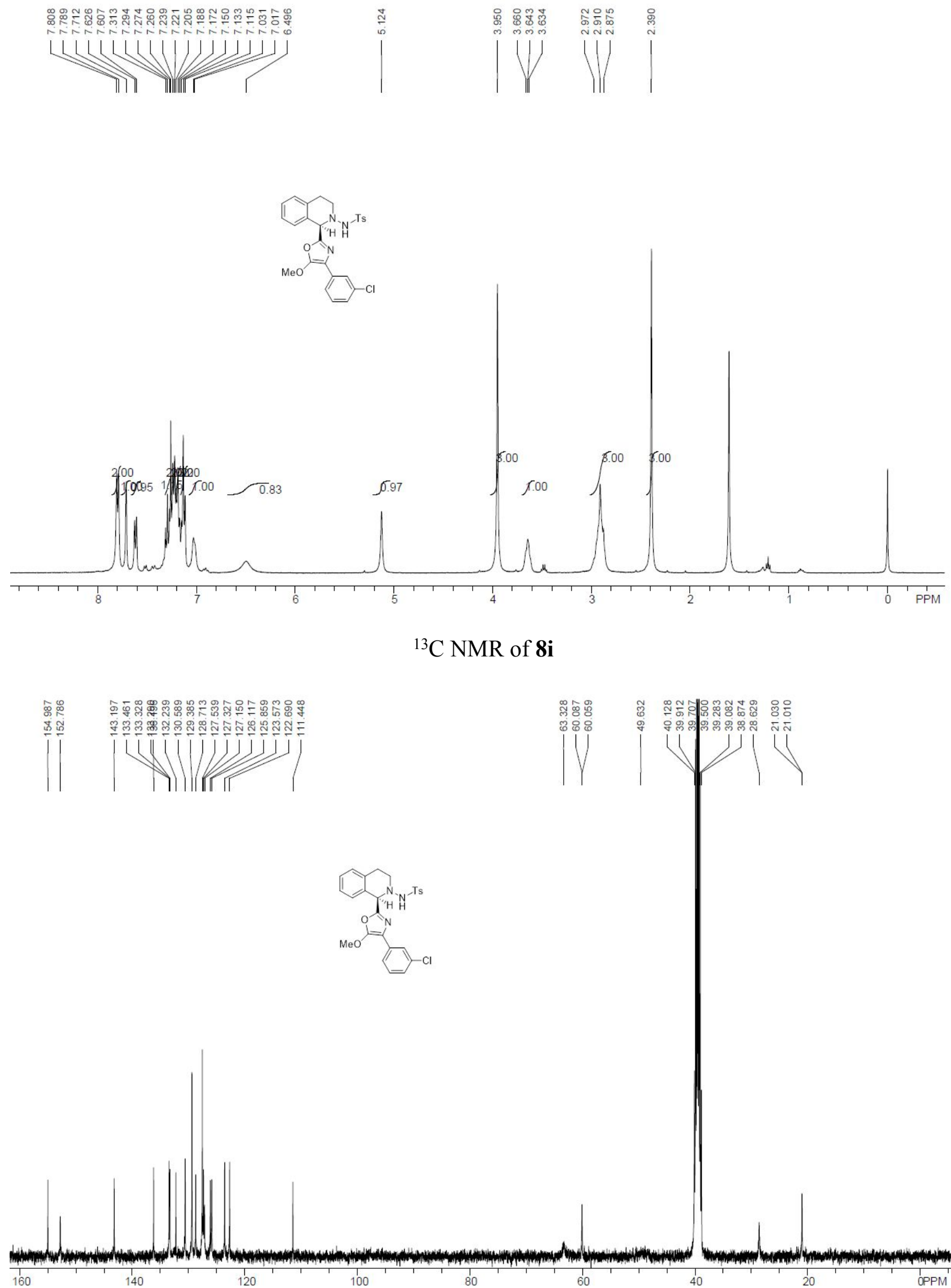
${ }^{1} \mathrm{H}$ NMR of $\mathbf{8 j}$
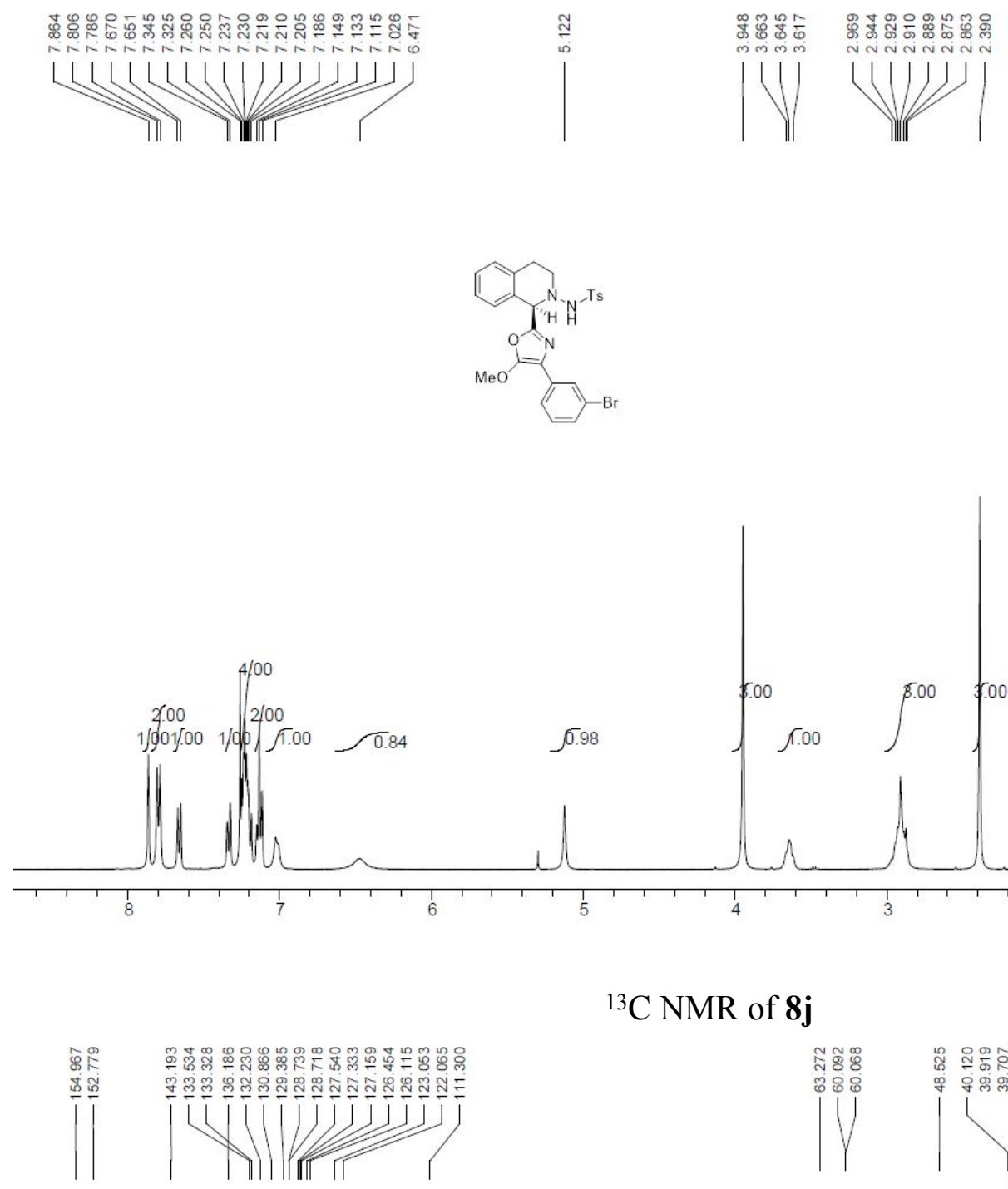

${ }^{13} \mathrm{C}$ NMR of $\mathbf{8 j}$
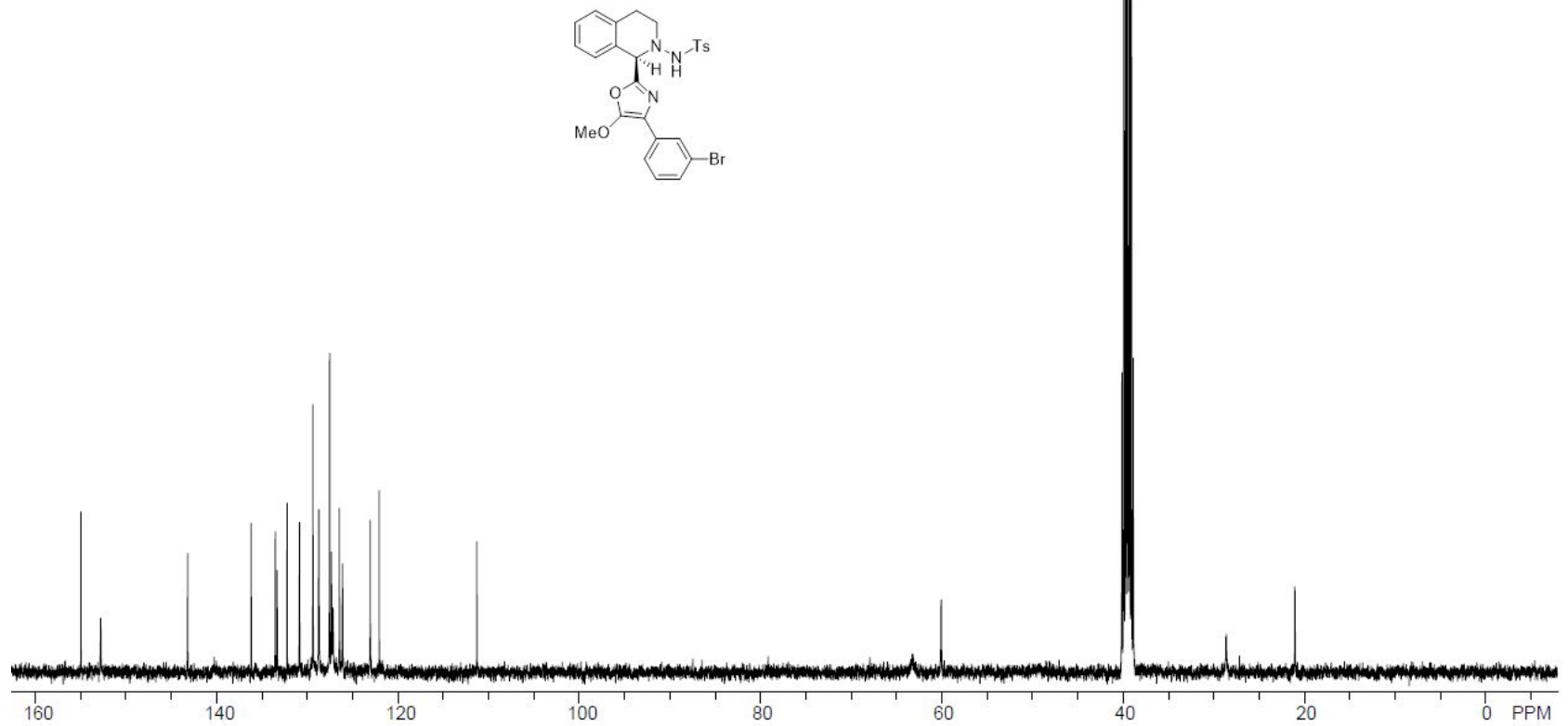
${ }^{1} \mathrm{H}$ NMR of $\mathbf{8 k}$
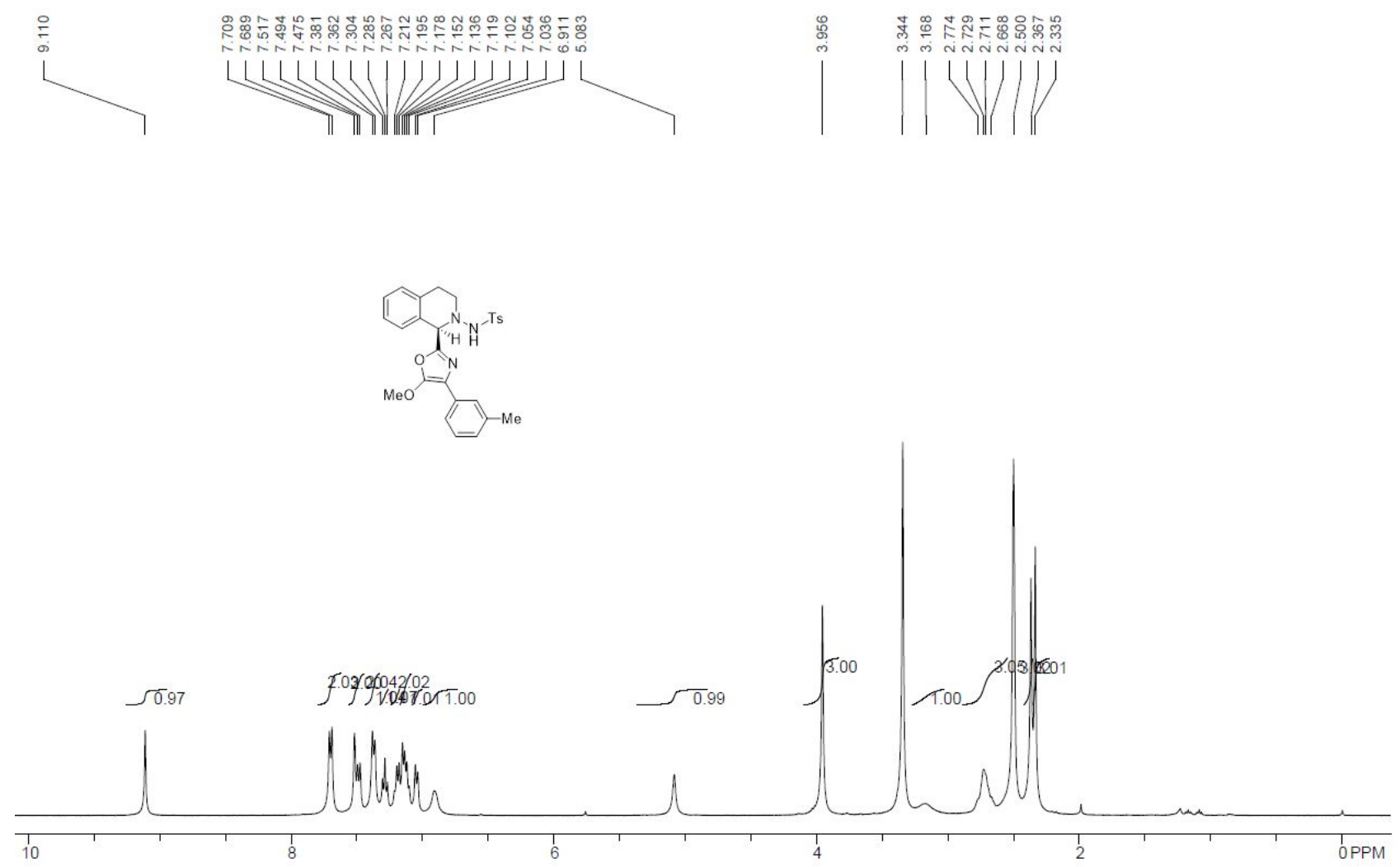

${ }^{13} \mathrm{C}$ NMR of $\mathbf{8 k}$
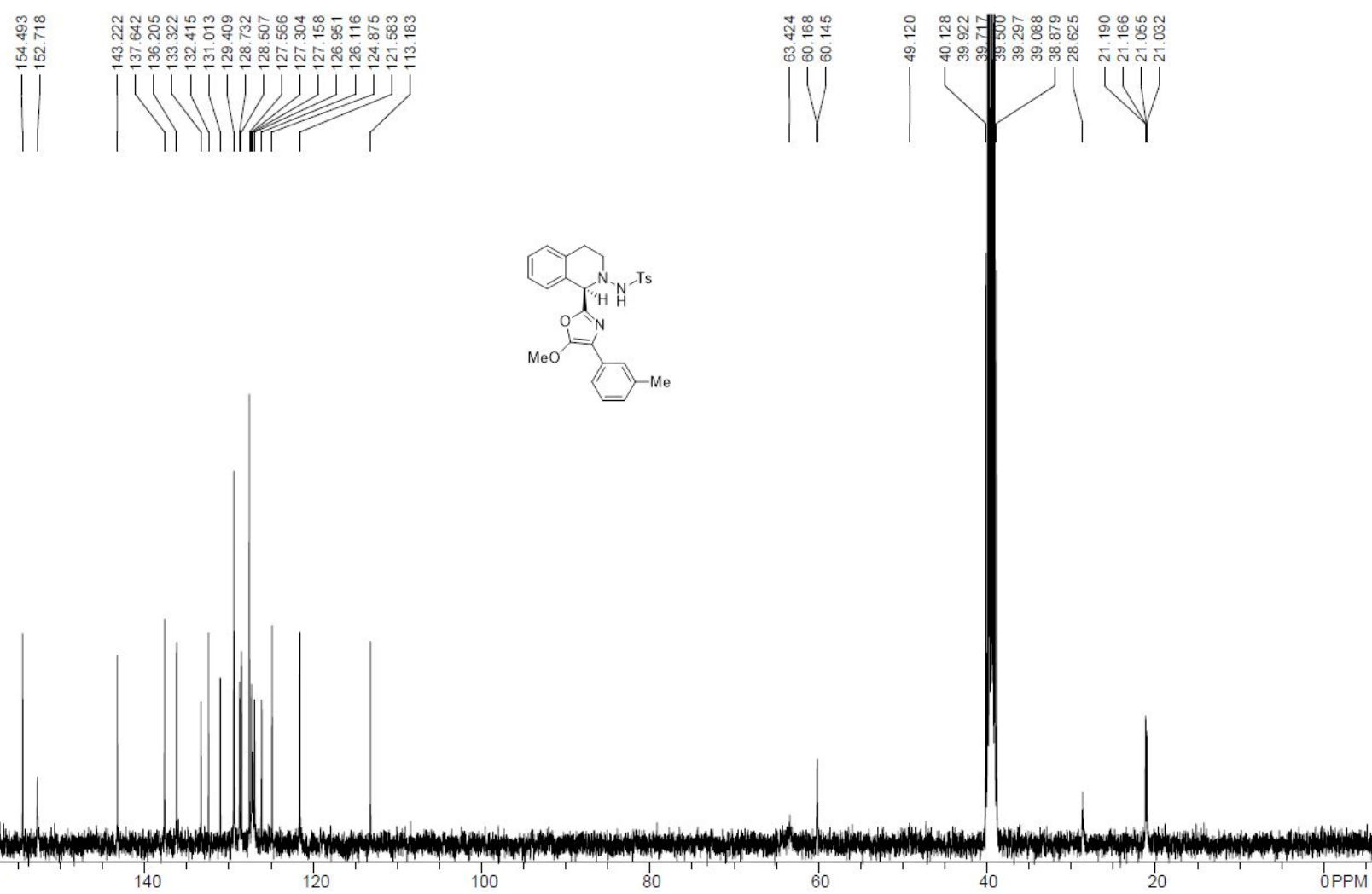
${ }^{1} \mathrm{H}$ NMR of $8 \mathbf{I}$

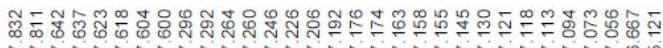

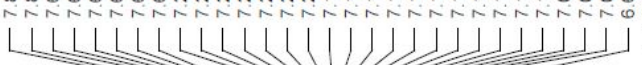

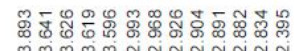

$14|1| 1|l| l \mid$
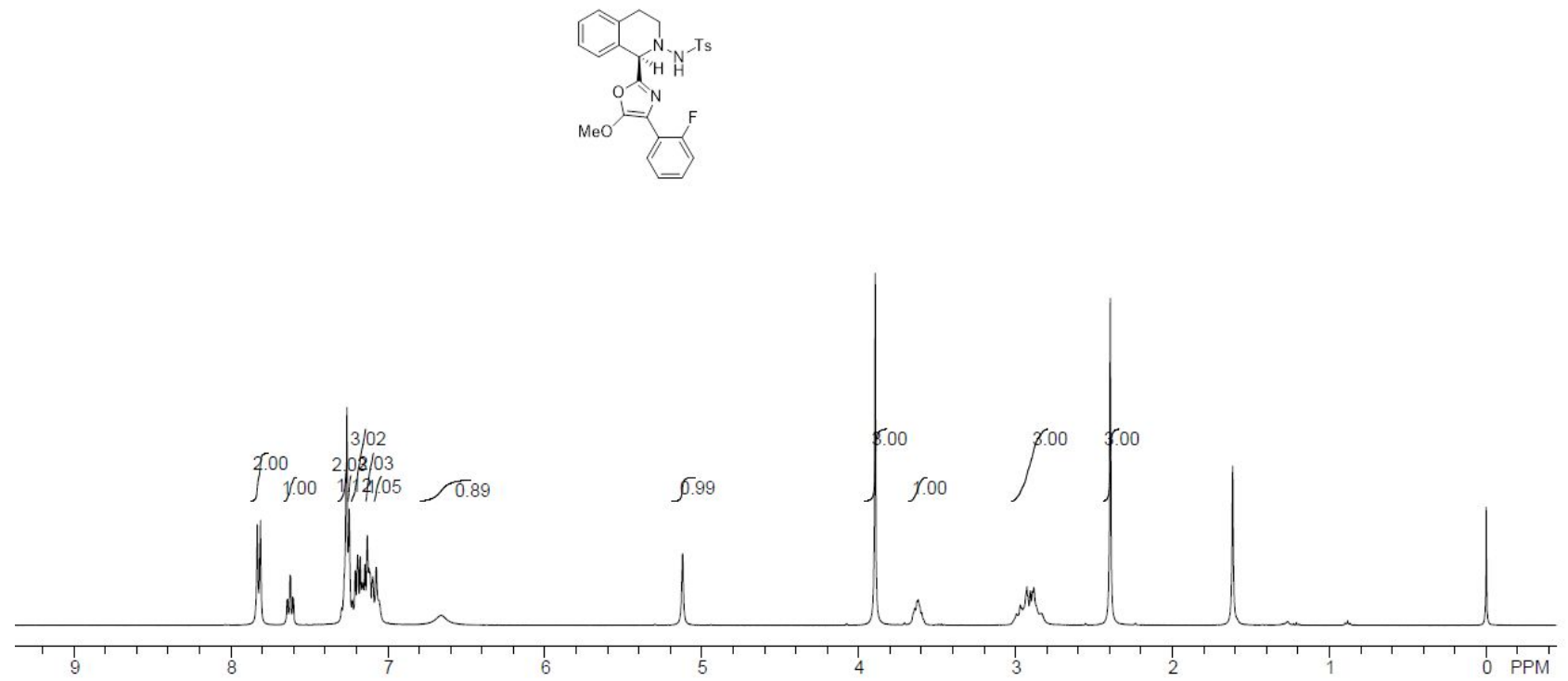

${ }^{13} \mathrm{C}$ NMR of 81
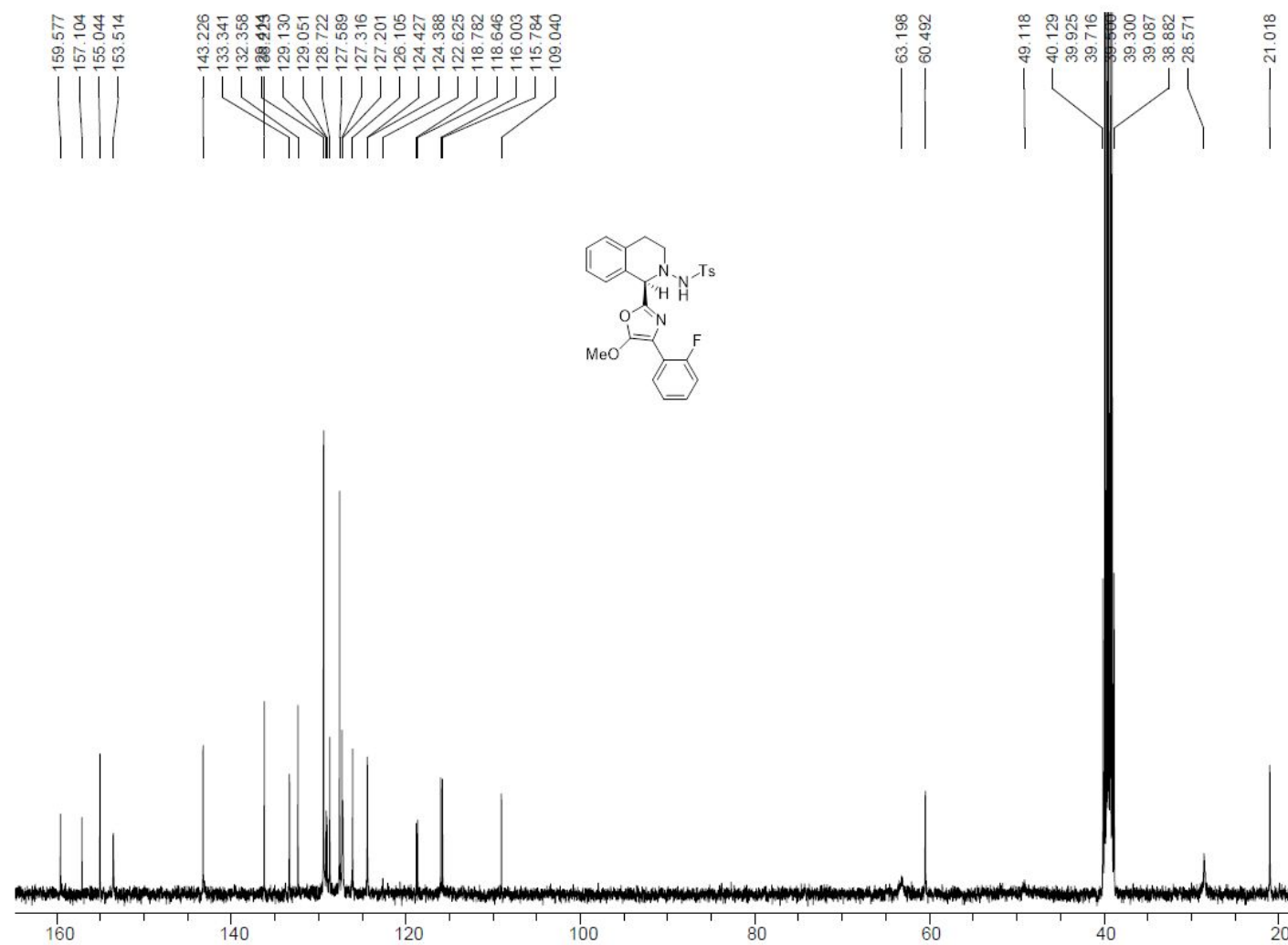

100

80

40

20

$\begin{array}{ll}1 & \text { P'PM } \\ 0 & \text { PPM }\end{array}$ 
${ }^{19} \mathrm{~F}$ NMR of $\mathbf{8 I}$

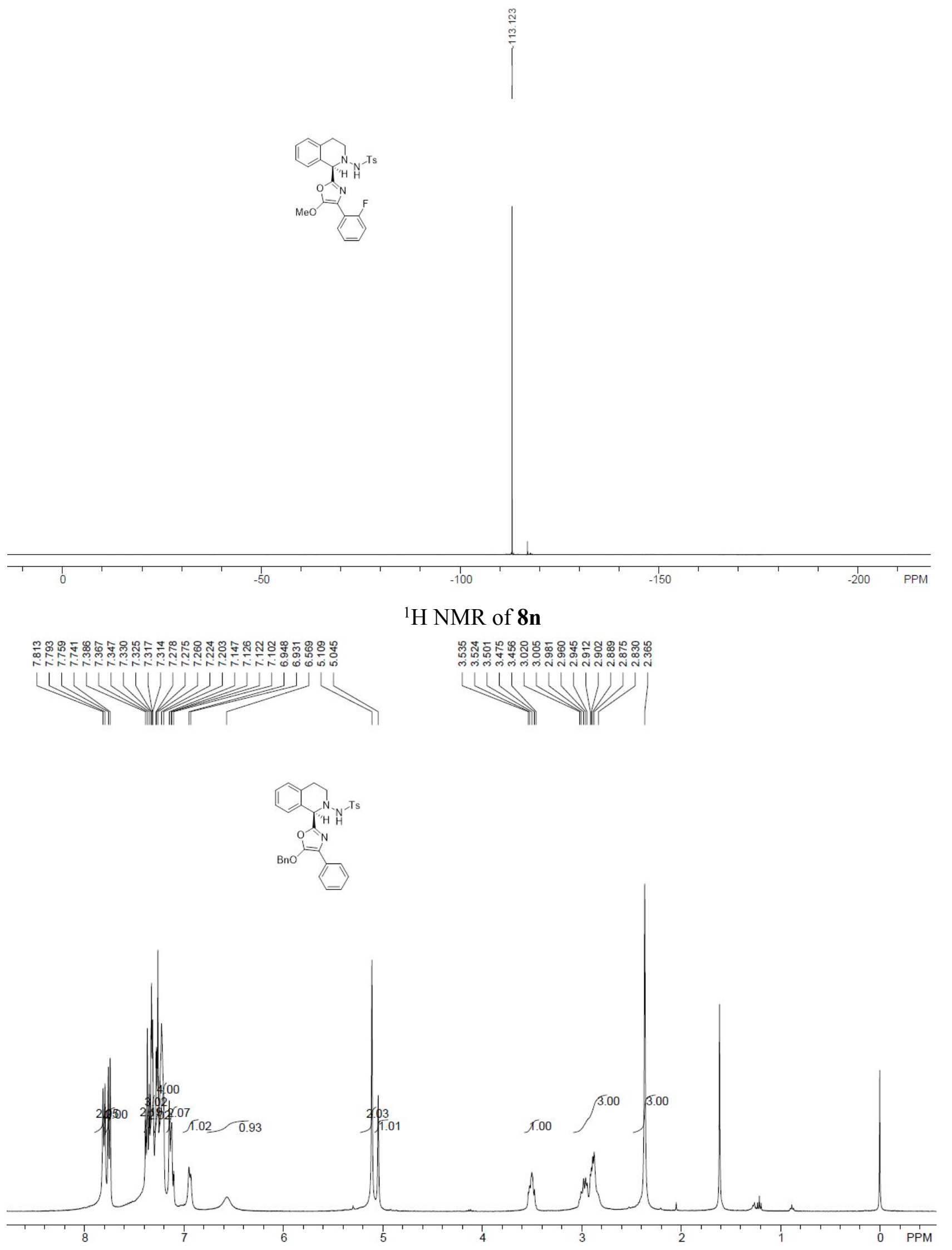


${ }^{13} \mathrm{C}$ NMR of $8 n$
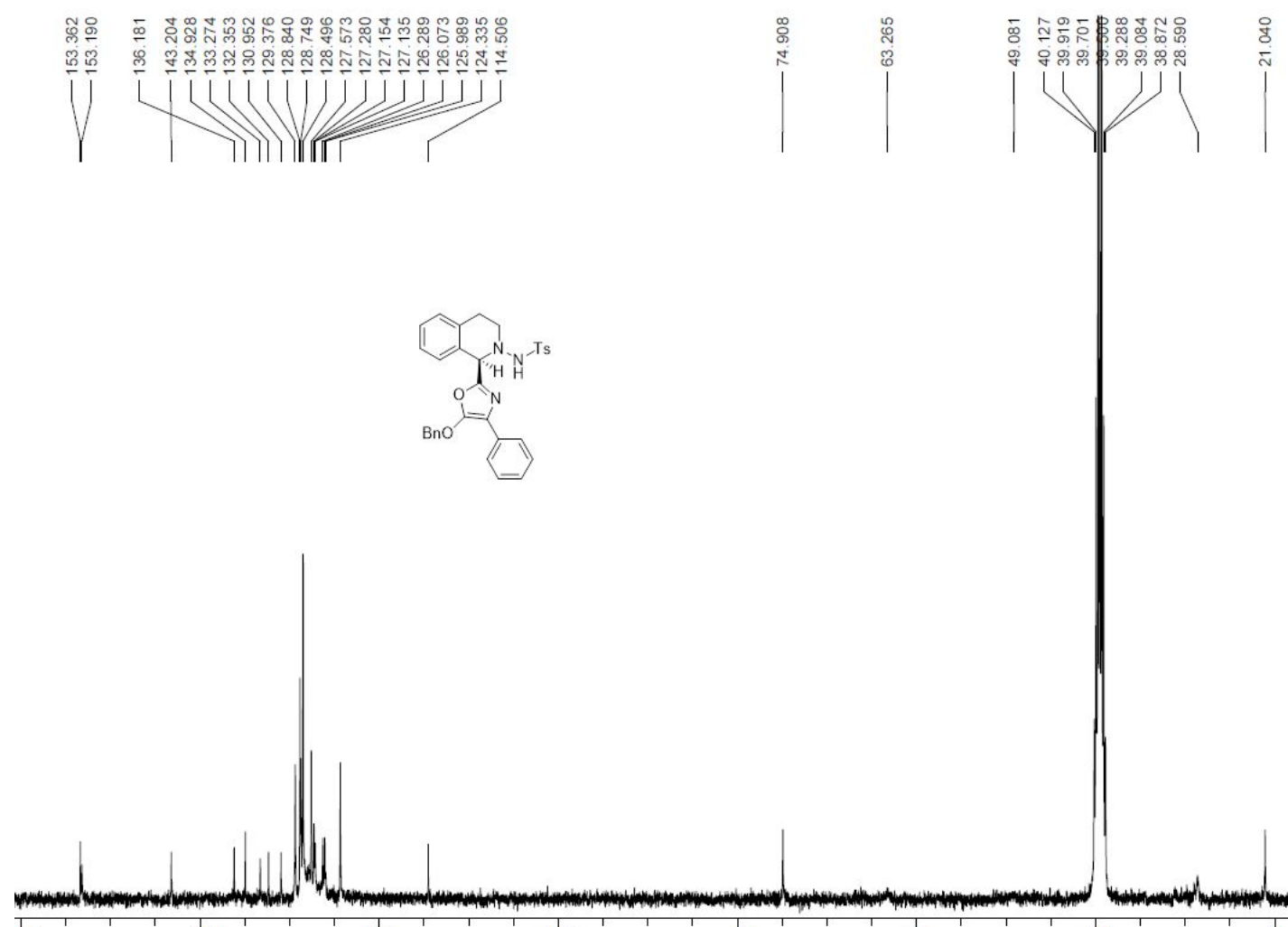

$\sqrt{60}$

120
120

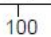

80

60

40

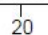

O PPM

${ }^{1} \mathrm{H}$ NMR of $\mathbf{8 0}$
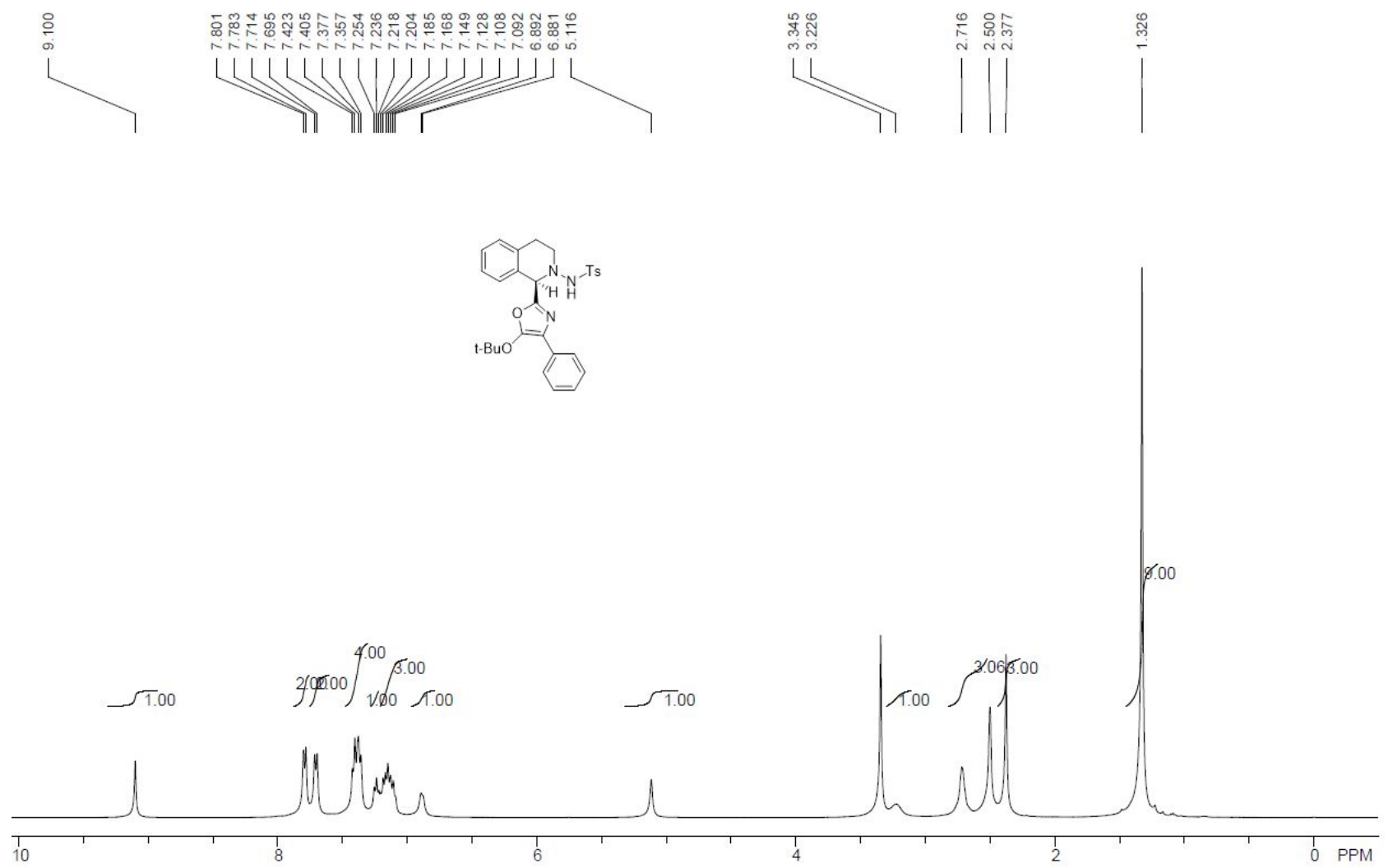
${ }^{13} \mathrm{C}$ NMR of 80
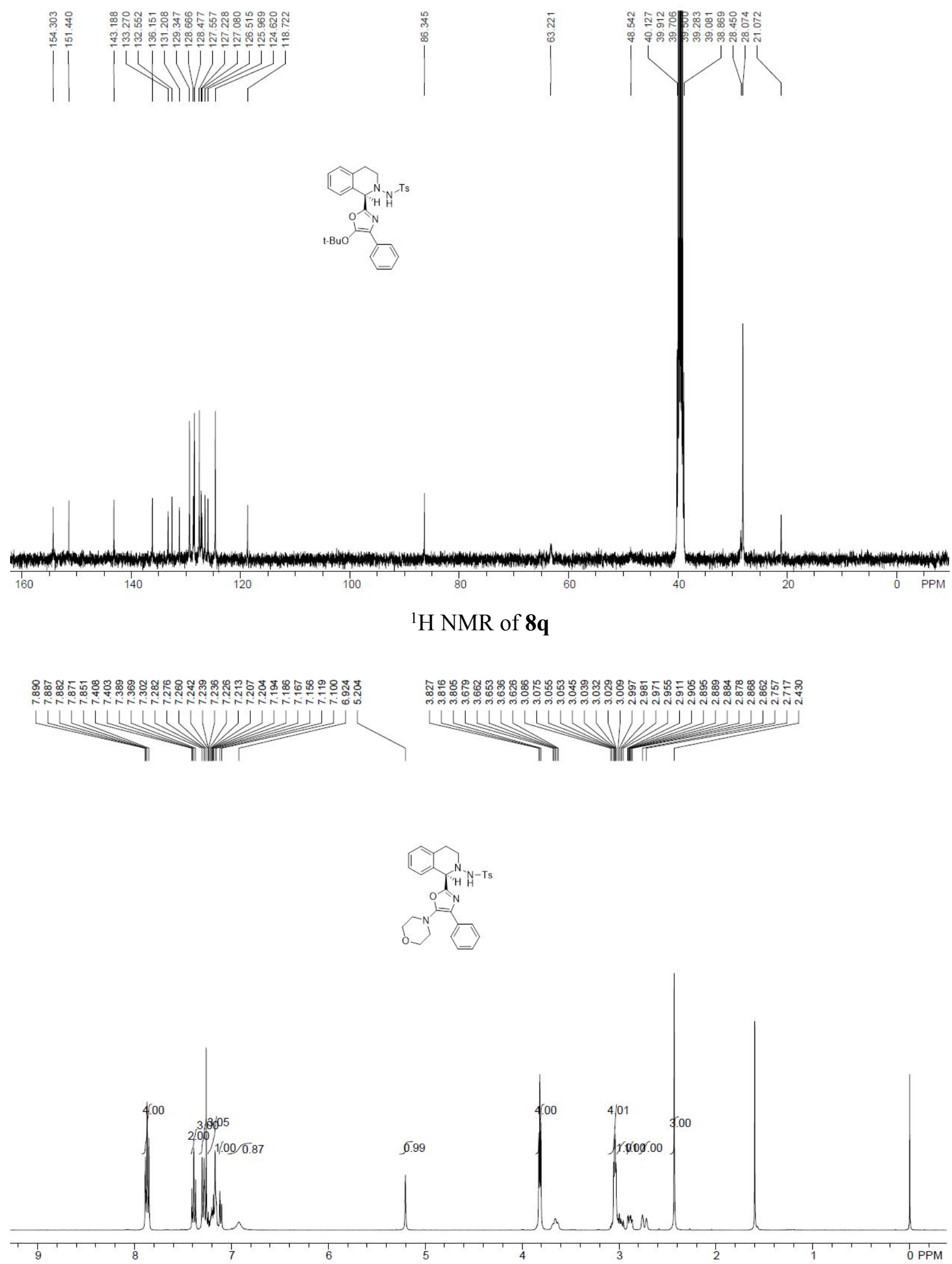
${ }^{13} \mathrm{C}$ NMR of $8 \mathbf{q}$

๓ สิ สิน

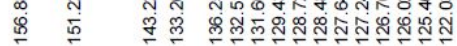

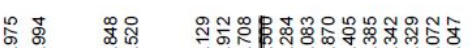

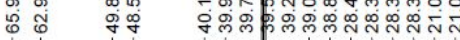

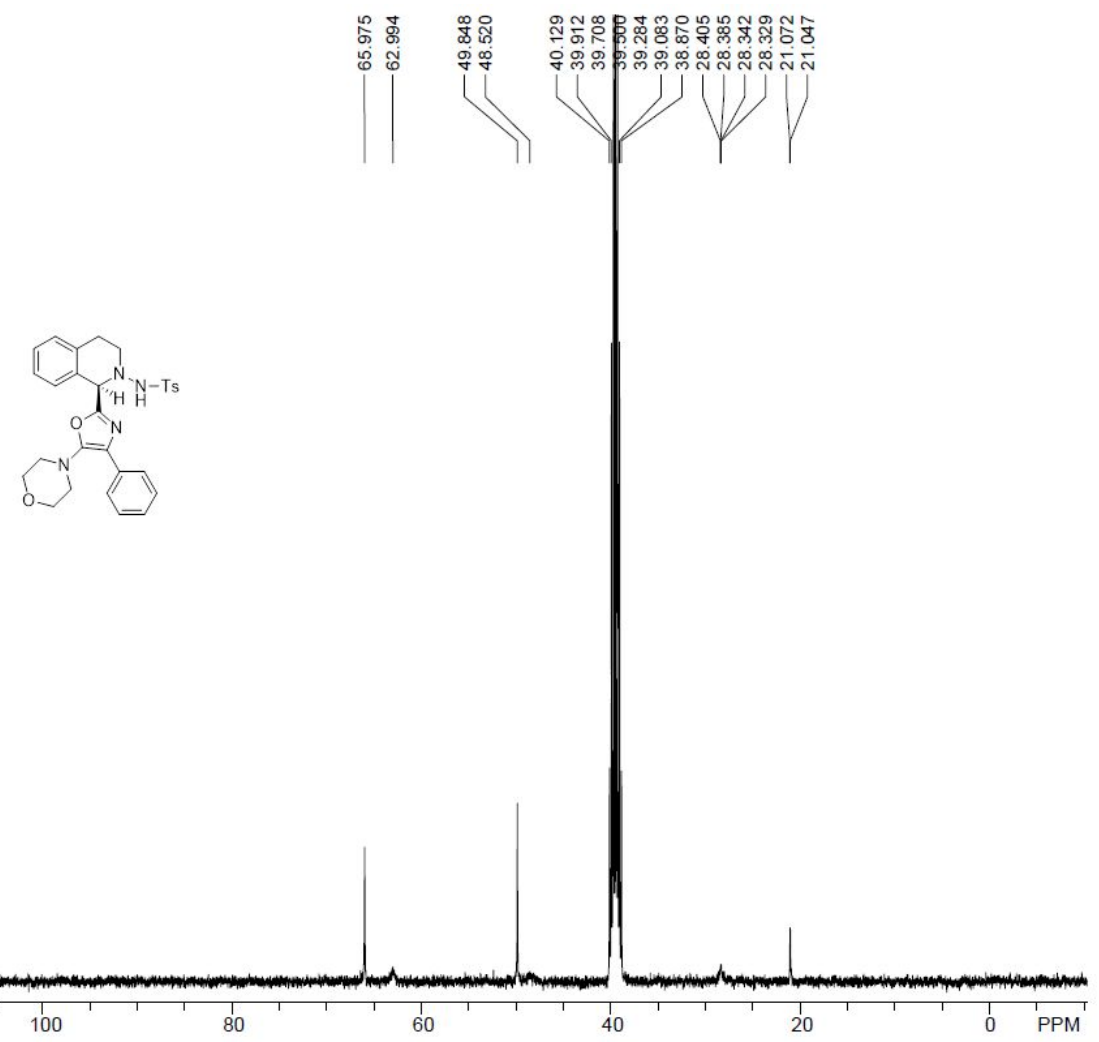

${ }^{1} \mathrm{H}$ NMR of $8 \mathbf{r}$
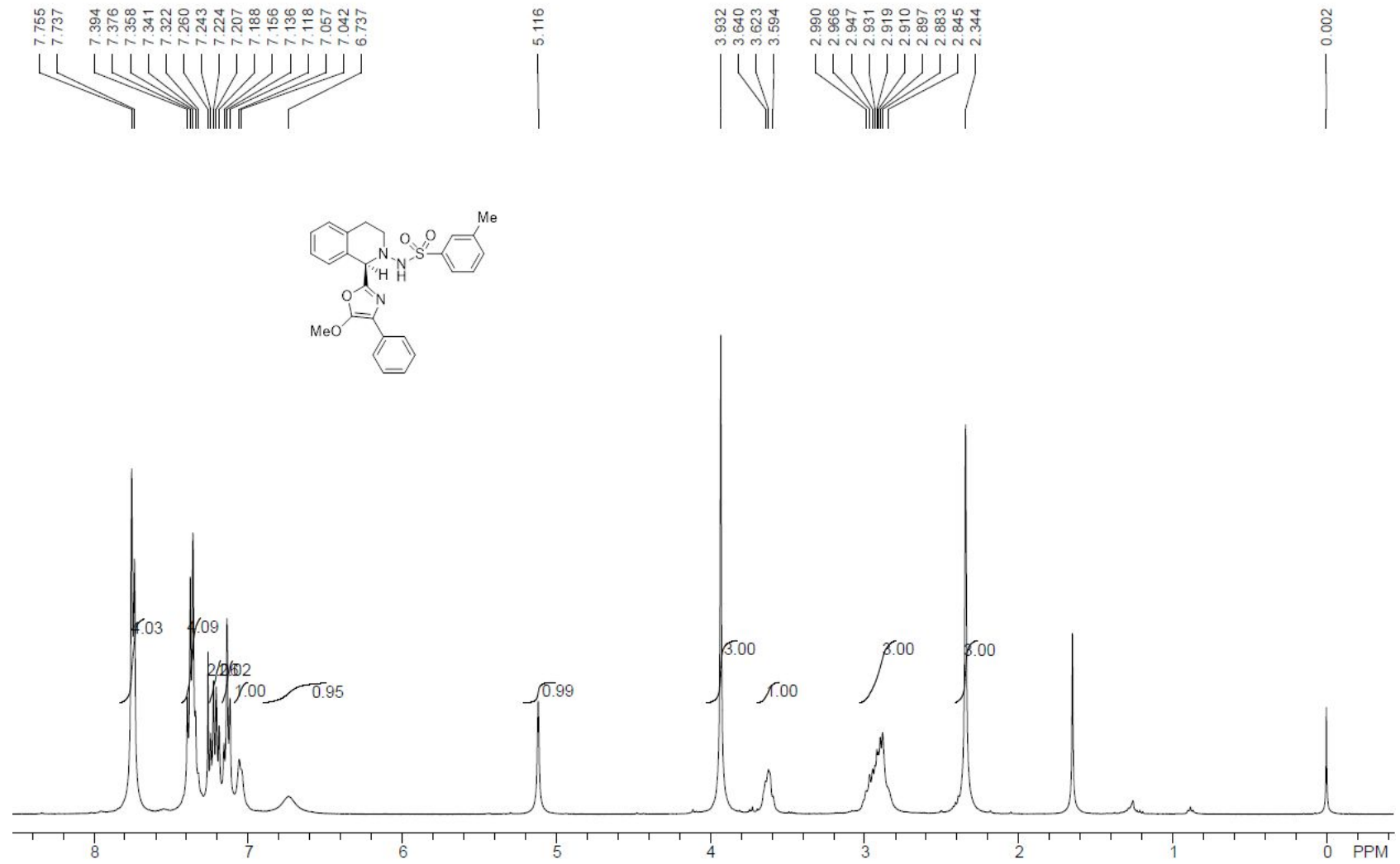


\section{${ }^{13} \mathrm{C}$ NMR of $\mathbf{8 r}$}
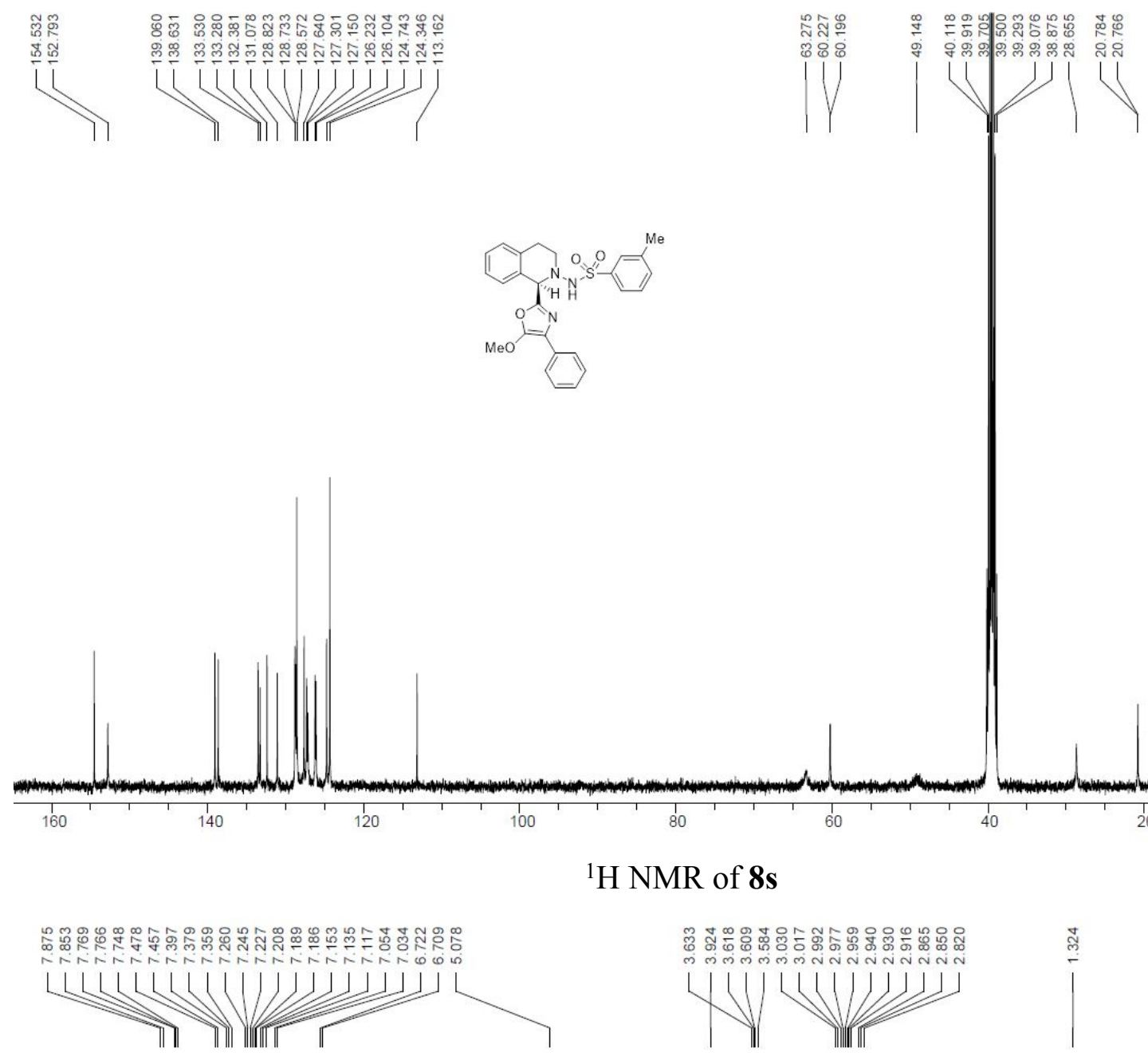

${ }^{1} \mathrm{H}$ NMR of $8 \mathbf{s}$
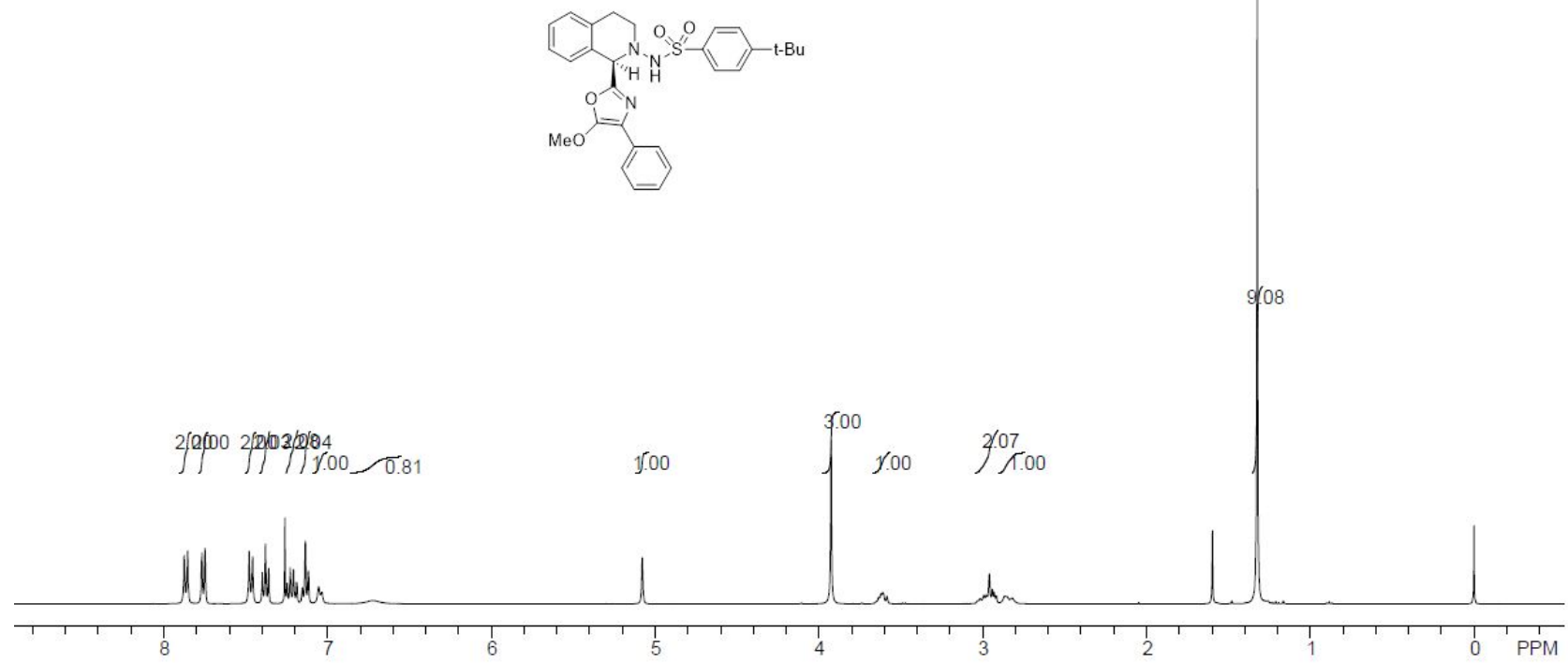
${ }^{13} \mathrm{C}$ NMR of $8 \mathrm{~s}$
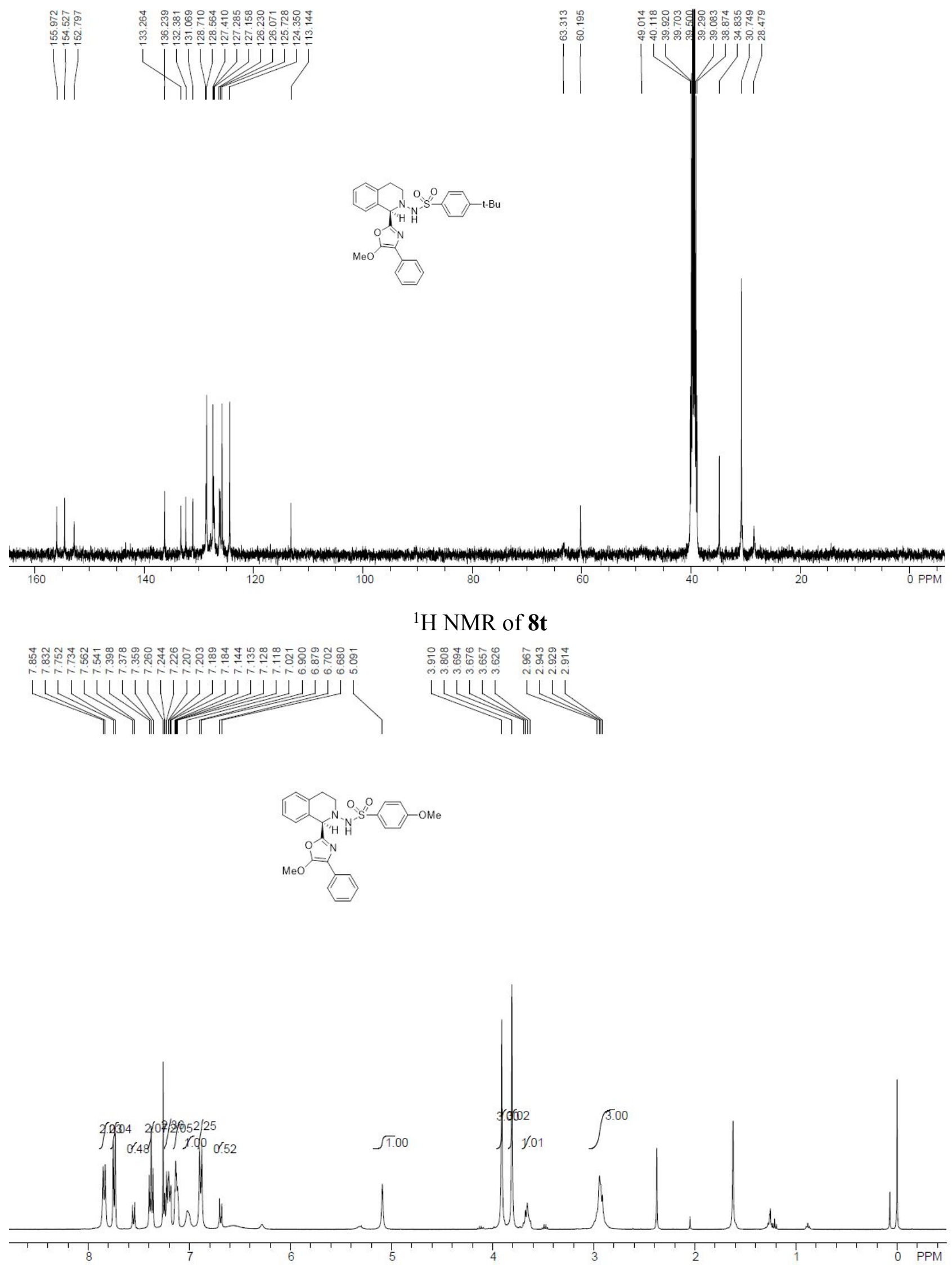
${ }^{13} \mathrm{C}$ NMR of $\mathbf{8 t}$
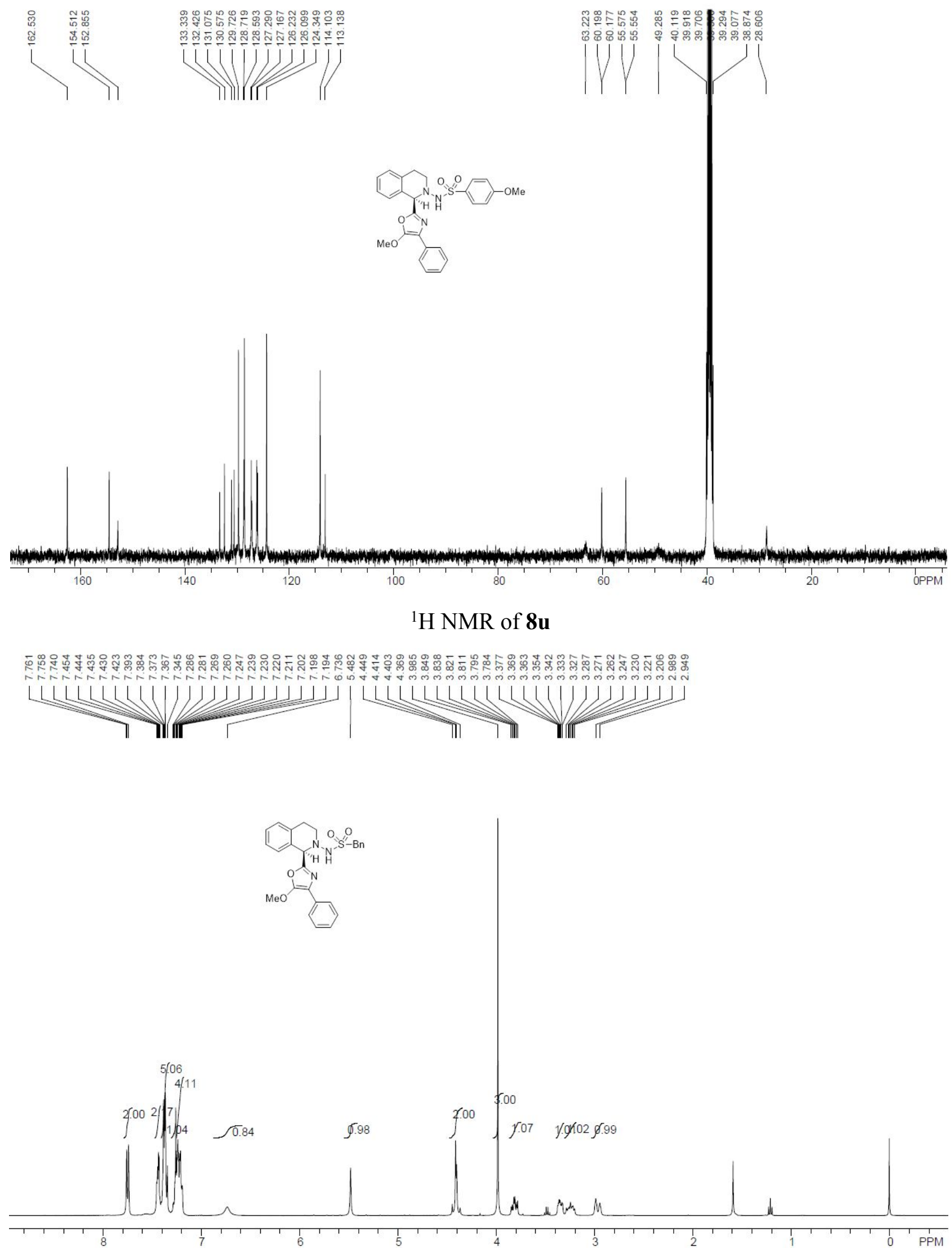
${ }^{13} \mathrm{C}$ NMR of $8 u$
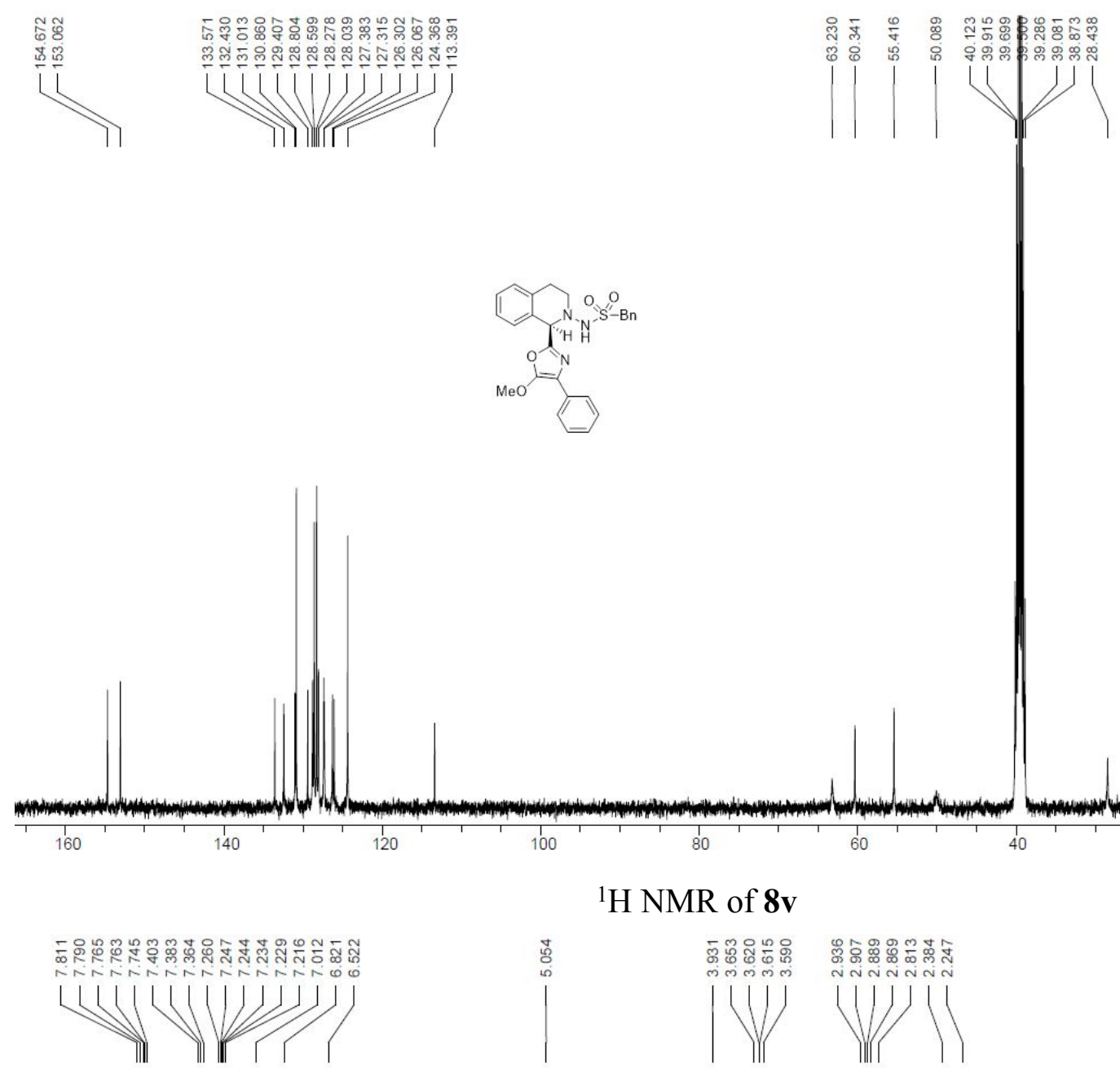

80
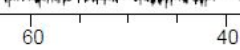

${ }^{1} \mathrm{H}$ NMR of $\mathbf{8 v}$

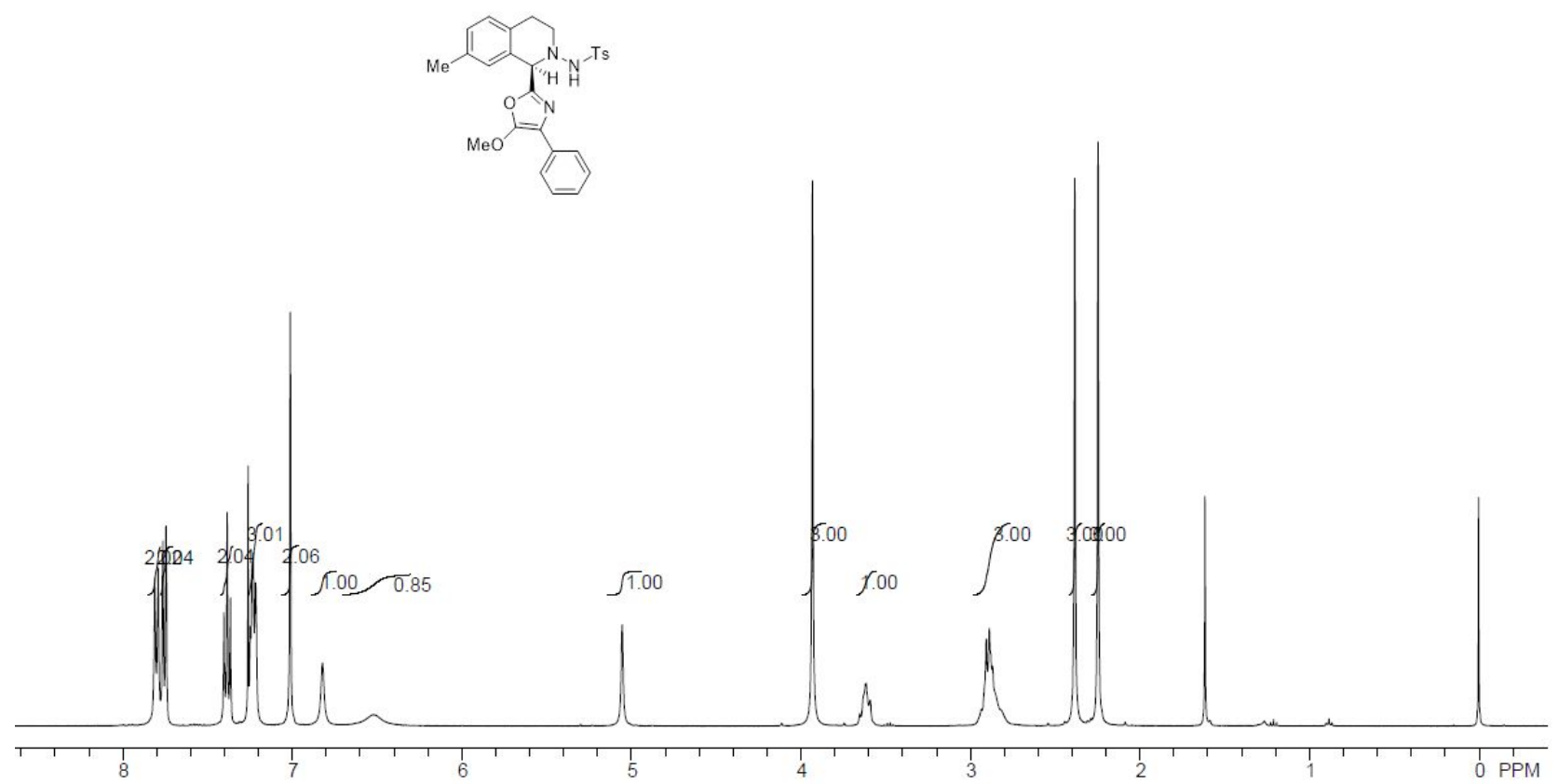


${ }^{13} \mathrm{C}$ NMR of $8 \mathbf{v}$

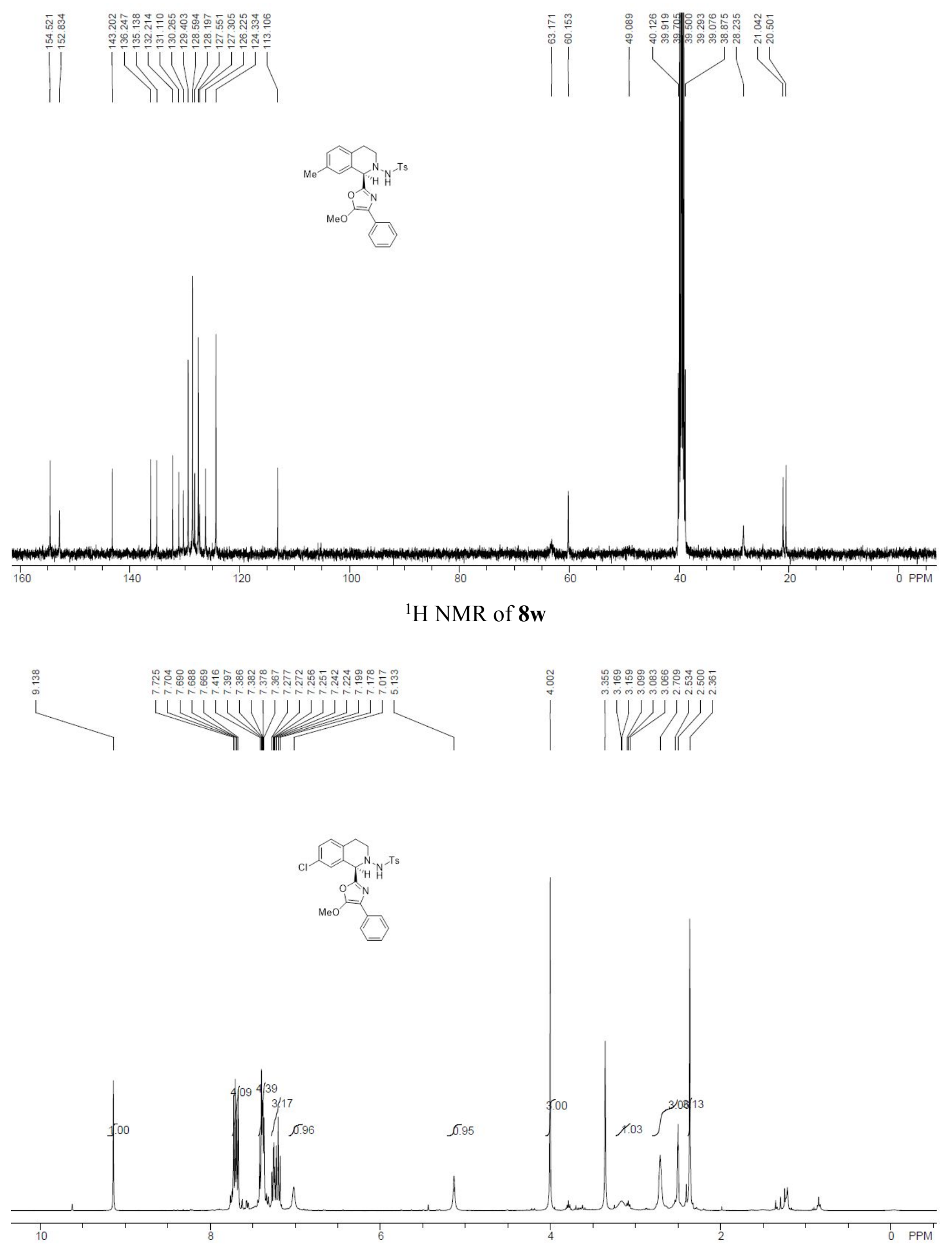


${ }^{13} \mathrm{C}$ NMR of $8 w$
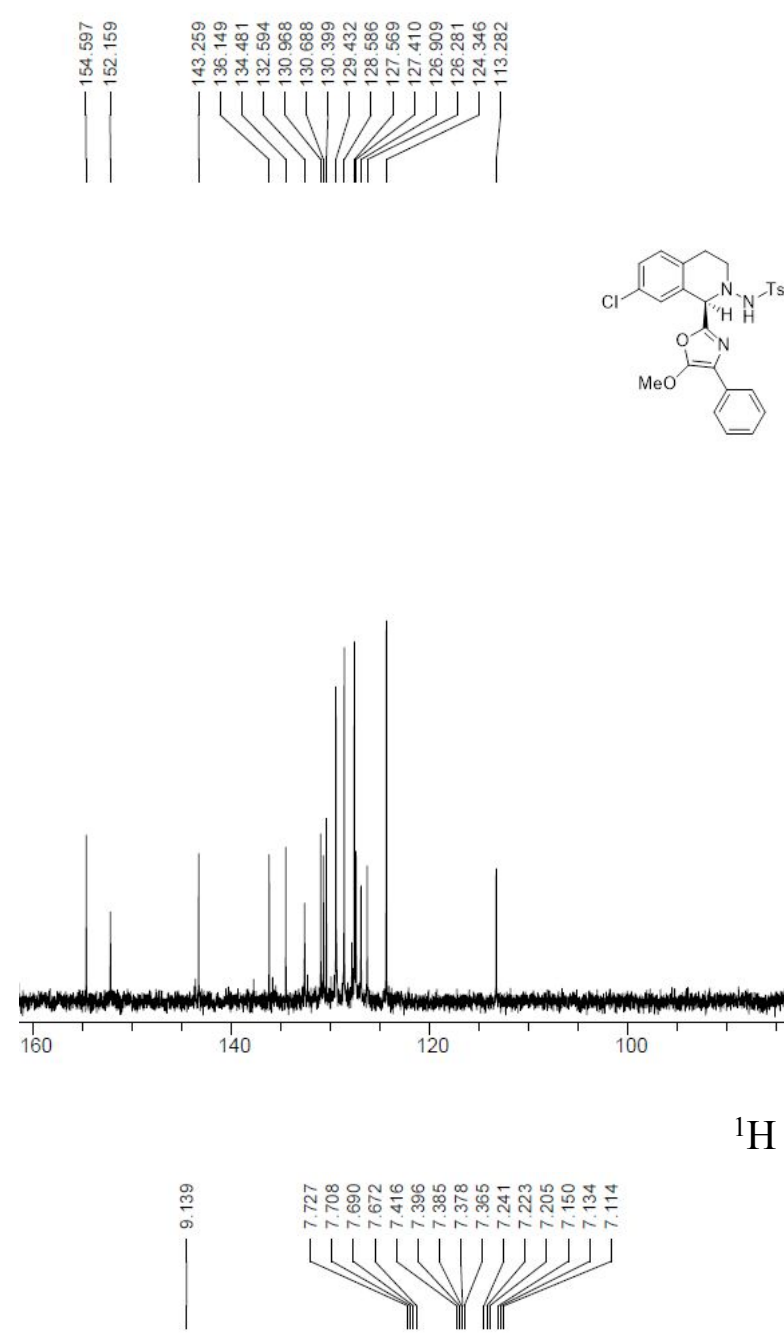

${ }^{1} \mathrm{H}$ NMR of $\mathbf{8 x}$

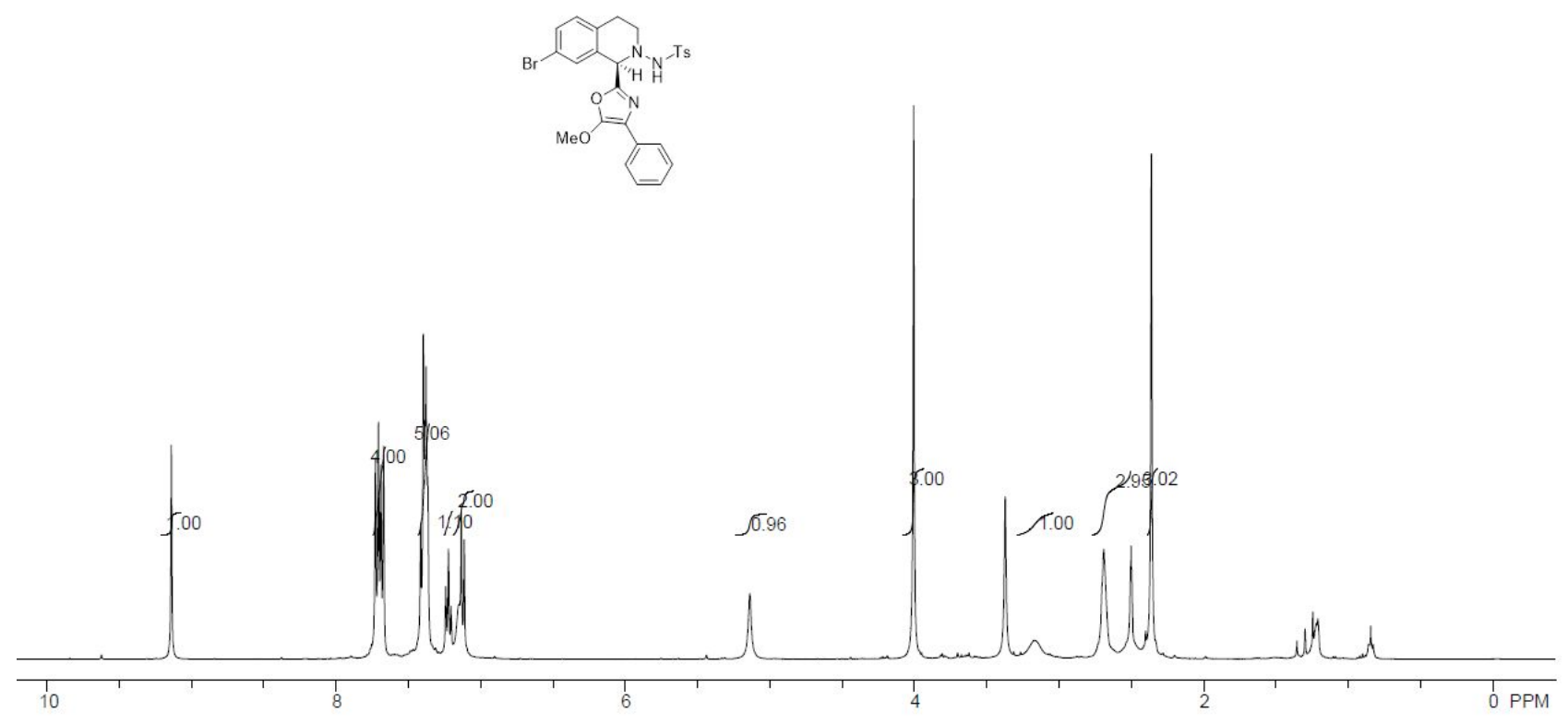


${ }^{13} \mathrm{C}$ NMR of $\mathbf{8 x}$
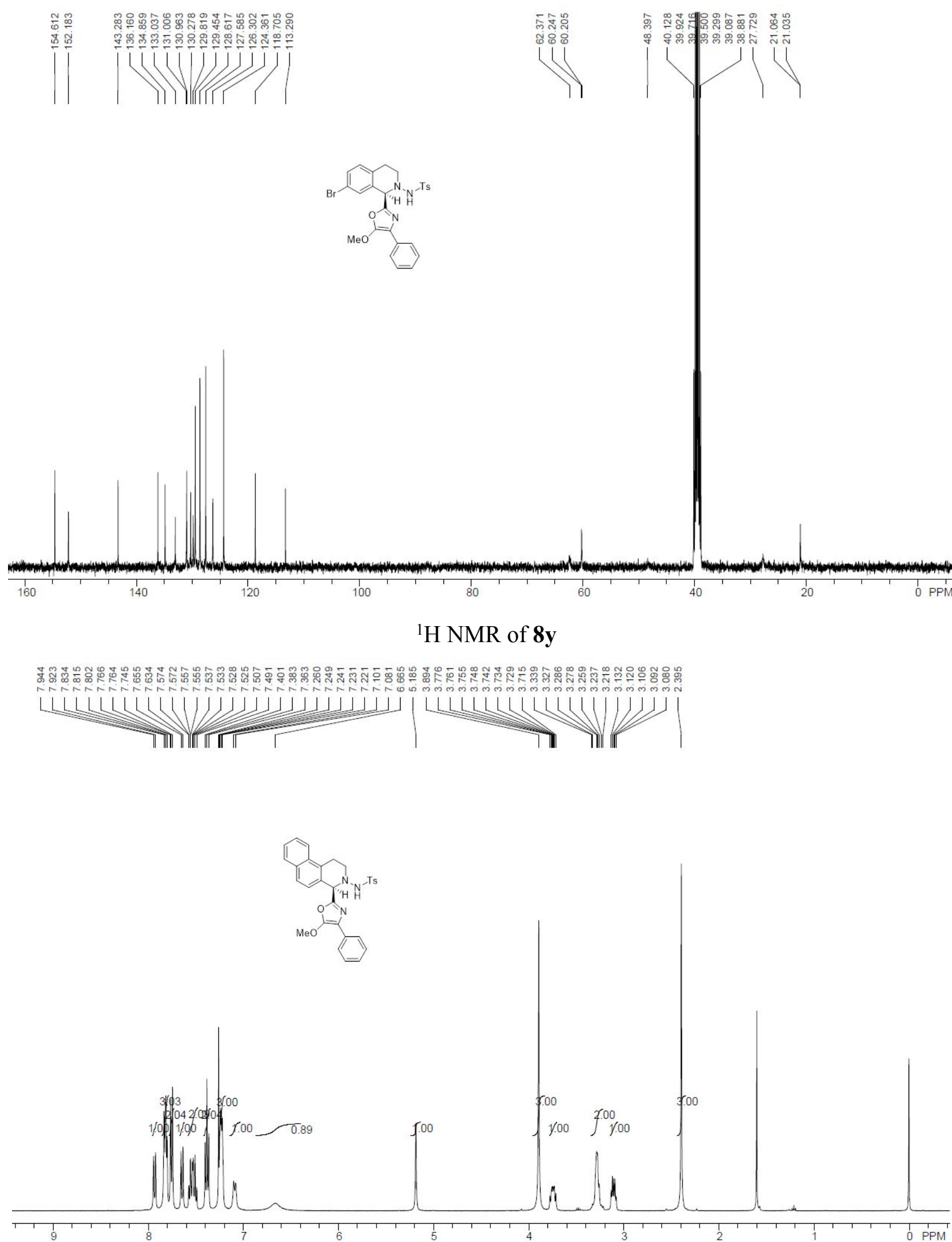
${ }^{13} \mathrm{C}$ NMR of $\mathbf{8 y}$
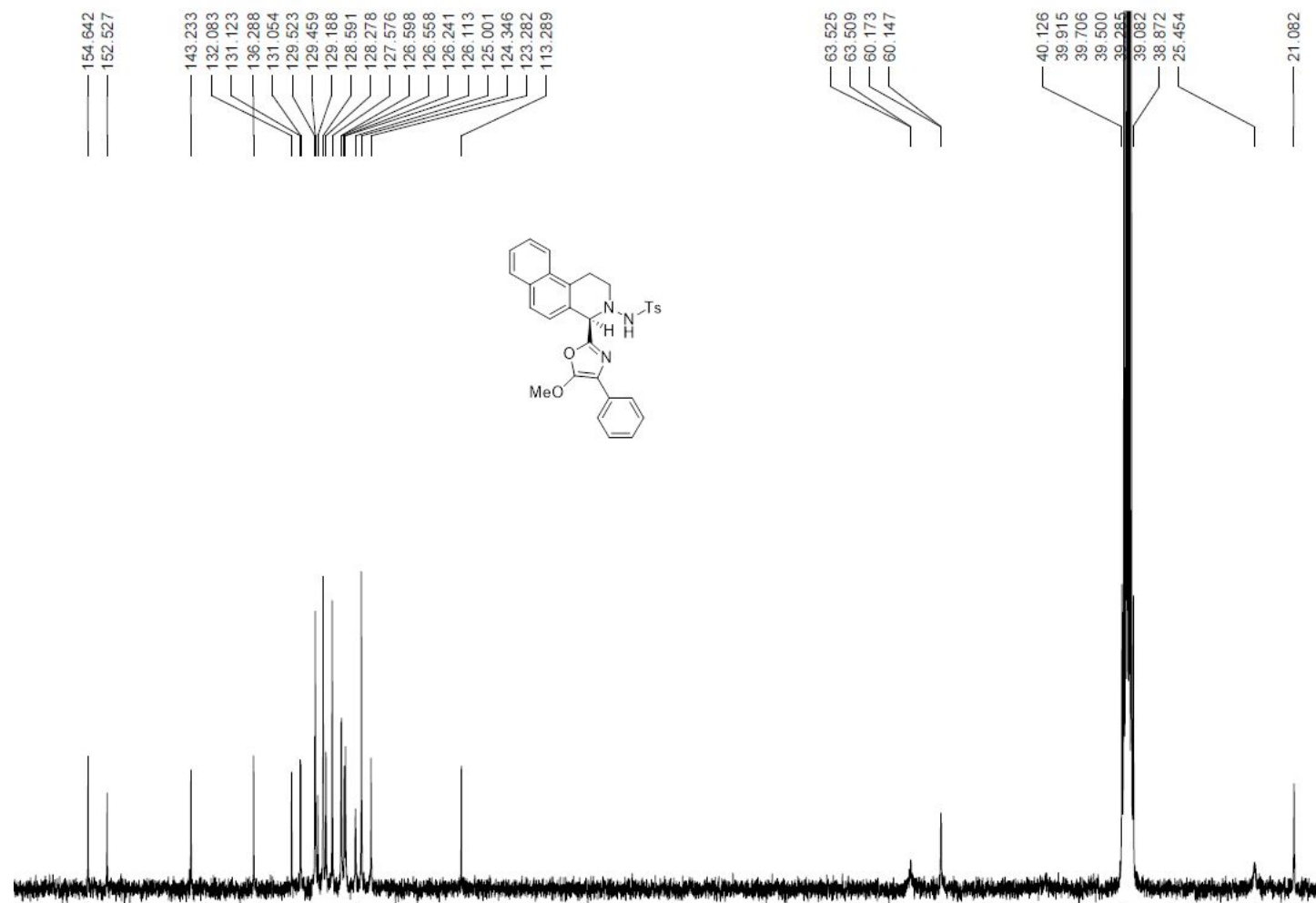